\title{
REPRESENTATIONS OF ALGEBRAS BY CONTINUOUS SECTIONS
}

\section{BY KARL HEINRICH HOFMANN}

ABSTRACT. This survey is concerned with the representation of discrete rings and topological algebras (such as $C^{*}$-algebras) by rings of continuous sections in sheaves of rings or fields of topological algebras.

\section{TABLE OF CONTENTS}

Preface and acknowledgments

\$1. Rings - the main algebraic example 296

Preliminaries

A general construction

The ring case

A second construction for rings $\quad 305$

The key theorem 307

Sheaves of local rings $\quad 308$

About minimal primes $\quad 314$

\$2. The Grothendieck construction for commutative rings and its relation to the constructions of $\$ 1$

Some background $\quad 316$

The Grothendieck sheaf and the key theorem 316

$\mathscr{R}^{\prime}$ and $\mathscr{G}$

§. Sectional representation over Boolean spaces regular and biregular rings

The Boolean decomposition principle

Pierce's Boolean decomposition theorem 324

Further applications of Boolean decomposition $\quad 326$

Baer rings

The patch topology on Spec $R \quad 328$

$\$ 4$. Sectional representation of lattice ordered structures 330

Generalities

Sheaves of l-rings and Keimel's main theorem 331

Local l-rings

An expanded version of an invited address delivered to the 679th meeting of the Society in Athens, Georgia on November 20,1970; received by the editors November 15, 1971 .

AMS 1970 subject classifications. Primary 06A70, 14A15, 16A10, 16A20, 16A66, 18F20, 46L05, 46L10, 46L 25, 55F65.

Key words and phrases. Sheaf, bundle, ring, $C^{*}$-algebra, $W^{*}$-algebra, regular ring, Baer ring, Boolean ring, harmonic ring, $f$-ring, $l$-ring, prime ideal, maximal ideal, local ring, spectrum of a ring, uniform field. 
Keimel's Boolean decomposition theorem 333

$\begin{array}{ll}\text { f-rings } & 334\end{array}$

§5. The foundations of the general theory of sectional representation 336 Uniform fields

The canonical field

Mulvey's adjunction

Localization

The localization theorem $\quad 352$

§7. Fields of topological rings and modules $\quad 354$

Fields of rings $\quad 354$

Partitions of identity $\quad 356$

$\begin{array}{ll}\text { Stone Weierstrass theorem } & 357\end{array}$

§8. Representations of topological rings by sections in fields 358

Localization for topological rings $\quad 359$

$C^{*}$-algebras $\quad 359$

Fields of Hilbert spaces $\quad 364$

Topological f-algebras $\quad 366$

Index

$\begin{array}{lr}\text { List of symbols } & 369\end{array}$

$\begin{array}{ll}\text { Bibliography } & 370\end{array}$

Preface and acknowledgements. This essay about some aspects of sectional representation began with an invited address at the 679th meeting of the American Mathematical Society in Athens, Georgia on November 20,1970 [44]. It has been greatly expanded and elaborated in order to provide a survey, at least to some extent, over the state of research in the area of sectional representation. A complete survey over this area is out of the question at this time; the theory has outgrown the stage in which all aspects of its development could have been equally and fairly discussed while keeping the length of the discourse reasonable. Thus this is a biased report, leaning somewhat towards the question "how to represent a (topological) ring of very general type by continuous sections in a canonical sheaf or field;" that is, the "one-ring-at-a-time" aspect is much more extensively treated than, say, the functorial aspect of sectional representation theory; and modules, while occurring here and there, stay somewhat in the background. Some of the material we present is new, and we organize some of the known material in novel form. Some of the content is based on earlier joint work with DAUNS; many of the more recent aspects of our presentation owe much not only to numerous discussions with Klaus Keimel, but also with Kwangil KoH and Silviu Teleman who visited Tulane during the academic year 1970-71. We refer to a volume of 
Lecture Notes in Mathematics ([54], [92]) which contains an account of the sectional representation theory of lattice ordered rings by KeIMEL and one of harmonic rings by TELEMAN; existence obviates the necessity of going into the details of these special theories in the appropriate places. In the compilation of the bibliography and pertinent material I had the kind assistance of numerous colleagues, notably G. M. BERGMAN, S. D. Comer, J. Dixmier and his student F. Perdrizet, B. R. Gelbaum, J. Kist, A. G. Mewborn, C. J. Mulvey, R. S. Pierce, S. A. Selesnick, R. WIEGAND, W. WILS; many of these provided me with material which had not appeared at the time and which hopefully will be available by the time this report appears. Again I have to mention KermeL in this context who indefatigably kept me up to the minute in new references which he discovered.

The work on this survey was supported by an NSF-grant as was some of my earlier work in the area. The visiting program at Tulane during 1970-71 which brought in КоH and KEIMEL and contributed partial support to Teleman was made possible through a grant by the FORD Foundation to Tulane University; the latter visited Tulane as a Fellow of the Exchange Program between the Academy of Sciences of the Socialist Republic of Rumania and the National Academy of Sciences of the United States.

Introduction. Sectional representation theory, in a nutshell, is concerned with the following idea: In order to describe the nature of some algebraic or topological structure $R$ (such as a ring or a topological group) one picks a family $f_{b}: R \rightarrow R_{b}, b \in B$, of surjective homomorphisms and, forming a disjoint union $E$ of the $R_{b}$, seeks to endow $E$ and $B$ with topologies such that

(i) the natural function $\pi: E \rightarrow B$, given by $\pi(x)=b$ iff $x \in R_{b}$, is continuous;

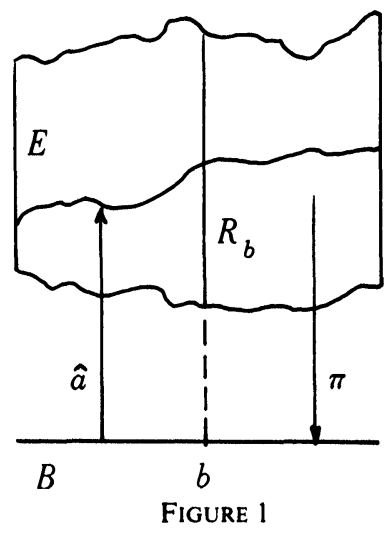


(ii) all functions $\hat{a}: B \rightarrow E$, for $a \in R$ given by $\hat{a}(b)=f_{b}(a)$ are continuous;

(iii) if $A$ and the $R_{b}$ have a topological structure, then the topology induced by $E$ on $R_{b}$ is the given one.

If $R$ and all $R_{b}$ are discrete, then (iii) is void.

All functions $\hat{a}$ satisfy the relation $\pi \hat{a}=1_{B}$ and each continuous function $\sigma: B \rightarrow E$ with $\pi \sigma=1_{B}$ is called a section (of $\pi$ ); the terms "right inverse" of $\pi$ or "coretraction" would be just as appropriate but have not been established in this context. The set $\Gamma(\pi)$ of all sections is a subset of the product $\prod\left\{R_{b}: b \in B\right\}$ and thus clearly inherits an algebraic structure if $R$ and $R_{b}$ have one; in general it will also be endowed with a topological or uniform structure, even though its selection may be a little less obvious because the structure induced from the product is generally not the one desirable for the practice. It is indeed a reasonable question to ask what one hopes to achieve with such a construction, if it should be possible at all.

The function $a \mapsto \hat{a}: R \rightarrow \Gamma(\pi)$ will be, in general, a homomorphism of the category of structures under consideration, and it is easily seen to be injective exactly if the family $\left\{f_{b}: b \in B\right\}$ of homomorphisms separates the points of $R$; this property may be considered as a sort of semisimplicity condition of $R$ (relative to the $f_{b}$ ). Historic reasons justify the choice of the name of Gelfand morphism for the map $a \mapsto \hat{a}$. If all the topologies in our brief outline are discrete, then $\Gamma(\pi)=\prod\left\{R_{b}: b \in B\right\}$, so the Gelfand morphism is just a subdirect representation of $R$ in the traditional sense and it now becomes plausible that the introduction of topology into the setup serves to sharpen the tool of subdirect representation. The deficiency of all subdirect representations, of course, is that the image $R$ of the Gelfand morphism in $\prod R_{b}$ is appallingly thin, and with the great discrepancy between $\hat{R}$ and $\prod R_{b}$ much of the gain is lost that was perhaps achieved by having found factors $R_{b}$ whose structure is much simpler than that of $R$. With the sectional representation approach we obtain $\hat{R} \subseteq \Gamma(\pi)$ and $\Gamma(\pi)$ may itself be much smaller than $\prod R_{b}$ : in particularly opportune circumstances (of which we will encounter a surprisingly large sample) we may even have $R=\Gamma(\pi)$ in which case the theory of sectional representations would have rendered a completely satisfactory service. A classical example which one might wish to keep in mind is the one of a commutative $C^{*}$-algebra $R$ with identity and with $B$ being the set of $C^{*}$-homomorphisms $f_{b}: R \rightarrow R_{b}=C$. We may then identify $E$ with $C \times B$ and $\pi$ with the projection on $B$, give $B$ the weak *-topology and observe that the $C^{*}$-algebra of all continuous functions $B \rightarrow C$ is isomorphic to $\Gamma(\pi)$ under the map $f \rightarrow(b \rightarrow(f(b), b))$. The Gelfand morphism is then the classical Gelfand representation, and the Gelfand Naimark theorem states that $\hat{R}=\Gamma(\pi)$ in our current interpretation.

We will demonstrate that the problem of sectional representation has a 
very general solution (5.20-21). That it is not, in my opinion, very satisfactory has the following reason: Firstly, under very general circumstances, the case $\hat{R}=\Gamma(\pi)$, or even anything approaching it, is rare. In the situation of rings, where $\hat{R}=\Gamma(\pi)$ occurs frequently for appropriate selections of morphisms $f_{b}$, one has inherent difficulties with those cases which are of most interest: the cases in which $B$ is a set of prime ideals and $f_{I}: R \rightarrow R / I$, $I \in B$, are just the quotient maps. The complications arise from the fact that we are given a topology on such a set, namely the hull-kernel topology. The canonical solution to the sectional representation problem as outlined above, however, will by necessity call for a topology on $B$ which with rare but noteworthy exceptions will be different from the hull-kernel topology. The hull-kernel topology has proved to be indispensable in so many instances in algebra and analysis, that one would be ill-advised to disregard its advantages. The solution, which in all instances, both in algebra and analysis, have provided satisfactory if not complete answers, is the modification of stalks by taking account of the given hull-kernel topology and constructing, in a suitable fashion, for each prime ideal $I$ in the given collection $B$, a possibly smaller ideal $\tilde{I}$ so that the new family of morphisms $\tilde{f}_{I}: R \rightarrow R / \tilde{I}, I \in B$, yields an entirely satisfactory sectional representation over $B$ with its hull-kernel topology.

In many respects, sectional representation is by no means a recent upstart. It is natural that forerunners in analysis antedate the earliest appearance of sectional representation in pure algebra; the existence of topologies with the properties we described is more likely to be noticed first in analytical contexts, and our example of the Gelfand representation of commutative Banach algebras is one of the first occurrences of sectional representation. The particular trend of thought to use sectional representation in the study of Banach algebras which started with commutative Banach algebras has continued up to very recent developments, as we shall see. A slightly different, although related type, of sectional representation, we may mention in passing is von Neumann's theory of measurable fields of Hilbert spaces, specifically designed for the reduction theory of operator algebras; it, too, precedes the genuine algebraic applications of the idea; we will, however, in this article not be concerned with measurable fields of Hilbert spaces. The ground for sectional representation in pure algebra was not ready until topological considerations became a legitimate part of algebra; in this particular instance the discovery of the now traditional topologies on spaces of prime ideals of rings by Jacobson, Stone, Zariski and others paved the way. The first sectional representation theorems were of the nature of the Gelfand Naimark theorem in analysis; that is, one obtained representations of certain rings or algebras as function rings or algebras. The classical example is the Stone representation theorem for 
Boolean algebras which was later generalized to certain biregular rings by Arens and Kaplansky. Gratifying as representations by function spaces are, they eventually appear as the trivial case of sectional representation theory in the sense that the space $E$ of our outline is just a product. It was then the arrival of the concept of a sheaf that in the algebraic context opened the way for the treatment of more general situations. In the foundations of algebraic geometry as laid by Grothendieck and Dieudonné this approach was widely used. The use of sheaves in the structural investigation of special classes of (not necessarily commutative) rings is more recent, in fact is not really much older than five years. The high degree of development of sheaf theory has certainly contributed to the flourishing of the algebraic portion of sectional representation theory. Yet in the meantime it has fanned out to an extent which makes it impossible at this time to claim completeness in a discussion like the one before us. The essentially new element in the theory, as we see it, is that one is now in a position to have unifying methods which allow us to treat the cases of interest to the analysts and topologists simultaneously with the algebraic version as special cases of one and the same theory. The sectional representation theory has only just passed its beginning stages, and numerous applications are to be expected. In particular, the topological version of the theory, which is by necessity the most general and more complicated than the plainly sheaf theoretic version, has a larger backlog to catch up and one may expect considerable progress not only in the foundations of the theory, but also, and in particular, in its applications.

While it will not be possible to cover all of the work in sectional representation theory in its relation to category theory, it must nevertheless be pointed out that even past the Grothendieck duality theorem one has now a large supply of adjunction theorems based on sectional representation due to the work of Mulvey. The first analytical version of such a theorem has only recently been established by Takahashi.

$\S 1$. Rings-the main algebraic example. In this section we illustrate certain essential features of sectional representation by discussing the representation of a discrete ring by continuous sections in a sheaf. More general methods will then be more profitably described in later sections.

Preliminaries. In the beginning we need a formal definition of a sheaf; we will therefore set out with two equivalent definitions of a sheaf of sets and a sheaf of abelian groups. The latter is the most frequently used concept ; from it one derives readily the ideas of a sheaf of rings (or, equivalently, a ringed space) which is the one needed for sectional representation of rings.

Definition 1.1 . We denote by $\mathscr{A}$ the category of sets or, respectively, the category of abelian groups or, more generally, the category of (left) 
$R$-modules for some ring $R$ with identity. Let $B$ be a topological space; we denote by $\mathcal{O}(B)$ the topology of $B$ which we consider as small category, whose objects are the open sets $U \subseteq B$ and whose morphisms are exactly the inclusion maps $U \rightarrow V$ with $U \subseteq V$. Then an $\mathscr{A}$-presheaf (i.e. a presheaf of sets, resp., abelian groups) is a functor $\Gamma: \mathcal{O}(B)^{\text {op }} \rightarrow \mathscr{A}$, where ? $?^{\mathrm{op}}$ denotes the forming of the opposite category (which is just a device to avoid speaking of contravariant functors). The space $B$ is called the base space. If the functor $\Gamma$ preserves limits of intersection-closed subdiagrams $\mathscr{U} \hookrightarrow \mathcal{O}(B)$ then it is called an $\mathscr{A}$-sheaf (i.e. a sheaf of sets, resp., abelian groups $R$ modules). The stalk $\Gamma_{b}$ of a presheaf (or sheaf) $\Gamma$ in $b \in B$ is defined by $\Gamma_{b}=$ colim $\Gamma D_{b}$ where $D_{b}: \mathscr{U}(b) \hookrightarrow \mathcal{O}(B)$ is the diagram of the filter of open neighborhoods of $b$ in $\mathcal{O}(B)$.

We remark that in $\mathcal{O}(B)$, as in any partially ordered set considered as a category, the colimit of a diagram is the l.u.b. of the objects in the diagram; thus in $\mathcal{O}(B)^{\mathrm{op}}$ the limit of a diagram is exactly the union of the collection of open sets in the diagram with the natural inclusion maps (reversed) as limit maps. If for an open set $U \subseteq B$ one interprets $\Gamma(U)$ as the set of local sections over $U$ given by the presheaf $\Gamma$, then the continuity condition for the functor $\Gamma$ means that a matching collection of local sections over some family of open sets may be patched to a unique section over the union of these open sets.

There is an alternative, geometric description of a sheaf which for the purposes of sectional representation is frequently more useful (in particular, since it leads to generalizations of the kind that are needed in functional analysis). The definition above is more general in that it may be phrased for any category $\mathscr{A}$ whatsoever; the actual construction of sheaves in general categories, however, may be very delicate if the direct limit is more complicated than for sets or abelian groups.

Definition 1.2. Let $\pi: E \rightarrow B$ be a surjective function which is a local homeomorphism (which means that for each $x \in E$ there are open sets $U$ and $V$ in $E$ and $B$, respectively, such that $x \in U$ and $\pi \mid U: U \rightarrow V$ is a homeomorphism). Then $\pi$ is called a sheaf of sets (or a sheaf projection) and $E_{b}=\pi^{-1}(b)$ is called the stalk of the sheaf in $b$. If all stalks are abelian groups and if the function $(x, y) \mapsto x-y: E \times{ }_{\pi} E \rightarrow E$ (with $E \times \times_{\pi} E$ $=$ pullback of $\{\pi, \pi\}=\{(x, y) \in E \times E \mid \pi(x)=\pi(y)\})$ is continuous, then $\pi$ is called a sheaf of abelian groups. The space $E$ is called the sheaf space. If every $E_{b}$ is a ring with identity $1(b)$ (resp., a left $R$-module), then $\pi$ is a sheaf of rings with identity (left $R$-modules) if also $(x, y) \mapsto x y: E \times_{\pi} E \rightarrow E$ and $b \mapsto 1(b): B \rightarrow E$ (resp. $(r, x) \mapsto r \cdot x: R \times E \rightarrow E$ ) are continuous. A sheaf of rings is also called a ringed space.

Construction 1.3. If $\pi: E \rightarrow B$ is a sheaf (of sets), then one defines, for each open set $U \subseteq B$, the set $\Gamma(\pi, U)$ to be the collection of all continuous func- 
tions $\sigma: U \rightarrow E$ with $\pi \sigma=1_{U}$; these functions are called sections over $U$. Then, via restriction, $\Gamma(\pi, ?): \mathcal{O}(B)^{\text {op }} \rightarrow$ Set is a functor which is rather directly shown to be a sheaf in the sense of 1.1. Conversely, if $\Gamma: \mathcal{O}(B)^{\text {op }}$ $\rightarrow$ Set is a presheaf of sets, we let $E$ be the disjoint union of the family of stalks $\Gamma_{b}, b \in B$. If $b \in U \in \mathcal{O}(B)$, let $\lambda_{b, U}: \Gamma(U) \rightarrow \Gamma_{b}$ be the colimit natural transformation; then the collection of all $\left\{\lambda_{b, U}(\sigma) \mid b \in U\right\}, \sigma \in \Gamma(U), U \in \mathcal{O}(B)$, is a basis for a topology on $E$, relative to which the obvious map $\pi: E \rightarrow B$ is a local homeomorphism and so defines a sheaf in the sense of 1.2. This construction carries over to the case of abelian groups, rings with identity, left $R$-modules. There is a natural transformation $\gamma: \Gamma \rightarrow \Gamma(\pi, ?)$, given by $\gamma_{U}(\sigma)(b)=\lambda_{b, U}(\sigma)$ for $\sigma \in \Gamma(U)$; moreover $\gamma$ is an isomorphism iff $\Gamma$ is a sheaf.

A general construction. We now describe a typical construction of a sheaf as it arises in sectional representation.

Construction 1.4. Let $B$ be an arbitrary topological space and $A$ an abelian group. Suppose that there is a family $\left\{K_{b} \mid b \in B\right\}$ of subgroups of $A$ indexed by $B$. For $U \in \mathcal{O}(B)$ define $\Gamma(U)=A / K(U)$ with $K(U)=\bigcap\left\{K_{b} b \in U\right\}$. If $V \subseteq U$ in $\mathcal{O}(B)$ then $U \rightarrow V$ in $\mathcal{O}(B)^{\text {op }}$ and $K(U) \subseteq K(V)$. Thus there is a natural morphism of abelian groups $\Gamma(U) \rightarrow \Gamma(V)$ and $\Gamma: \mathcal{O}(B)^{\text {op }} \rightarrow \mathrm{Ab}$ (where $\mathrm{Ab}$ denotes the category of abelian groups) is a presheaf. We compute the stalks of this presheaf. For each $U \in \mathcal{O}(B)$ we have an exact sequence

$$
0 \rightarrow K(U) \rightarrow A \rightarrow \Gamma(U) \rightarrow 0 .
$$

The stalk $\Gamma_{b}$ is colim $\Gamma(U)$ where $U$ ranges through the filter $\mathscr{U}(b)$ of open neighborhoods of $b$. This colimit is a direct limit and the direct limit functor preserves exactness in $\mathrm{Ab}$. Hence, if $\widetilde{K}_{b}$ denotes colim $K(U), U \in \mathscr{U}(b)$, then

$$
0 \rightarrow \widetilde{K}_{b} \rightarrow A \rightarrow \Gamma_{b} \rightarrow 0
$$

is exact.

We may, therefore, identify $\Gamma_{b}$ with $A / \widetilde{K}_{b}$. Now $K: \mathcal{O}(B)^{\mathrm{op}} \rightarrow \mathrm{Ab}$ is a functor mapping all morphisms into inclusion maps between subgroups of $A$. A colimit over a directed system is, therefore, just the set theoretic union of subgroups of $A$. Thus

$$
\tilde{K}_{b}=\bigcup_{b \in U} K(U)=\bigcup_{b \in U} \bigcap\left\{K_{c} \mid c \in U\right\} .
$$

The sheaf space $E$ of the associated sheaf is consequently the disjoint union of the groups $A / \widetilde{K}_{b}$; in order to guarantee disjointness we write $E_{b}=A / \widetilde{K}_{b}$ $\times\{b\}$. The topology on $E$ is generated by the basic sets $\tilde{a}(U), a \in A$, $U \in \mathcal{O}(B)$, where $\tilde{a}: B \rightarrow E$ for an $a \in A$ is the section defined by $\tilde{a}(b)=$ $\left(a+\widetilde{K}_{b}, b\right) \in A / \widetilde{K}_{b} \times\{b\}=E_{b}$. The sheaf projection $\pi: E \rightarrow B$ is given by 
$\pi\left(a+\widetilde{K}_{b}, b\right)=b$. We define $\tilde{K}(U)=\bigcap\left\{\widetilde{K}_{b} \mid b \in U\right\}$ for an open set $U \in \mathcal{O}(B)$, and observe

LEMMA 1.5. $\widetilde{K}(U)=K(U)$ for each $U \in \mathcal{O}(B)$, i.e. as functors $\mathcal{O}(B)^{\mathrm{op}} \rightarrow \mathrm{Ab}$, we have $\widetilde{K}=K$.

Proof. The inclusion $\tilde{K}(U) \cong K(U)$ follows trivially from $\tilde{K}_{b} \subseteq K_{b}$. Conversely, let $a \in K(U)$. Then, by the definition of $\widetilde{K}(U)$, we have $a \in \widetilde{K}_{b}$ for all $b \in U$. Hence $a \in \widetilde{K}(U)$.

As a consequence of Lemma 1.5 we have $\Gamma(U)=A / \widetilde{K}(U)$.

An element $s \in \Gamma(U)$ for a presheaf $\Gamma$ is called locally zero if for each $b \in U$ there is an open set $V$ with $b \in V \subseteq U$ such that the image of $s$ in $\Gamma(V)$ is zero. (Equivalently, $s$ is locally zero if its image is zero in every stalk $\Gamma_{b}$ with $b \in U$.) With this concept we can rephrase Lemma 1.5 as follows: Every locally zero section over $U$ is zero; or, once more in an equivalent fashion:

LEMMA 1.6. The natural transformation $\gamma: \Gamma \rightarrow \Gamma(\pi, ?)$ into the associated sheaf is an injection.

Since $\Gamma(B)=A / K(B)$ we have

LEMMA 1.7. The natural morphism of abelian groups $a \mapsto \tilde{a}: A \rightarrow \Gamma(\pi, B)$ has the kernel $\bigcap\left\{K_{b} \mid b \in B\right\}$.

The morphism of Lemma 1.7 is occasionally called the Gelfand morphism. Because of the significance of 1.5 and 1.6, we present the same result in a geometric version. Let $\pi: E \rightarrow B$ be the sheaf projection and call the subspace $\left\{\left(a+\widetilde{K}_{b}, b\right) \in E_{b} \mid a \in K_{b}, b \in B\right\}$ of $E$ the radical $\operatorname{Rad} E$ of $E$. (Note that the radical is not associated with any sheaf of abelian groups, but only with sheaves constructed as in 1.4.)

Then we can reformulate 1.5 as

Lemma 1.8. The interior of the radical $\operatorname{Rad} E$ is the zero section $O(B)$.

Proof. If $x=\left(a+\widetilde{K}_{b}, b\right) \in E_{b}$ is in the interior of $\operatorname{Rad} E$, then there is an open neighborhood $U$ of $b$ and a section $\sigma: U \rightarrow \operatorname{Rad} E$ in $\Gamma(\pi, U)$, with $\sigma(b)=\left(a+\widetilde{K}_{b}, b\right)$. Since $\pi$ is a sheaf the sections $\sigma$ and $\tilde{a}$ agree on an open neighborhood $V$ of $b$. Thus, by the definition of the radical $\operatorname{Rad} E$, we have $\sigma(c)=\left(a+K_{c}, c\right) \in K_{c} / \widetilde{K}_{c} \times\{c\}$ for all $c \in V$, i.e. $a \in \bigcap\left\{K_{c} \mid c \in V\right\}=K(V)$ $=\widetilde{K}(V)$ by 1.5 ; in particular, $a \in \widetilde{K}_{b}$, and thus $x=0(B)$.

In general, $\Gamma$ will not be a sheaf, i.e. $\Gamma(\pi, U)$ will be larger than $\Gamma(U)$ $=A / K(U)$. However, for the purposes of sectional representation one is frequently satisfied with information about the special case $U=B$ : Namely, one would like to answer the question

(Q) When is $\Gamma(B)=\Gamma(\pi, B)$, i.e. when is the natural morphism $a \mapsto \tilde{a}: A$ $\rightarrow \Gamma(\pi, B)$ surjective? 
For arbitrary spaces $B$ and arbitrary families $K_{b}$ one cannot hope for any reasonable answer to this problem. However, in the case of rings and modules answers are available which may be considered largely satisfactory.

The ring case. Our setting is the following: Let $R$ be a ring (momentarily not necessarily having an identity), let $B$ be a space of proper prime ideals with the hull-kernel topology. (Recall that on the space $\operatorname{Spec} R$ of all prime ideals the hull-kernel topology has a subbasis of sets

$$
S(a)=\{I \in \operatorname{Spec} R \mid a \notin I\}, \quad a \in A ;
$$

and a set $X \cong \operatorname{Spec} R$ is closed iff $X=\{I \in \operatorname{Spec} R \mid \bigcap X \subseteq I\}$.) We call a space quasi-compact if it satisfies the Heine Borel property. Let us record

Lemma 1.9. A closed subspace $X$ of $B$ is quasi-compact if $X$ satisfies the following conditions:

(X) If $I \subset R$ is a proper ideal with $\bigcap X \cong I$ then $I \subseteq J$ for some $J \in X$.

(e) $R=(e)+\bigcap X$ for some $e \in R$.

(IDEA OF THE PROOF. For any family $\mathscr{F}$ of closed sets of $X$ the conditions $I \in \bigcap \mathscr{F}$ and $\sum\{\bigcap F \mid F \in \mathscr{F}\} \subseteq I$ are equivalent. Indeed the latter means $\bigcap F \cong I$ and thus $I \in \bar{F}=F$ for all $F \in \mathscr{F}$. By hypothesis $(\mathrm{X})$, we thus have $\bigcap \mathscr{F}=\varnothing$ iff $\sum\{\bigcap F \mid F \in \mathscr{F}\}=R$. The latter implies $e \in \sum\{\bigcap F \mid F \in \mathscr{F}\}$, hence $e=r_{1}+\cdots+r_{n}$ for suitable $r_{k} \in \bigcap F_{k}, F_{k} \in \mathscr{F}$. This means $R=(e)$ $+\bigcap X \cong \bigcap F_{1}+\cdots+\bigcap F_{n}$ which, by $(\mathrm{X})$, is equivalent to $F_{1} \bigcap \ldots \bigcap F_{n}$ $=\varnothing$. Thus if $\mathscr{F}$ is a filterbasis of closed sets on $X$ then $\bigcap \mathscr{F} \neq \varnothing$.)

It will be essential to have the following technique which is known as partition of identity:

Lemma 1.10. Let $X$ be a subspace of $B$ and $e \in R$. If $X$ satisfies $(X)$ above then for any finite open cover $\bar{X} \cong U_{1} \cup \cdots \cup U_{n}$ of $B$ there are elements $r_{k} \in \bigcap\left(B \backslash U_{k}\right), k=1, \ldots, n$, with $e \equiv r_{1}+\cdots+r_{n} \bmod \bigcap X$. In particular, if $1 \in R$, this applies to $X=B$ and $e=1$.

(The proof is standard and similar to the one for 1.9: Since $\bar{X}$ $\cap \bigcap_{k=1}^{n}\left(B \backslash U_{k}\right)=\varnothing$ we have $\bigcap X+\bigcap\left(B \backslash U_{1}\right)+\cdots+\bigcap\left(B \backslash U_{n}\right)=R$ by $(\mathrm{X})$.)

We now approach the objective of representing a left $R$-module $M$ by a module of sections. Let $I \in B$ and let $I \cdot M$ be the submodule generated by all $i \cdot m$ with $i \in I$ and $m \in M$. Let $K_{I}$ be any submodule of $M$ with $I \cdot M$ $\subseteq K_{I}$. We apply Construction 1.4 to $B$ and the family $\left\{K_{I} \mid I \in B\right\}$ and obtain a presheaf $\Gamma: \mathcal{O}(B)^{\mathrm{op}} \rightarrow \mathrm{Ab}^{R}$ of left $R$-modules. Let $\mu: E \rightarrow B$ be the associated sheaf, $X \subseteq B$ a quasi-compact closed subspace and $\sigma: X \rightarrow E$ a continuous section over $X$. Recall $E_{I} \cong M / \tilde{K}_{I}$, say $E_{I}=M / \tilde{K}_{I} \times\{I\}$. For each $I \in X$ there is an element $m_{I} \in M$ with $\sigma(I)=\left(m_{I}+K_{I}, I\right) \in E_{I}$. The two sections $\sigma$ and $\tilde{m}_{I} \mid X$ of the sheaf $\mu \mid \mu^{-1}(X): \mu^{-1}(X) \rightarrow X$ agree in $I$, hence on a whole neighborhood of $I$ in $X$. Using the quasi-compactness of 
$X$ we find open sets $U_{1}, \ldots, U_{n}$ in $B$ whose union covers $X$, and we find elements $m_{1}, \ldots, m_{n}$ so that $\sigma\left|\left(X \cap U_{k}\right)=\tilde{m}_{k}\right|\left(X \cap U_{k}\right)$. Using the $m_{k}\left(U_{k}\right)$ and 1,2 one obtains also an extension of $\sigma$ to a section $\sigma$ over an open neighborhood of $X$. At this point we make the following assumption about $X$ :

(a) Every proper ideal of $R$ containing $\bigcap X$ is contained in $(\mathrm{X}, \mathrm{e})$ some ideal of $X$, and

(b) there is a relative identity $e$ modulo $\bigcap X$ in $R$ so that $e \cdot m$ $-m \in I \cdot M$ for all $m \in M$ and $I \in X$.

Note that $(\mathrm{X}, \mathrm{e})$ is satisfied if $1 \in R$ (with $e=1$ ) and if $X$ contains all maximal ideals of $R$ which contain $\bigcap X$. According to 1.10 we select elements $r_{0}, \ldots, r_{n}$ with $r_{k} \in \bigcap\left(B \backslash U_{k}\right)$ for $k=1, \ldots, n$ and with $r_{0}=e-\left(r_{1}+\right.$ $\left.\cdots+r_{n}\right) \in \bigcap X$. We set $m=r_{1} \cdot m_{1}+\cdots+r_{n} \cdot m_{n}$. We now claim $\tilde{m} \mid X$ $=\sigma:$ We have to show that $\sigma(I)=\tilde{m}(I)=\left(m+\tilde{K}_{I}, I\right) \in E_{I}$ for all $I \in X$ : If $I \in U_{k}$, then $r_{k} \cdot\left(\sigma-\tilde{m}_{k}\right)(I)=0$, since $\sigma$ and $\tilde{m}_{k}$ agree on $U_{k}$. If $I \notin U_{k}$, then $r_{k} \in \bigcap\left(B \backslash U_{k}\right) \subseteq I$ and thus $r_{k} \cdot\left(\sigma-\tilde{m}_{k}\right)(I) \in I \cdot E_{I} \cong I \cdot\left(M / \tilde{R}_{I}\right)$ $\subseteq I \cdot M / \tilde{K}_{I} \subseteq K_{I} / \tilde{K}_{I}$; thus $r_{k} \cdot\left(\sigma-\tilde{m}_{k}\right)(X) \subseteq \operatorname{Rad} E \cap \mu^{-1}(X)$ (see 1.8 for $\operatorname{Rad} E)$. Hence $\sum_{k=1}^{n} r_{k} \cdot\left(\sigma-\tilde{m}_{k}\right)(X) \subseteq \operatorname{Rad} E \cap \mu^{-1}(X)$, whence $\left(e-r_{0}\right) \cdot \sigma-\tilde{m} \mid X=\sum_{k=1}^{n} r_{k} \cdot\left(\sigma-\tilde{m}_{k} \mid X\right)=0$ by 1.8 ; since $r_{0} \in \bigcap X \in I$ for all $I \in X$, in a similar fashion we have $r_{0} \cdot \sigma=0$. Finally, $\sigma(I)-e \cdot \sigma(I)$ $\in I \cdot E_{I}$ for all $I \in X$ by $(\mathrm{X}, \mathrm{e})(\mathrm{b})$. Again as before, using that the interior of $\operatorname{Rad} E$ is $O(B)$, we conclude $\sigma=e \cdot \sigma$. Therefore $\sigma-\tilde{m} \mid X=(\sigma-e \cdot \sigma)$ $+r_{0} \cdot \sigma+\left[\left(e-r_{0}\right) \cdot \sigma-\tilde{m} \mid X\right]=0$.

REMARK 1 . We have in fact shown that every continuous section over a closed quasi-compact set $X \subseteq B$ with (X, e) extends to a global section in the sheaf $\mu$.

REMARK 2. Since the $S(a), a \in R$ form a basis of $\mathcal{O}(B)$ we could have assumed $U_{k}=S\left(a_{k}\right), a_{k} \in R$.

REMARK 3. If $\sigma$ is a global section with support $X$ and if $m$ is constructed for $\sigma \mid X$ as in the preceding process, then $\sigma=\tilde{m}$ : We may assume that $\tilde{m}\left|U_{k}=\sigma\right| U_{k}$, and the previous argument shows $\sum_{1}^{m} p_{k} \cdot\left(\sigma-\tilde{m}_{k}\right)=0$; but $(\sigma-e \cdot \sigma)+r_{0} \cdot \sigma$ vanishes on $B \backslash X$.

Let us note that relative identities have the following properties:

(i) If $e$ is a relative identity modulo $I$ and $I \cong J$, then $e$ is a relative identity modulo $J$.

(ii) If $e$ and $f$ are relative identities modulo $I$, respectively, $J$, then $e+f-e f$ is a relative identity modulo $I \leqq J$.

Thus, if $Y \subseteq X \leqq B$, then (X, e) implies ( $\mathrm{Y}, \mathrm{e})$; further $(\mathrm{X}, \mathrm{e})$ and $(\mathrm{Z}, \mathrm{f})$ imply $(X \cup Z, e+f-e f)$.

Definition 1.11. (i) A collection $\Phi$ of closed subsets of $B$ is called a support system if it is closed under the formation of closed subsets and finite 
unions. (Note that $B \notin \Phi$ would imply that the complements of sets in $\Phi$ form a filter of open sets.) The set of all sections $\sigma$ in a sheaf $\pi$ whose support $\{b \in B \mid \sigma(b) \neq 0\}$ is in $\Phi$ is denoted by $\Gamma_{\Phi}(\pi, B)$.

(ii) The sheaf $\pi: E \rightarrow B$ is called $\Phi$-soft if every section $X \rightarrow E$ with $X \in \Phi$ extends to a global section.

(iii) If $R$ is a ring and $B \subseteq \operatorname{Spec} R$, and if $M$ is a left $R$-module, then the support system on $B$ of all closed quasi-compact sets $X \cong B$ with (X, e) will be called $\Phi(R)$.

Our previous discussion then yields the following result:

THEOREM 1.12. Let $R$ be a ring, $B$ a space of proper prime ideals and $M a$ left $R$-module. Then there is a $\Phi(R)$-soft sheaf $\mu: E \rightarrow B$ of $R$-modules associated with any family $\left\{K_{I} \mid I \in B\right\}$ of submodules $K_{I}$ of $M$ with $I \cdot M \subseteq K_{I}$ which has the stalks $E_{I} \cong M / \tilde{K}_{I}$ with $\tilde{K}_{I}=\bigcup_{I \in U} \bigcap\left\{K_{J} \mid J \in U\right\}$ (where $U$ ranges over the hull-kernel neighborhoods of I), and the Gelfand morphism $m \mapsto \tilde{m}: M \rightarrow \Gamma(\mu, B)$ given by $\tilde{m}(I)=\left(m+\widetilde{K}_{I}, I\right) \in M / \widetilde{K}_{I} \times\{I\}$ has the kernel $\bigcap\left\{K_{I} \mid I \in B\right\}$; its image contains $\Gamma_{\Phi(R)}(\mu, B)$.

The following corollary perhaps illustrates this result more poignantly:

COROLlarY 1.13. Let $R$ be a ring with identity and $B$ a space of prime ideals containing all maximal ideals. If $M$ is a left $R$-module and $\mu: E \rightarrow B$ the sheaf of $R$-modules in 1.12 , then the sequence

$$
0 \rightarrow \bigcap\left\{K_{I} \mid I \in B\right\} \rightarrow M \rightarrow \Gamma(\mu, B) \rightarrow 0
$$

is exact and $\mu$ is $\Phi$-soft for the collection $\Phi$ of all closed subsets of $B$.

In view of the special nature of the hull-kernel topology we can express the submodules $\widetilde{K}_{I}$ in an alternative version:

1.12.1. $\tilde{K}_{I}=\bigcup_{a \notin I} \bigcap\left\{K_{J} \mid a \notin J\right\}$, where $a$ ranges through elements of $R$.

We apply the theorem and its corollary to the special case that $M=R$. For any subset $A$ of the ring $R$ we call the set $\{I \in B \mid A \nsubseteq I\}$ the support $S(A)$ of $A$ (in $B)$; if we write $S(a)$ in place of $S(\{a\})$, this agrees with previous notation.

We observe that $I \cdot M=I R \subseteq I$. We take $K_{I}=I ;$ and in place of $\tilde{K}_{I}$ we simply write $\tilde{I}$. Thus

$$
\tilde{I}=\bigcup_{a \notin I} \cap S(a), \quad a \in R .
$$

In order to describe $\tilde{I}$ in alternative ways we prove the following lemma, which is due to KeIMEL:

LEMMA 1.14. Let $B$ be an arbitrary collection of prime ideals in a ring $R$, let $I$ be an ideal and $A$ a subset of $R$. Then the following conditions are 
equivalent :

(1) $I=\bigcap S(A)$.

(2) $I$ is the largest ideal with $A I \subseteq \bigcap B$.

(3) $I$ is the largest ideal with $I A \subseteq \bigcap B$.

(4) $I$ is the largest ideal with $A I+I A \subseteq \bigcap B$.

(5) $I$ is the largest ideal with $(A) \cap I \subseteq \bigcap B$.

REMARK. Recall that $\bigcap S(A)=\bigcap\{\tilde{I} \mid A \nsubseteq I\}$ by 1.5 .

Proof. Assume (2), take $P \in S(A)$. Now $A I \subseteq \bigcap B$ implies $A I \subseteq(A) I$ $\subseteq \bigcap B \subseteq P$ on one hand; from $P \in S(A)$ we have $(A) \nsubseteq P$ on the other. Since $P$ is prime, then $I \subseteq P$. Hence $I \subseteq \bigcap S(A)$. Converse inclusion: If $P \in B$ and $A \nsubseteq P$, then $P \in S(A)$, and so $A(\bigcap S(A)) \subseteq \bigcap S(A) \subseteq P$; if, however, $A \subseteq P$ then $A(\bigcap S(A)) \subseteq P(\bigcap S(A)) \subseteq P$. Consequently $A(\bigcap S(A))$ $\subseteq \bigcap B$, and thus $\bigcap S(A) \subseteq I$. This shows (1).

Assume (1). If $P \in B$, then $P \in S(A)$ implies $A I \subseteq I=\bigcap S(A) \subseteq P$, and $P \notin S(A)$ implies $A \subseteq P$, hence $A I \subseteq P I \subseteq P$; thus $A I \subseteq \bigcap B$. Suppose that $A J \subseteq \bigcap B$; then $(A) J \subseteq P$ for all $P \in B$, in particular for all $P \in S(A)$; but for these we have $(A) \nsubseteq P$ and so $J \subseteq P$ since $P$ is prime. Hence $J \subseteq \bigcap S(A)=I$. This shows (2).

Since (1) is completely symmetric, we also have the equivalence of (1) and (3). Thus (1) also implies (4), but (4) implies (2). Since $A I \subseteq(A) \cap I$, then $(A) \cap I \subseteq \bigcap B \Rightarrow A I \subseteq \bigcap B \Rightarrow((A) \cap I)^{2} \subseteq(A) I \subseteq \bigcap B$; but $R / \cap B$ is semiprime and thus does not contain any nilpotent ideals, whence $(A) \cap I \subseteq \bigcap$, thus $(A) \cap I \subseteq \bigcap B \Leftrightarrow A I \subseteq \bigcap B$, and the lemma is proved.

We will call the ideal $I$ of 1.14 the annihilator of $A$ modulo $\cap B$ and write $I=A_{B}^{\perp}$. If $A=\{a\}$ we abbreviate $\{a\}_{B}^{\perp}$ to $a_{B}^{\perp}$. The ideal $\bigcap B$ will be called the $B$-radical, written $\bigcap B=B$ - $\operatorname{Rad} R$. If $B$ is the space of all maximal ideals we write $B=\operatorname{Max} R$.

THEOREM 1.15. Let $R$ be a ring, $B$ a space of prime ideals, and $\Phi$ the support system of all subsets $X \subseteq B$ satisfying

(a) $X$ is closed;

(b) there is a relative identity $e \in R$ for $\bigcap X$;

(c) any ideal $I$ of $R$ with $\bigcap X \cong I$ is contained in some $J \in X$.

Then there is a sheaf $\rho: E \rightarrow B$ of rings with stalks $E_{I}=R / \tilde{I} \times\{I\}$ $\cong R / \tilde{I}$ with $\tilde{I}=\bigcup_{a \notin I} a_{B}^{\perp}, a \in R$ such that the following conditions are satisfied:

(i) The ring morphism $a \mapsto \tilde{a}: R \rightarrow \Gamma(\rho, B)$ with $\tilde{a}(I)=(a+\tilde{I}, I)$ has $B-\operatorname{Rad} R$ as its kernel, and its image contains $\Gamma_{\Phi}(\rho, B)$.

(ii) The sheaf $\rho$ is $\Phi$-soft.

Note that all $X \in \Phi$ are quasi-compact, and that (c) is automatic if $\operatorname{Max} R$ $\subseteq B$. 
Again the resulting representation theorem for rings with identity shows more clearly what goes on:

COROLlary 1.16. Let $R$ be a ring with identity and $B$ a space of prime ideals containing all maximal ideals. Then there is a sheaf of rings with identity $\rho: E \rightarrow B$ with stalks $E_{I}=R / \tilde{I}$ with $\tilde{I}=\bigcup_{a \neq I} a_{B}^{\perp}, a \in R$ such that

(i) the sequence $0 \rightarrow B-\operatorname{Rad} R \rightarrow R \rightarrow \Gamma(\rho, B) \rightarrow 0$ is exact,

(ii) the sheaf $\rho$ is $\Phi$-soft for the set $\Phi$ of all closed subsets of $B$.

Recall that a ring $R$ with identity is called semiprime if the prime radical (Spec $R$ )- $\operatorname{Rad} R=\bigcap \operatorname{Spec} R$ is (0) with the space Spec $R$ of all prime ideals. We immediately obtain the following representation theorem for semiprime rings:

THEOREM 1.17. Any semiprime ring $R$ with identity is isomorphic to the ring of all global sections in a sheaf $\rho: E \rightarrow$ Spec $R$ of rings whose stalks are given by $E_{I} \cong R / \tilde{I}, \tilde{I}=\bigcup_{a \notin I} a^{\perp}$ where $a^{\perp}$ is the largest ideal annihilated by a on the left and right.

A ring is called semisimple, resp., strongly semisimple, if the Jacobson radical $(\operatorname{Prim} R)-\operatorname{Rad} R=\bigcap \operatorname{Prim} R$ with the space Prim $R$ of all primitive ideals (resp. the strong radical $(\operatorname{Max} R)-\operatorname{Rad} R=\bigcap \operatorname{Max} R$ ) is (0). We immediately derive the following version of the representation theorem:

COROLlaRY 1.18. Any semisimple (resp., strongly semisimple) ring $R$ with identity is isomorphic to the ring of all global sections in a sheaf $\rho: E$ $\rightarrow \operatorname{Prim} R$ (resp., $\rho: E \rightarrow \operatorname{Max} R$ ) whose stalks are given by $E_{I} \cong R / \tilde{I}, \tilde{I}$ $=\bigcup_{a \notin I} a^{\perp}$, where $a^{\perp}$ is the largest ideal annihilated by $a$ on the left and right.

Let us introduce the following definition:

Definition 1.19. For any ring $R$ and any space $B$ of prime ideals denote by $\mathscr{R}$ the sheaf $\Gamma(\rho, ?): \mathcal{O}(B)^{\text {op }} \rightarrow B$ which we canonically constructed in 1.15. For any left $R$-module $M$ we denote by $\mathscr{M}$ the sheaf $\Gamma(\mu, ?): \mathcal{O}(B)^{\text {op }}$ $\rightarrow B$, where $\mu$ is the sheaf constructed as in 1.12 with $K_{I}=I \cdot M$.

It is now easily observed that there is a bilinear sheaf map $\mathscr{R} \times \mathscr{M} \rightarrow \mathscr{M}$ which is defined as follows: Let $U \in \mathcal{O}(B)$ and $(\sigma, \tau) \in \mathscr{R}(U) \times \mathscr{M}(U)$ then $\sigma \cdot \tau \in \mathscr{M}(U)$ is defined by $(\sigma \cdot \tau)(I)=\sigma(I) \cdot \tau(I)$ where the scalar multiplication $\rho^{-1}(I) \times \mu^{-1}(I) \rightarrow \mu^{-1}(I)$ in the stalks over $I$ is well defined by the prescription $(a+\tilde{I}) \cdot\left(m+(I \cdot M)^{\mathcal{2}}=a \cdot m+(I \cdot M)^{\mathcal{2}}\right.$ (indeed one has to check that, firstly $A \cdot(I \cdot M)^{\sim} \subseteq(I \cdot M)^{\sim}$, which is trivial because of the fact that $(I \cdot M)^{\sim}$ is a submodule, and, secondly, that $\tilde{I} \cdot M \subseteq(I \cdot M)^{\sim}$ which is perhaps not trivial, but nevertheless straightforward from the definitions). This bilinear map makes $\mathscr{M}(U)$ into a left $\mathscr{R}(U)$-module. The geometric version of the scalar operation of $\mathscr{R}$ on $\mathscr{M}$ is given as 
follows: Let $\rho: E \rightarrow B$ and $\mu: G \rightarrow B$ be the sheaf projections of $\mathscr{R}$ and $\mathscr{M}$, respectively. Let $\rho \times{ }_{B} \mu: E \times{ }_{B} G \rightarrow B\left(\right.$ with $E \times{ }_{B} G=\{(x, y) \mid \rho(x)=\mu(y)\}$ ) the product sheaf projection; then there is a continuous map $E \times{ }_{B} G \rightarrow G$ which is bilinear on stalks and makes each stalk $G_{I}=M /(I \cdot M)^{\sim}$ into a left $R / \tilde{I}$-module.

DEFINITION 1.20 . Let $\mathscr{R}$ be a sheaf of rings with identity and $\mathscr{M}$ a sheaf of abelian groups. We say that $\mathscr{M}$ is a left $\mathscr{R}$-module if there is a bilinear map $\mathscr{R} \times \mathscr{M} \rightarrow \mathscr{M}$ which makes each $\mathscr{M}(U)$ into a left $\mathscr{R}(U)$-module.

With this convenient definition we have

Proposition 1.21. For any ring $R$ with identity and any space $B$ of prime ideals containing the maximal ideals and for any left $R$-module $M$ we obtain $a$ left $\mathscr{R}$-module $\mathscr{M}$ whose properties are given in Corollaries 1.13 and 1.16.

$A$ second construction for rings. The ideas of 1.14 motivate a slightly modified construction of a sheaf associated with an $R$-module, $M$. For a subset $A \subseteq R$, let $A^{\perp}=\{m \in M \mid(A) \cdot m=(0)\}$ be the largest submodule of $M$ annihilated by $A$. For $\{a\}^{\perp}$ we write $a^{\perp}$. Since $a^{\perp}+b^{\perp} \subseteq(a b)^{\perp}$, the family $\left\{a^{\perp} \mid a \notin I\right\}$ is directed relative to $\subseteq$ for every prime ideal $I$; therefore $\check{I}=\bigcup_{a \notin I} a^{\perp}$ is a submodule of $M$. If $m \in \check{I}$, there is an $a \notin I$ with $m \in a^{\perp}$, and $a^{\perp} \subseteq J$ for all $J$ with $a \notin J$, i.e. $J \in S(a)$. Then we can construct a sheaf $\mu^{\prime}: E^{\prime} \rightarrow B$ also written $\mathscr{M}^{\prime}$, where $E_{I}^{\prime} \cong M / \check{I}$ (say $E_{I}^{\prime}=M / \check{I} \times\{I\}$ ) and $\mu^{\prime}(m+\breve{I}, I)=I$; the topology on $E^{\prime}$ is the finest making all functions $\hat{m}: B$ $\rightarrow E^{\prime}$ with $\hat{m}(I)=(m+\check{I}, I)$ continuous. The $R$-module morphism $\hat{m} \mapsto m: M \rightarrow \Gamma\left(\mu^{\prime}, B\right)$ has the kernel $\bigcup\{\breve{I} \mid I \in B\}$. Suppose now that $m \in \check{I}$ for all $I \in B$. If every annihilator ideal of a cyclic module in $M$ is contained in some ideal of $B$, then the annihilator ideal of $R \cdot m$ is contained in some $I \in B$. But $m \in \bigcap\{J \mid \breve{J} \in B\} \subseteq \breve{I}$, so there is an $a \notin I$ with $m \in a^{\perp}$, whence $(a) \cdot m=(0)$. Then $(a)$ is in the annihilator of $R \cdot m$ and thus $a \in(a) \subseteq I$, a contradiction. Thus we have the following lemma, due to $\mathrm{KoH}$ :

LEMMA 1.22. Let $M$ be a left $R$-module such that every annihilator ideal of a cyclic submodule $R \cdot m \cong M$ is contained in some ideal of $B$. Then $m \mapsto \hat{m}: M \rightarrow \Gamma\left(\mu^{\prime}, B\right)$ is injective.

In general, the image of $M \rightarrow \Gamma\left(\mu^{\prime}, B\right)$ will not contain $\Gamma_{\Phi(R)}\left(\mu^{\prime}, B\right)$. However, if we introduce the subsheaf $\mathscr{M}^{\prime \prime}$ of $\mathscr{M}^{\prime}$ whose sheaf space is the interior of $\bigcup\{I \cdot(M / \check{I} \times\{I\}) \mid I \in B\}$, then the method used in the proof of 1.12 will show that the image contains $\Gamma_{\Phi(R)}\left(\mu^{\prime}, B\right)$ modulo $\mathscr{M}^{\prime \prime}(B)$.

In particular, we have the following result:

Proposition 1.23. Let $R$ be a ring with identity and $B$ a space of prime ideals with $\operatorname{Max} R \subseteq B$. Let $M$ be a left $R$-module. Then there is a sheaf $\mathscr{M}^{\prime}$ of $R$-modules over $B$ with stalks isomorphic to $M / \check{I}$, where $\check{I}=\bigcup_{a \notin I} a^{\perp}, a \in R$, and where $a^{\perp}$ is the largest submodule of $M$ annihilated by $a$. The Gelfand 
morphism $m \mapsto \hat{m}: M \rightarrow \mathscr{M}^{\prime}(B)$ is an injection. Let $\mathscr{M}^{\prime \prime}$ be the subsheaf of $\mathscr{M}^{\prime}$ whose sheaf space is the interior of $\bigcup\left\{I \cdot E_{I}^{\prime} \mid I \in B\right\}$, where $E^{\prime}$ is the sheaf space of $\mathscr{M}^{\prime}$. Then $\mathscr{M}^{\prime}(B)=\hat{M}+\mathscr{M}^{\prime \prime}(B)$ and $\hat{M} \cong M$.

The most important case, however, is the case that $M=R$. Then $a^{\perp}$ is the largest ideal $J$ of $R$ such that $(a) J=(0)$. Thus $a^{\perp} \subseteq a_{B}^{\perp}$ (see $1.14 \mathrm{ff}$.); if $B-\operatorname{Rad} R=(0)$, then $a^{\perp}=a_{B}^{\perp}$. It follows that $\breve{I}=\bigcup_{a \notin I} a^{\perp}$ is contained in $\tilde{I}=\bigcup_{a \notin I} a_{B}^{\perp}$. Let us now denote by $\mathscr{R}^{\prime}$ the sheaf $\rho^{\prime}: E^{\prime} \rightarrow B$ with stalks $E_{I}^{\prime}=R / I \times\{I\}$ constructed above. The Gelfand morphism $R \rightarrow \Gamma\left(\rho^{\prime}, B\right)$ $=\mathscr{R}^{\prime}(B)$ is then injective by 1.22 . The quotient morphisms $\varphi_{I}: R / \check{I} \rightarrow R / \tilde{I}$ induce a sheaf morphism $\varphi: \mathscr{R}^{\prime} \rightarrow \mathscr{R}$ which may be considered as a local homeomorphism $\varphi: E^{\prime} \rightarrow E$ of the respective sheaf spaces with $\rho^{\prime}=\rho \varphi$. Then $\varphi$ maps $\bigcup\left\{I \cdot E_{I}^{\prime} \mid I \in B\right\}$ onto the $\operatorname{radical} \operatorname{Rad} E=\bigcup\{I / \tilde{I} \times\{I\} \mid I \in B\}$ of $\rho$; since $\varphi$ is a local homeomorphism, the interior of the former space is then exactly $\varphi^{-1} \mathcal{O}(B)=\bigcup\{\tilde{I} / I \times\{I\} \mid I \in B\}$ in view of 1.9. This interior is the sheaf space of a subsheaf $\mathscr{R}^{\prime \prime}$ of $\mathscr{R}^{\prime}$, and $0 \rightarrow \mathscr{R}^{\prime \prime} \rightarrow \mathscr{R}^{\prime} \rightarrow \mathscr{R} \rightarrow 0$ is an exact sequence of sheaves of rings.

While in general we cannot say very much about the image of $R$ in $\mathscr{R}^{\prime}(B)$ there are certain conditions under which additional conclusions may be drawn. We discuss briefly one such set of conditions. In KeIMEL's Lemma 1.14 together with 1.5 we had the conclusion

$$
A_{B}^{\perp}=\bigcap\{\tilde{I} \mid A \nsubseteq I\} \quad \text { for all } A \cong R .
$$

We now postulate the following condition:

$$
A^{\perp}=\bigcap\{\check{I} \mid A \notin I\} \quad \text { for all } A \cong R .
$$

Notice that $a \notin I$ implies $a^{\perp} \subseteq \check{I}$ by the definition of $\breve{I}$; now $A \nsubseteq I$ implies the existence of an $a \in A \backslash I$, hence $A^{\perp} \subseteq a^{\perp} \subseteq \check{I}$; thus the inclusion $\subseteq$ is always satisfied in 1.24.2. Furthermore, $A^{\perp}=\bigcap\left\{a^{\perp} \mid a \in A\right\}$; hence 1.24.2 is in fact equivalent to

$$
\bigcap\{\check{I} \mid a \notin I\} \subseteq a^{\perp} \quad \text { for all } a \in R .
$$

Suppose that $R$ has an identity, whence $B$ is quasi-compact. Now let $\sigma: B \rightarrow E^{\prime}$ be a section. By the usual compactness argument which we used in the discussion of 1.12 we find elements $a_{k}, m_{k}, k=1, \ldots, n$, in $R$ such that with $U_{k}=S\left(a_{k}\right)$ we have $\sigma\left|U_{k}=\hat{m}_{k}\right| U_{k}$, and that $B \subseteq U_{1} \cup \cdots \cup U_{n}$. Because of the latter relation, if we assume that every proper ideal of $R$ is contained in some ideal of $B$, it follows that $R=\left(a_{1}\right)+\cdots+\left(a_{n}\right)$. Thus the identity 1 may be written in the form $1=r_{1}+\cdots+r_{n}$ with $r_{k} \in\left(a_{k}\right)$ for $k=1, \ldots, n$.

We define $a=r_{1} m_{1}+\cdots+r_{n} m_{n}$ and claim $\hat{a}=\sigma$. Indeed, firstly we observe that $\left(\hat{m}_{i}-\hat{m}_{k}\right)(I)=\sigma(I)-\sigma(I)=0$ for $I \in S\left(a_{k}\right) \cap S\left(a_{i}\right)$ 
$=S\left(\left(a_{k}\right)\left(a_{i}\right)\right)$; hence $m_{i}-m_{k} \in \bigcap\left\{I \mid I \in S\left(\left(a_{k}\right)\left(a_{i}\right)\right)\right\}=\left[\left(a_{k}\right)\left(a_{i}\right)\right]^{\perp}$ by hypothesis 1.24.2. Thus $\left(a_{k}\right)\left(a_{i}\right)\left(m_{i}-m_{k}\right)=(0)$ and therefore $\left(a_{k}\right)\left(a-m_{k}\right)$ $=\left(a_{k}\right)\left(\sum_{1}^{n} r_{i}\left(m_{i}-m_{k}\right)\right)=(0)$.

Hence $a-m_{k} \in a_{k}^{\perp} \in I$ for every $I \in S\left(a_{k}\right)$ by the definition of $\check{I}$. If we now, finally, take an arbitrary $I \in B$, then $I \in S\left(a_{k}\right)$ for some $k$ and we have $(\hat{a}-\sigma)(I)=\hat{a}(I)-\sigma(I)=\hat{a}(I)-\hat{m}_{k}(I)=0$.

It is not entirely clear how this process should be generalized in the case of the absence of an identity in such a fashion that relative identities might serve in a role analogous to the one that had in 1.15.

Let us remark that now in view of the faithful representation of $R$ in $\mathscr{R}^{\prime}(B)$, condition 1.24 .3 may be reinterpreted as follows:

We write $\operatorname{Rad} E^{\prime}=\bigcup\{I / \check{I} \times\{I\} \mid I \in B\} \cong E$ and call $\{I \in B \mid \sigma(I)$ $\left.\notin \operatorname{Rad} E^{\prime}\right\}$ the quasi-support of a global section $\sigma \in \mathscr{R}^{\prime}(B)$. Thus 1.24 .3 is equivalent to

1.24.4. For $a, b, c$ in $R$, if $\hat{c}$ vanishes on the quasi-support of $\hat{a}$, then $\hat{a} \hat{b} \hat{c}=0$ (i.e. $a b c=0$ ).

Note that unlike the support, the quasi-support is not closed. The fact that $\hat{c}$ vanishes on the quasi-support of $\hat{a}$ may be expressed as quasi-supp $\hat{a}$ $\cap \operatorname{supp} \hat{c}=\varnothing$.

The key theorem. We now have the following key theorem.

THEOREM 1.24. (a) Let $R$ be a ring and $B$ a space of prime ideals. Then there is an exact sequence $0 \rightarrow \mathscr{R}^{\prime \prime} \rightarrow \mathscr{R}^{\prime} \rightarrow \mathscr{R} \rightarrow 0$ of sheaves of rings where $\mathscr{R}_{I}^{\prime \prime}=\tilde{I} / \check{I}, \mathscr{R}_{I}^{\prime}=R / \check{I}, \mathscr{R}_{I}=R / \tilde{I}$ with $\check{I}=\bigcup_{a \neq I} a^{\perp}, \tilde{I}=\bigcup_{a \notin I} a_{B}^{\perp}$, where $a^{\perp}$ (resp. $a_{B}^{\perp}$ ) is the largest ideal $J$ of $R$ with $(a) J \subseteq(0)$ (resp. $\cap B$ ). If for every $a \in R$ there is an $I \in B$ with $a^{\perp} \subseteq I$, then we have a commutative diagram

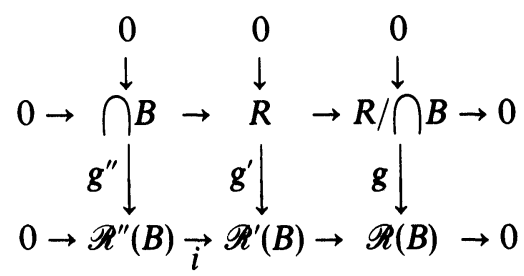

with exact rows and columns. The image of $g$ contains $\Gamma_{\Phi(R)}(\rho, B)($ see 1.11), and $\Gamma_{\Phi(R)}\left(\rho^{\prime}, B\right) \subseteq \operatorname{im} g^{\prime}+\operatorname{im} i$.

(b) If $R$ has an identity and $B$ contains Max $R$ then $g$ is an isomorphism and the left square in the diagram is a pullback and pushout (i.e. $\operatorname{ker} i \cap$ $\operatorname{ker} \boldsymbol{g}^{\prime} \cong \bigcap \boldsymbol{B}$ and $\operatorname{im} i+\operatorname{im} g^{\prime}=\mathscr{R}^{\prime}(B)$ ).

(c) If under the hypotheses of (b) we have the additional condition that $a^{\perp} \supseteqq \bigcap\{\check{I} \mid a \notin I\}$ for all $A \subseteq R$, then $g^{\prime}$ is an isomorphism, too; equivalently $\mathrm{g}^{\prime \prime}$ is an isomorphism. 
The following example illustrates that not much more improvement over 1.24 even in (b) and (c) may be expected. Let $K$ be a field and $R$ the ring of all matrices $a$ of the form

$$
\left[\begin{array}{ll}
u & v \\
0 & w
\end{array}\right]
$$

Then $\operatorname{Spec} R$ consists of two isolated points $I$ and $J$, where $I$ contains all elements with $w=0$ and $J$ those with $u=0$. The radical is $I \cap J$, i.e. the set of all $a$ with $u=w=0$. Since $I$ and $J$ are isolated, then with $B=\operatorname{Spec} R$ we obtain $\tilde{I}=I$ and $\tilde{J}=J$, and thus $R / \tilde{I}=R / \tilde{J}=K, \mathscr{R}(B)=K \times K$. Straightforward computation shows that $I^{\perp}=\breve{J}=(0)$, but that $J^{\perp}=\check{I}$ $=I$ (which illustrates that $A^{\perp}$ is defined asymmetrically for $A \subseteq R$ ). Thus $\mathscr{R}^{\prime}(B)=K \times R$ and $\mathscr{R}^{\prime \prime}(B)=J$. Hence $\mathscr{R}^{\prime \prime}(B)$ is not the radical of $\mathscr{R}^{\prime}(B)$ and $\hat{R} \neq \mathscr{R}^{\prime}(B)$. Note that indeed condition 1.23 .3 is violated with $a=\left[\begin{array}{ll}0 & 1 \\ 0 & 0\end{array}\right]$, since then $a^{\perp}=I$, whereas there is no $P \in \operatorname{Spec} R$ with $a \notin P$, whence $\bigcap\{P \mid a \in I\}=R \nsubseteq I=a^{\perp}$.

In $\$ 2$ we will see that 1.24 .3 is satisfied for commutative rings with $B=\operatorname{Spec} R$.

Sheaves of local rings. We now discuss the question under which circumstances the stalks of the sheaf $\mathscr{R}$ are local rings. For a space $B$ of prime ideals of a ring $R$ the hull $h(A)=h_{B}(A)$ (relative to $B$ ) of a subset $A \subseteq R$ is the set $\{I \in B \mid A \subseteq I\}$. Recall $\tilde{I}=\bigcup_{a \notin I} \bigcap S(a)=\bigcup_{a q I} a_{B}^{\perp}$ and $\check{I}=\bigcup_{a \notin I} a^{\perp}$.

LEMMA 1.25. Let $B \subseteq \operatorname{Spec} R$. Then

$$
\begin{aligned}
h_{B}(\tilde{I}) & =\bigcap\{\bar{U} \mid U \text { a neighborhood of } I \text { in } B\}=\bigcap\left\{S(a)^{-} \mid a \notin I\right\} \\
& =\bigcap\left\{h_{B}\left(a_{B}^{\perp}\right) \mid a \notin I\right\}
\end{aligned}
$$

and $h_{B}(\check{I})=\bigcap\left\{h\left(a^{\perp}\right) \mid a \in I\right\}$ (all hulls and $S(a)$ taken relative to $B$ ).

Proof. We have $J \in h\left(a_{B}^{\perp}\right)$ iff $a_{B}^{\perp} \subseteq J$ iff $\bigcap S(a) \subseteq J$ (by 1.14) iff $J \in S(a)^{-}$ for all $J \in B$. This is the case for all $a \notin I$ iff $J \in h(\tilde{I})$. Since the $S(a), a \notin I$ form a neighborhood basis for $I$ in $B$, the first chain of equalities is proved. The second equality is similar.

We will call a ring local if it has exactly one maximal proper ideal containing all proper ideals (note carefully that some authors postulate an identity and that the unique maximal ideal is the set of nonunits). Thus, if $R$ is a ring then $R / \tilde{I}$ is local iff $\bigcap\left\{S_{\mathrm{Spec} R}(a)^{-} \cap \operatorname{Max} R \mid a \notin I\right\}$ is singleton. Further $R / \check{I}$ is local iff $\bigcap\left\{h_{\text {Spec } R}\left(a^{\perp}\right) \cap \operatorname{Max} R \mid a \notin I\right\}$ is singleton.

Definition 1.26. We say that the inclusion $X \rightarrow Y$ of a subspace of a topological space is a Hausdorff embedding if two different points of $X$ have disjoint neighborhoods in $Y$.

We are now in a position to prove the following

Proposition 1.27. Let $R$ be a ring and B a space of proper prime ideals. 
Assume that $B$ contains all $M \in \operatorname{Max} R$ for which there is an $I \in B$ with $I \subseteq M$; then the following conditions are equivalent:

(a) $\bigcap\left\{S(a)^{-} \mid a \notin I\right\} \cap \operatorname{Max} R$ is singleton for all $I \in B$, where $S(a)$ $=\{J \in \operatorname{Spec} R \mid a \notin J\}$.

(b) $\mathscr{R}$ is a sheaf of local rings.

(c) For each $I \in B$ there is a unique $\mu(I) \in B \cap \operatorname{Max} R$ with $I \subseteq \mu(I)$ and $B \cap \operatorname{Max} R \rightarrow \operatorname{Spec} R$ is a Hausdorff embedding.

Proof. By the above (a) $\Leftrightarrow$ (b). Suppose (a). Clearly, $\mu$ is well defined. If $M, N \in B \cap \operatorname{Max} R$ and if $M \neq N$, then there is an $a \notin M$ such that $N \notin S(a)^{-}$; thus (c). Suppose (c). Let $I \in B$ and $N \in B \cap \operatorname{Max} R$ with $N \neq \mu(I)$. Then there is a closed neighborhood $U$ of $\mu(I)$ in Spec $R$ not containing $N$; there is an $a \notin \mu(I)$ such that $S(a)^{-} \subseteq U$, and $I \in S(a)$ because of $I \subset \mu(I)$. Hence $N$ is not contained in the intersection of all closed neighborhoods of $I$ in $\operatorname{Spec} R$. This proves (a).

In particular this yields the following result of $\mathrm{KoH}:$ If $B$ is contained in Max $R$ then $\mathscr{R}$ is a sheaf of local rings iff $B$ is Hausdorff.

If the conditions of (a), (b), or (c) of 1.27 are satisfied, then there is a welldefined retraction $\mu: B \rightarrow B \cap \operatorname{Max} R$, where $\mu(I)$ is the unique maximal ideal containing $I$. This function may not be continuous; one can show, as KEIMEL and the author did in [45], that it is continuous if every point $I \in B \cap \operatorname{Max} R$ has a basis of closed neighborhoods in $B$; such maximal ideals are called regular. What we do know on the basis of (a) is that for any $I \in B \cap \operatorname{Max} R$, the closed neighborhoods of $I$ intersect in $\{I\}$. If $B$ is quasicompact, then this suffices for $I$ to be regular. Under these circumstances every continuous function of $B$ into a Hausdorff space factors through $\mu$; since $B \cap \operatorname{Max} R$ is a maximal Hausdorf subspace of $B$, then $\mu$ is the universal Hausdorffization and, because of the compactness of $B \cap \operatorname{Max} R$ under our circumstances, in fact the Stone-Čech compactification. We may thus add to 1.27 the following additional

REMARK. If the hypotheses and the equivalent conditions (a), (b), (c) of 1.27 are satisfied and if, in addition, $B$ is quasi-compact, then $\mu: B \rightarrow B$ $\cap \operatorname{Max} R$ is a continuous retraction and is, in fact, the Stone-Cech compactification of $B$.

The example following 1.24 shows that $\mathscr{R}^{\prime}$ need not be a sheaf of local rings even if $R$ has an identity and $B=\operatorname{Max} R$ is Hausdorff. In $\$ 2$ we will see that for a commutative ring $R$ with identity, $\mathscr{R}^{\prime}$ is a sheaf of local rings iff .7 is one.

It should nevertheless be noted that, from an algebraic point of view, the ideals $\check{I}=\bigcup_{a \notin I} a^{\perp}$ with the annihilator $a^{\perp}$ of $a$ in $R$ are generally more likely to yield local rings $R / \check{I}$ than the ideals $\tilde{I}$ (but recall that $a_{B}^{\perp}=a^{\perp}$ if $\bigcap B=(0) !)$ : Indeed suppose that $J$ is an ideal of $R$ such that $R / J$ is a local ring, with maximal proper ideal $I / J$, and suppose that every proper ideal 
of $R$ is contained in a maximal proper ideal. If $a \notin I$, then by this hypothesis, the ideal $(a)+J$ cannot be proper, since $I$ is the only proper maximal ideal containing $J$. Hence $R=(a)+J$. If the local ring $R / J$ satisfies $(R / J)^{\perp}=0$ (which is the case if it has an identity, notably if $R$ has an identity), then $a^{\perp} \subseteq J$. Hence we have the following observation, due to $\mathrm{KoH}$ [57]:

If every proper ideal of $R$ is contained in a maximal one, and if $J \subseteq I$ are ideals such that $R / J$ is local with maximal proper ideal $I / J$ and with $(R / J)^{\perp}=0$, then $\breve{I} \subseteq J$. In particular, if $R / \breve{I}$ is local, then $\breve{I}$ is the unique minimal ideal $J$ of $R$ in $I$ such that $R / J$ is local. (Such ideals are called I-primary.)

Teleman calls a ring $R$ harmonic, if $B=\operatorname{Max} \operatorname{Mod} R$ (the space of maximal modular ideals ) is locally compact Hausdorff and if for each $I \in B$ there is a neighborhood $U$ of $I$ in $B$ such that there is an identity $e$ of $r$ modulo any $J \in U$.

If $R$ has an identity, it is harmonic iff Max $R$ is compact Hausdorff, and in any case $B \subseteq \operatorname{Max} \operatorname{Mod} R$ is locally compact Hausdorff if $R$ is harmonic. Teleman has given a thorough theory for the sectional representation of harmonic rings, for which his Tulane Lecture Notes are an excellent source of reference [92]. KоH [58] calls a ring $R$ strongly harmonic, if for any pair $I, J$ of different maximal modular ideals of $R$ there exist ideals $I^{\prime}, J^{\prime}$ in $R$ with $I^{\prime} J^{\prime}=(0)$ and $I^{\prime} \nsubseteq I, J^{\prime} \nsubseteq J$. This is clearly tantamount to saying that there is an element $a \notin I$ with $a^{\perp} \nsubseteq J$. Thus a ring $R$ is strongly harmonic iff for different maximal modular ideals $I$ and $J$ we have $\breve{I} \nsubseteq J$. Then $B=\operatorname{Max} \operatorname{Mod} R$ is Hausdorff, and the sheaf $\mathscr{R}^{\prime}$ is a sheaf of local rings in the sense that $\mathscr{R}_{x}^{\prime}=R / \check{I}$ has the unique maximal modular ideal $I / \check{I}$; by preceding comments, $\check{I}$ is then $I$-primary. The particular feature which distinguishes strongly harmonic rings in the context of sectional representation, however, has been observed by $\mathrm{KoH}[58$ ]: If $B=\operatorname{Max} \operatorname{Mod} R$ and $X \subseteq B$ is compact, then for any $I \notin X$ there is an ideal $A$ of $R$ with $X \subseteq S(A)$ and $A^{\perp} \nsubseteq I$ : Indeed if $I \notin X$ and $J \in X$ then there is an $a_{J} \notin I$ with $J \in S\left(a_{J}^{\perp}\right)$ by definition of strong harmonicity. By a compactness argument we find $a_{1}, \ldots, a_{n} \notin I$ such that $X \subseteq S\left(a_{1}^{\perp}\right) \cup \cdots \cup S\left(a_{n}^{\perp}\right)$; take $A=a_{1}^{\perp}+\cdots+a_{n}^{\perp}$, then $X \subseteq S(A)$ and $\left(a_{1}\right) \cdots\left(a_{n}\right) \subseteq A^{\perp} \nsubseteq I$. Now suppose $M \in B$ and $\bigcap\{\check{I} \mid I \in X\} \subseteq M$. If $M$ were not in $X$, then by the preceding we could find an ideal $A$ with $X \subseteq S(A)$ and $A^{\perp} \nsubseteq M$; if $I \in X$ is arbitrary, then $A \nsubseteq I$, hence there is an $a \in A \backslash I$, whence $A^{\perp} \subseteq a^{\perp} \subseteq I$. Thus $A^{\perp}$ $\subseteq \bigcap\{\check{I} \mid I \in X\} \subseteq M$, a contradiction. Therefore, for any compact $X \subseteq B$ we have

$$
X=h(\bigcap\{\check{I} \mid I \in X\}) .
$$

This enables us to produce exceptionally good partitions of identity: If $X \subseteq U_{1} \cup \cdots \cup U_{n}$ is a finite cover of $X$ by open sets of $B$, define 
$I_{k}=\bigcap\left\{\check{I} \mid I \in X \backslash U_{k}\right\}$; suppose that $e$ is an identity modulo $\bigcap X$ and assume condition $(\mathrm{X})$ of 1.9. Then, as in 1.10 we obtain elements $r_{k} \in I_{k}$ such that $e \equiv r_{1}+\cdots+r_{n} \bmod \bigcap X$.

With such a partition of identity it is an easy exercise to show that every continuous section over $X$ in the sheaf $\mathscr{R}^{\prime}$ is the restriction of a section of the form $\hat{r}, r \in R$. Moreover, if $R$ has an identity, the same argument shows that the Gelfand morphism is surjective. One thus obtains the following theorem:

THEOREM $1.28(\mathrm{KOH}) . A$ ring with identity is strongly harmonic if and only if the sheaf ' $\mathscr{R}^{\prime}$ over the space Max $R$ of all maximal ideals is a sheaf of local rings with identity over a compact Hausdorff space. Under these circumstances $R$ is isomorphic to $\mathscr{R}^{\prime}(\operatorname{Max} R)$ under the Gelfand morphism and the sheaf $\mathscr{R}^{\prime}$ is $\Phi$-soft for the support-system $\Phi$ of all closed subspaces of $\operatorname{Max} R$.

Once it is established with Teleman, that in a sheaf of local rings with identity over a compact Hausdorff space the base space is naturally isomorphic to the maximal ideal space of the ring of global sections, it is not difficult to observe that, conversely, the ring of global sections in any $\Phi$-soft sheaf of local rings with identity over a compact Hausdorff space is strongly harmonic (TELEMAN).

If we very briefly turn back to the key Theorem 1.24 (and the following example) we can rephrase in essence КоH's Theorem 1.28 by saying that for strongly harmonic rings just as in the case of $1.24(\mathrm{c}), g^{\prime}$ as well as $g$ is an isomorphism; equivalently, $g^{\prime \prime}$ is an isomorphism; this we can express by saying that, for a strongly harmonic ring $R$ with identity and the space $B$ of maximal ideals of $R$, the "subring $\mathscr{R}^{\prime \prime}(B)$ " of $\mathscr{R}(B)$ is exactly its strong radical. Outside 1.24 (c) and outside the commutative case (to be discussed in §2) this is the only instance known to me where, perhaps somewhat mysteriously, the radical shows up identifiably in the standard sheaf $\mathscr{R}^{\prime}$. We know that in the sheaf $\mathscr{R}$ it never can.

Teleman has applied sectional representation techniques to various classes of harmonic rings. As an example only let us mention the class of von Neumann regular rings which are continuous (i.e. whose lattice of right principal ideals is continuous ) [87]. In these rings $\operatorname{Max} R$ is Hausdorff embedded into Spec $R$, and if $Z$ denotes the center of $R$, then $M \mapsto M \cap Z$ : $\operatorname{Max} R \rightarrow \operatorname{Max} Z$ is a homeomorphism [87]. Since such rings are strongly semisimple, $\mathscr{R}^{\prime}=\mathscr{R}$ and $R$ is isomorphic to $\mathscr{R}(\operatorname{Max} R)$, and $\mathscr{R}$ is a sheaf of local rings over a Boolean space, since $\operatorname{Max} Z$ is a Boolean space as we shall see in 1.30 and 1.31 below.

As a next step for further specialization we discuss the relation $I=\tilde{I}$ for a prime ideal $I \in B$. Recall that a topological space is zero dimensional if its 
topology has a basis of open and closed sets. Clearly every zero dimensional $T_{0}$ space is Hausdorff and regular.

Proposition 1.29. Let $R$ be a ring and $B$ a space of prime ideals. Let $I \in B$. Then the following statements are equivalent:

(1) $I=\tilde{I}$.

(2) For all $a \in R$ the statements $a \in I$ and $a_{B}^{\perp} \nsubseteq I$ are equivalent.

(2') For all $a \in R$ the statements $I \in S(a)$ and $I \in \overline{S(a)}$ are equivalent.

(3) Every $h(a), a \in I$ is a neighborhood of $I$ in $B$.

These conditions imply

(4) $I$ is a minimal element in $\bar{B}$.

The space $\{I \in B \mid I=\tilde{I}\}$ is a zero dimensional Hausdorff space.

Proof. For the proof we write $A^{\perp}$ in place of $A_{B}^{\perp}$.

(1) $\Rightarrow(2)$ : Let $a \in I$, then $a \in I$ by (1); thus there is a $b \notin I$ such that $a \in b^{\perp}$. This implies $b \in b^{\perp \perp} \subseteq a^{\perp}$ and therefore $a^{\perp} \nsubseteq I$; since $I$ is prime the implication $a^{\perp} \nsubseteq I \Rightarrow(a) \leqq I$ is trivial. Hence (2) is established. (2) $\Rightarrow(1)$ : Let $a \in I$. By (2) this implies $a^{\perp} \nsubseteq I$. Hence there is an element $b \in a^{\perp}$ with $b \notin I$. But then $a \in a^{\perp \perp} \subseteq b^{\perp} \subseteq I$ (because of $\left.b \notin I\right)$. Hence (1). Since $I \in S(a)$ is equivalent to $a \notin I$ and (in view of $\bigcap S(a)=a^{\perp}$ by 1.14) $a^{\perp} \subseteq I$ is equivalent to $I \in \overline{S(a)},(2)$ is equivalent to $\left(2^{\prime}\right)$. Finally, (3) is equivalent to

For all $a \in I$ we have $I \in$ interior of $h(a)$.

But $I \in$ interior of $h(a)$ is equivalent to $I \notin \overline{S(a)}$ and $a \in I$ is equivalent to $I \notin S(a)$. Therefore $\left(2^{\prime}\right)$ and $\left(3^{\prime}\right)$ are equivalent.

If (1) is satisfied and $J \subseteq I, J \in \bar{B}$, then $\tilde{I} \subseteq \tilde{J} \subseteq J \subseteq I=\tilde{I}$ implies $J=I$. Hence (4).

By $\left(2^{\prime}\right)$ the intersection of the subbasic set $S(a)$ with $\{I \in B \mid I=\tilde{I}\}$ is open closed from which the last assertion follows.

Proposition 1.30. Let $R$ be a ring and $B$ be a space of prime ideals, containing $\operatorname{Max} R$. Then the following statements are equivalent:

(a) $I=\hat{I}$ for all $I \in B$.

(b) All $S(a), a \in R$ are (open and) closed.

(c) $B$ is a zero dimensional Hausdorff space, $B=\operatorname{Max} R$, and $\mathscr{R}$ is a sheaf of simple rings with a Hausdorff sheaf space.

(d) $\mathscr{R}$ is a sheaf of simple rings.

If every proper ideal of $R$ is contained in some maximal proper ideal, then (b) implies

(b') B is a locally compact totally disconnected Hausdorff space (in fact all $S(a)$ are compact open).

Proof. The equivalence of (a) and (b) was shown in 1.29 and (b) immediately implies that $B$ is zero dimensional Hausdorff and therefore 
$B=\operatorname{Max} R$; then 1.27 shows that $\mathscr{R}$ is a sheaf of local rings which, because of (a), must be simple. Let $x, y$ be two points in the sheaf space $E$ of $\mathscr{R}$. If $\rho(x) \neq \rho(y)$ with the sheaf projection $\rho: E \rightarrow B$ of $\mathscr{R}$, then $x$ and $y$ can be separated since $B$ is Hausdorff. If $\rho(x)=\rho(y)$ and $x$ and $y$ cannot be separated, then $z=x-y$ cannot be separated from $0(I), I=\rho(x)$.

The set of all $u \in E_{l}$ which cannot be separated from $O(I)$ forms an ideal of $E_{I}$; since $1(I)$ can be separated from $O(I)$ (indeed $1(B)$ and $O(B)$ are open disjoint neighborhoods of $1(I)$ and $O(I)$, respectively) this ideal is proper and thus must be zero since $E_{I}$ is simple. Hence $z=0(I)$ and $x=y$. Thus $E$ is Hausdorff and (c) is proved.

But (c) trivially implies (d) and (d) implies (a). Let now $I \in B$ and take $a \notin I$. Suppose that $(a)+\bigcap S(a)$ is a proper ideal. If it is contained in some $M \in B$, then $M \in h(a) \cap \overline{S(a)}=h(a) \cap S(a)=\varnothing$ which is impossible. Hence $(a)+a_{B}^{\perp}=(a)+\bigcap S(a)=R$, and thus $S(a)$ is a compact neighborhood of $I$ by 1.9 .

Under the condition (b) of 1.30 , we have $\mathscr{R}(B)=\Gamma(\rho, B)=\Gamma(\rho, S(a))$ $\oplus \Gamma(\rho, h(a))$ for every $a \in R$. We may identify $\Gamma(\rho, X)$ for an open closed $X \subseteq B$ with the subring of $\Gamma(\rho, B)$ of all sections vanishing outside $X$. If there is an identity $e$ of $R$ modulo $\bigcap S(a)=a_{B}^{\perp}$, then $\Gamma(\rho, S(a))$ is contained in the image $\widetilde{R}$ of $R$ in $\Gamma(\rho, B)$ by 1.15 ; hence $\tilde{R}$ is the direct ideal sum of $\Gamma(\rho, S(a))$ and $\tilde{R} \cap \Gamma(\rho, h(a))$. Moreover, $\rho \mid S(a)$ is a sheaf of simple rings with identity; we may assume that $\tilde{e}$ is the characteristic function of $S(a)$ (i.e. takes the value 1 on $S(a)$ and 0 on $h(a)$ ). Then $e$ is a central idempotent of $R(a)=(e)=\Gamma(\rho, S(a))$ (indeed $(a) \subseteq(e)$ is clear, and $(a)$ is not contained in any of the maximal ideals $I e, I \in S(a)$ of $(e))$.

A ring is called biregular if every principal ideal is generated by a central idempotent. Note that a commutative ring is biregular iff it is von Neumann regular. We thus have the following proposition, a portion of which is due to Dauns and Hofmann [19]:

Proposition 1.31. Let $R$ be a ring and $B$ a space of proper prime ideals. If $R$ has identities modulo $a_{B}^{\perp}$ for all $a \in R$ (which is trivially satisfied if $R$ has an identity) then the conditions (a)-(d) of 1.30 are equivalent to

(e) $R / \bigcap B$ is biregular, moreover

$\left(b^{\prime}\right)$ is satisfied.

Proof. We just saw that 1.30 (b) implies (e); for the converse we may assume that $\bigcap B=(0)$. Let $a \in R$ and let $e$ be a central idempotent with $(a)=(e)$. Then for $I \in B$ we have $a \in I$ iff $e \in I$ and thus $S(a)=S(e)$; since $R=R e \oplus R(1-e)$ we have $e \notin I$ iff $I \in R(1-e)$ for every proper prime ideal $I$; therefore $S(e)=h(R(1-e))$, hence $S(a)=S(e)$ is closed. Further $S(e)$ is compact by 1.9 . 
REMARK . In the light of our previous discussion a more general definition of biregularity would be the following: A ring is biregular if every proper ideal is contained in a maximal proper ideal and every principal ideal is a direct factor (i.e. $(a) \oplus a^{\perp}=R$ for all $a \in R$ ). Then a ring is biregular iff it is isomorphic to the ring of global sections with compact support in a sheaf of simple rings over a locally compact zero dimensional space.

About minimal primes. We discussed the case that for all prime ideals in $B$ we have the relation $\tilde{I}=I$. Since the assignment $I \mapsto \tilde{I}$ is fundamental for the whole theory of sectional representation, we give some additional conditions which make (4) equivalent to (1) in 1.29. Most of what follows is due to KeIMEL [53].

Let $R$ be a ring and $B$ a space of proper prime ideals. We recall that for wach subset $A \leqq R$ the ideal $A_{B}^{\perp}$ was the smallest ideal $J$ with $A J+J A$ $\subseteq \bigcup B$ and was given by $A_{B}^{\perp}=\bigcap S(A)$ (1.14). For this discussion we fix $B$ and abbreviate $A_{B}^{\perp}$ by $A^{\perp}$; we also write $a^{\perp}=a_{B}^{\perp}=(a)_{B}^{\perp}$. For an arbitrary prime ideal $P$ (whether contained in $B$ or not) we may define $\widetilde{P}=\bigcup_{a \notin P} a^{\perp}$ : since $P$ is prime, if $P \in \bar{B}=h(\bigcap B)$, the relation $(a) a^{\perp} \subseteq \bigcap B \subseteq P$ and (a) $\nsubseteq P$ implies $a \in a^{\perp} \subseteq P$, hence still $\widetilde{P} \subseteq P$ even then. We will restrict our attention only to prime ideals in $\bar{B}$; we may therefore assume that $\cap B=0$ and $\bar{B}=\operatorname{Spec} R$. Then $R$ is semiprime; therefore $I J=(0)$ and $I \cap J=(0)$ are equivalent for any ideals $I, J$, since $(I \cap J)^{2} \subseteq I J$. Hence $I^{\perp}$ is the largest ideal of $R$ with $I \cap I^{\prime}=(0)$. Let $\mathscr{S}$ be the set of all ideals $I$ of $R$ which are of the form $I=a^{\perp \perp}$ for some $a \in R$. Suppose now that the following condition, due to KEIMEL, is satisfied

(K) For all $a, b \in R$ there is a $c \in R \cup\{1\}$ such that $a^{\perp \perp} \cap b^{\perp \perp}=(a c b)^{\perp \perp}$ (with $a 1 b=a b$ ).

Then $\mathscr{S}$ is a $\cap$-semilattice with zero.

Let $\mathscr{F}$ be an arbitrary filter in $\mathscr{\mathscr { S }}$. We write $P=\bigcup(\mathscr{P} \backslash \overline{\mathscr{F}})$. Then

$$
a \in P \quad \text { iff } \quad a^{\perp \perp} \notin \mathscr{F} .
$$

ProOF. The condition $a \in \bigcup(\mathscr{T} \backslash \mathscr{F})$ is equivalent to the existence of a $b^{\perp \perp} \in \mathscr{S} \backslash \mathscr{F}$ with $a \in b^{\perp \perp}$; but $a \in b^{\perp \perp}$ and $a^{\perp \perp} \subseteq b^{\perp \perp}$ are equivalent because of $a \subseteq a^{\perp \perp}$ and $b^{\perp \perp \perp}=b^{\perp}$, which proves (1). If, in addition, $\mathscr{F}$ is an ultrafilter, then $a^{\perp \perp} \notin \mathscr{F}$ (as in any semilattice with zero) means the existence of a $b \in R$ such that $a^{\perp \perp} \cap b^{\perp \perp}=\{0\}$ and $b^{\perp \perp} \in \mathscr{F}$. We may rewrite $a^{\perp \perp} \cap b^{\perp \perp}=\{0\}$ as $a^{\perp \perp} \subseteq b^{\perp \perp \perp}=b^{\perp}$, and $b^{\perp .1} \in \mathscr{F}$ as $b \notin P$. Since $a \in a^{\perp \perp}$ we have $a \in b^{\perp}$ and thus $P \subseteq \bigcup\left\{b^{\perp} \mid b \notin P\right\}$. Conversely, if $b \notin P$ then $b^{\perp \perp} \in \mathscr{F}$ by (1). Then $b^{\perp} \cap b^{\perp \perp}=b^{\perp} b^{\perp \perp}=0$. Thus $b^{\perp} \notin \mathscr{F}$, and thus

$$
P=\bigcup\left\{b^{\perp} \mid b \notin P\right\} .
$$

Now suppose that $a, b \notin P$. Then $a^{\perp \perp}, b^{\perp \perp} \in \mathscr{F}$ by (1) and therefore $a^{\perp \perp}$ $\cap b^{\perp \perp} \in \mathscr{F}$. By $(\mathrm{K})$ we have an element $c \in R \cup\{1\}$ with $(a c b)^{\perp \perp} \in \mathscr{F}$. Then, 
by (1), acb $\notin P$; since $a^{\perp} a c b=(0)$, we have $a^{\perp} \subseteq(a c b)^{\perp}$, similarly $b^{\perp}$ $\subseteq(a c b)^{\perp}$. Hence $\left\{b^{\perp} \mid b \notin P\right\}$ is an upwards directed family and $P$ is therefore an ideal, and indeed as we have shown, a prime ideal. We can then rewrite (2) as $P=\widetilde{P}$, and by $1.29, P$ is then minimal.

If $I$ is a prime ideal, let $\mathscr{F}_{0}=\left\{a^{\perp \perp} \mid a \notin I\right\}$. Then $(0) \notin \mathscr{F}_{0}$, for if $(0)=\mathrm{a}^{\perp \perp}$ with $a \notin I$, then $a^{\perp} \subseteq I$ since $I$ is prime; but $R=(0)^{\perp}=a^{\perp}$, which is impossible, since $I$ is proper. Moreover, if $a_{k} \notin I$ for $k=1, \ldots, n$, then $a_{k}^{\perp \perp} \nsubseteq I$, hence $a_{1}^{\perp \perp} \cdots a_{n}^{\perp \perp} \nsubseteq I$, which implies $(0) \neq a_{1}^{\perp \perp} \cdots a_{n}^{\perp \perp} \subseteq a_{1}^{\perp \perp} \cap \cdots \cap a_{n}^{\perp \perp}$. Hence $\mathscr{F}_{0}$ is contained in some ultrafilter $\mathscr{F}$ of $\mathscr{S}$. Let $P=\bigcup(\mathscr{S} \backslash \mathscr{F})$. If $a \in P$, then $a^{\perp \perp} \notin \mathscr{F}$ by (1), whence $a^{\perp \perp} \notin \mathscr{F}_{0}$, and this implies $a \in I$. Thus $P \subseteq I$.

We have shown the following result, due to KEIMEL:

Proposition 1.32. Let $R$ be a ring and $B$ a space of prime ideals. Suppose that condition $(\mathrm{K})$ above is satisfied. Then a prime ideal $P$ of $\bar{B}$ is a minimal prime ideal in $\bar{B}$ if and only if $P=\widetilde{P}=\bigcup\left\{a_{B}^{1} \mid a \notin P\right\}$, i.e. (1) $\Leftrightarrow(4)$ in 1.29.

(Note that every $I \in B$ contains some minimal $P \in \bar{B}$ without any hypothesis by Zorn's lemma.)

Let us confirm that there are simple conditions which imply condition (K). Suppose $R$ has the property

(C) For all $a, b \in R$ the relation $a b \in \bigcap B$ implies $b a \in \bigcap B$.

Then $a^{\perp \perp}=\{x \in R \mid u a \in \bigcap B$ implies $u x \in \bigcap B$ for all $u \in R\}$. W. l.g. assume $\bigcap B=(0)$. Now suppose that $x \in a^{\perp \perp} \cap b^{\perp \perp}$, assume $u a b=0$ and try to show that $u x=0$. Since $x \in b^{\perp \perp}$ we conclude that $u a x=0$. Then $a x u=0$ by (C). Thus $x u \in a^{\perp}$ whence $x x u=0$ since $x \in a^{\perp \perp}$. Thus $x u x=0$ by $(C)$ and thus $(u x)^{2}=u x u x=0$. At this point we can conclude $u x=0$ provided we assume

(N) if $a^{n} \in B$ then $a \in \bigcap B$ for all $a \in B$.

We observe that $(\mathrm{N})$ implies $(\mathrm{C})$; indeed $a b \in B$ implies $(b a)^{2} \in b(\bigcap B) a$ $\subseteq \bigcap B$, whence $b a \in \bigcap B$ by $(\mathrm{N})$.

COROLlaRY 1.33. Let $R$ be a ring and $B$ a space of prime ideals. If $a^{n} \in \bigcap B$ implies $a \in \bigcap B$ for all $a \in R$, then a prime ideal $I \in \bar{B}$ is minimal in $\bar{B}$ iff $I=\tilde{I}$, and the subspace of minimal elements in $\bar{B}$ is zero dimensional.

COROLlaRY 1.34. Let $R$ be a commutative ring with identity. Then $I$ is a minimal prime ideal iff $I=\tilde{I}$. The space Min $R$ of minimal primes in $R$ is zero dimensional.

COROllaRy 1.35 (KoH [57]). Let $R$ be a ring without nilpotent elements. Then I is a minimal prime ideal iff $I=\tilde{I}$. The space $\operatorname{Min} R$ is zero dimensional. (In fact, a ring without nilpotent elements is semiprime.)

The simple example of the ring of all sequences $\left(a_{n}\right)_{n \in N}$ of $2 \times 2$ matrices over a field for which there is some natural $m$ (depending on the sequence) 
such that all $a_{n}$ with $n>m$ are diagonal matrices shows that 1.32 fails in general without some condition such as $(\mathrm{K})$.

§2. The Grothendieck construction for commutative rings and its relation to the constructions of $\$ 1$. The first result about sheaf representation of rings is the theorem of Grothendieck in which he establishes the correspondence between commutative rings and affine schemes (1960 [37]). It is embedded into a formidable wealth of material contained in his monumental treatise about algebraic geometry; this may be the reason why it did not enter the field of vision widely when the field of sectional representation began to blossom in the second half of the sixties as a field of interest which is independent of algebraic geometry. It has recently been lifted out of its encyclopedic environment into the more accessible form of a paperback by Macdonald [62]; there it remained exactly in its original form. It is certainly worthwhile to record it here, notably since it has not been possible to my knowledge to completely amalgamate Grothendieck's commutative sectional representation theorem with the general theory discussed in $\S 1$. Our presentation is a trifle different.

Some background. Let, to begin with, $R$ be a commutative ring with identity. We recall that for any multiplicative semigroup $S \leqq R$ which does not contain 0 we can construct the quotient ring $S^{-1} R$ relative to $S$; there is a natural morphism $\lambda_{s}: R \rightarrow S^{-1} R$; every element in $S^{-1} R$ may be represented in the form $\lambda_{s}(r) / \lambda_{s}(s)$ with $r \in R, s \in S$, and the computations are the familiar ones. If $S, T$ are two semigroups with $S \subseteq T, 0 \notin T$, then there is a natural morphism $\lambda_{s}: R \rightarrow S^{-1} R$; every element in $S^{-1} R$ may be repremultiplicative semigroup generated by $A$. If $a, b \in S$, then $\langle a, b\rangle^{-1} R$ $=\langle a b\rangle^{-1} R$; hence $S^{-1} R=\operatorname{colim}\langle a\rangle^{-1} R$, where $a$ ranges through $S$ and the diagram over which we take the colimit is in fact a direct system so that the colimit in question is indeed a direct limit.

Now let $I$ be a prime ideal of $R$. Then $R I$ is a semigroup $S$ which does not contain 0 ; we abbreviate $(R \backslash I)^{-1} R$ by $R_{I}$. Recall that this is a local ring with a unique maximal ideal $\bar{I}=I R_{I}$; by the preceding $R_{I}=\operatorname{colim}\langle a\rangle^{-1} R$, $a \notin I$. In particular, suppose that $\lambda_{s}(r) / \lambda_{s}(a)=0, r \in R, a \notin I$. Then the image of $\lambda_{\langle a\rangle}(r) / \lambda_{\langle a\rangle}(a)$ in $\langle a\rangle^{-1} R$ has to be zero in some $\langle b\rangle^{-1} R$ with $b=a c \notin I$.

The Grothendieck sheaf and the key theorem. Now let $G$ be the disjoint union $\bigcup\left\{R_{I} \times\{I\} \mid I \in B\right\}$ with some $B \subseteq \operatorname{Spec} R$. Define $\gamma: G \rightarrow B$ by $\gamma(x)=I$ iff $x \in R_{I}$. As in $\S 1$, let $S(a)=\{I \in B \mid a \notin I\}$ be the support of $a$ in $B$. In particular $S(1)=B$. Let us briefly write $r / a$ in place of $\lambda_{\langle a\rangle}(r) / \lambda_{\langle a\rangle}(a)$. For any $r / a \in\langle a\rangle^{-1} R$ we have a function $(r / a)^{\wedge}: S(a) \rightarrow G$ given by $(r / a)^{-}(I)$ $=\left(r / a\right.$ in $\left.R_{I}, I\right)$ and $\gamma(r / a)^{\wedge}$ is the inclusion map $S(a) \rightarrow B$. If $(r / a)^{\wedge}(I)=0(I)$ for $I \in S(a)$, then $a$ divides some $b \in R \backslash I$ with $(r / a) \hat{(J)}=0(J)$ for $J \in S(b)$ 
(note $S(b) \subseteq S(a)$ !) by our last observation in the preceding paragraph. Once this is clear we may introduce in $E$ the finest topology making all maps $(r / a)^{\wedge}: S(a) \rightarrow G, r, a \in R, a \neq 0$, continuous. (Basic open sets: $(r / a)^{\wedge}(S(b))$ with $r, a, b \in R, 0 \neq a \mid b$.) After the preceding observations, $\gamma: G \rightarrow B$ is a sheaf $\mathscr{G}$ of commutative local rings with identity, and there is a morphism of rings with identity $r \mapsto \hat{r}: R \rightarrow \Gamma(\gamma, B)$ given by $\hat{r}(I)=\left(r\right.$ in $\left.R_{I} I\right)$. Trivially, this morphism has the kernel $\left\{r \in R \mid r=0\right.$ in $R_{I}$ for all $\left.I \in B\right\}$.

Recall that the kernel of the morphism $\lambda_{s}: R \rightarrow S^{-1} R$ is the set of all $r \in R$ for which there is an $s \in S$ with $r s=0$ (indeed, recall that $S^{-1} R$ is the set of pairs $(r, s) \in R \times S$ modulo the relation $\sim$ with $(r, s) \sim\left(r^{\prime}, s^{\prime}\right)$ iff $r s^{\prime}=s r^{\prime}$ with suitable addition and multiplication; in particular, the image of $r$ in $S^{-1} R$ is the class of $(r, 1)$ consisting of all $(r s, s), s \in S$, and this class agrees with the class $\{0\} \times S$ of $(0,1)$ iff there is an $s \in S$ with $r s=0)$. Thus, with the notation $S^{\perp}=$ largest ideal $J$ with $J S=(0)$, which was used in $\S 1$ we have ker $\lambda_{S}=S^{\perp}$. For a prime ideal $I$ of $R$ an element $\lambda_{R \backslash I}(r)$ is 0 in $R_{I}=$ colim $\langle a\rangle^{-1} R, a \notin I$ iff $\lambda_{\langle a\rangle}(r)$ is zero in $\langle a\rangle^{-1} R$ for $\lambda_{R \mid I}(r)$ some $a \in I$ iff $r a^{n}=0$ for some $a \notin I$ and some natural $n$; in other words, $\operatorname{ker} \lambda_{\langle a\rangle}=a^{\perp}$. We therefore have

2.1.1.

$$
\operatorname{ker} \lambda_{R \backslash I}=\operatorname{ker}\left(R \rightarrow R_{I}\right)=\bigcup_{a \notin I} a^{\perp}=\check{I}
$$

with the notation of $1.23,1.24$.

Therefore, by КoH's result (1.22) we have

$$
\text { 2.1.2. } \quad \text { if } \operatorname{Max} R \cong B \text {, then } \operatorname{ker}(R \rightarrow \Gamma(\gamma, B))=(0) \text {. }
$$

From the relation $\operatorname{ker} \lambda_{\langle a\rangle}=\bigcap\left\{\operatorname{ker} \lambda_{R I I} \mid a \notin I\right\}$, we recover, for the commutative case, Condition 1.24.3: $a^{\perp}=\bigcap\left\{I^{\prime} \mid a \notin I\right\}$; from Theorem 1.23(c), then for $\operatorname{Max} R \cong B \cong \operatorname{Spec} R$ we immediately obtain $R \cong \mathscr{R}^{\prime}$ under $g^{\prime}$. However, with the aid of the sheaf $\mathscr{G}$, more can be said as we shall see in the following discussion.

Let us in fact show the surjectivity of $R \rightarrow \Gamma(\gamma, B)=\mathscr{G}(B)$. Assume that $\sigma \in \Gamma(\gamma, B)$ is global section in this sheaf. In particular $\sigma(I) \in R_{I}$ $=$ colim $\langle a\rangle^{-1} R, a \notin I$; hence $\sigma(I)$ is in the image of some map $\langle a\rangle^{-1} R$ $\rightarrow R_{I}$ for a suitable $a \notin I$; thus $\sigma(I)$ is of the form $r / a^{n}$ in $R_{I}$ (which is our abbreviation for $\left.\lambda_{R \backslash I}(r) / \lambda_{R \mid I}\left(a^{n}\right)\right)$ by the definition of the sheaf topology there is some $b=a c$ such that $\left(r / a^{n}\right)^{\wedge}$ and $\sigma$ agree on $S(b)$; in $\langle b\rangle^{-1} R$ we have $r c^{n} / b^{n}=r / a^{n}$. By renaming, if necessary, we may therefore assume that $\left(r / a^{n}\right)^{\wedge}$ and $\sigma$ agree on $S(a)$. Now we assume that $B$ is quasi-compact. Then by the usual compactness argument we find elements $r(k), a(k) \in R$, $k=1, \ldots, p$, and natural numbers $n(k), k=1, \ldots, p$, such that the local sections $(r(k) / a(k))^{n(k)^{\wedge}}$ and $\sigma \mid S(a(k))$ agree, and $B$ is contained in the union of the $S(a(k))$. Since all $I \in B$ are prime, we have $a \notin I$ iff $a^{n} \notin I$, hence $S(a)$ $=S\left(a^{n}\right)$ for any natural $n$. Thus $B \subseteq \bigcup\left\{S\left(a(k)^{n(k)+1}\right) \mid k=1, \ldots, n\right\}$. The 
ideal $J=\left(a(1)^{n(1)+1}, \ldots, a(p)^{n(p)+1}\right)$ therefore cannot be contained in any $I \in B$, and if $\operatorname{Max} R \cong B$ we can conclude that $J=R$. In particular we obtain a special partition of identity

$$
1=\sum_{k=1}^{p} t^{\prime}(k) a(k)^{n(k)+1}=\sum_{k=1}^{p} t(k) a(k)^{n(k)}
$$

with suitable elements $t(k) \in R$ and $t(k) \mid a(k)$. Now we define $r=\sum_{k=1}^{p} t(k) r(k)$. Then $(\hat{r}-\sigma)(I)=\sum_{k=1}^{p} \hat{t}(k)\left(\hat{r}(k)-\hat{a}(k)^{n(k)} \sigma\right)(I)$; but if $I \in S\left(a(k)^{n}\right)$ then $\left(\hat{r}(k)-\hat{a}(k)^{n(k)} \sigma\right)(I)=0(I)$ in $R_{I}$ by the choice of $r(k), a(k), n(k)$; however, if $I \notin S\left(a(k)^{n}\right)$ then $a(k) \in I$, hence $t(k) \in I$ because of $t(k) \mid a(k)$, and so $\hat{f}(k)\left(\hat{r}(k)-\hat{a}(k)^{n(k)}\right)(I)=0(I)$; therefore all $p$ summands vanish and $\hat{r}=\sigma$. This shows that the Gelfand morphism $R \rightarrow \Gamma(\gamma, B)$ is surjective.

The set $S(a) \subseteq B$ for some $a \in R, a \neq 0$ may be identified with a subspace of $\operatorname{Spec}\langle a\rangle^{-1} R$ which contains $\operatorname{Max}\langle a\rangle^{-1} R$ if $\operatorname{Max} R \cong B$.

We therefore have the following result.

THEOREM 2.1 (GROTHENDIECK). Let $R$ be a commutative ring with identity, $\operatorname{Max} R \subseteq B \subseteq \operatorname{Spec} R$. Then there is a sheaf $\gamma: G \rightarrow B$, with stalks $G_{I} \cong R_{I}=($ localization of $R$ at $I)=(R \backslash I)^{-1} R$, such that all functions $\hat{r}: B \rightarrow G$ are continuous, where $\hat{r}$ associates with $I \in B$ the image of $r \in R_{I}$ for fixed $r \in R$; and the ring morphism $r \mapsto \hat{r}: R \rightarrow \Gamma(\gamma, B)$ is an isomorphism. For each $a \in R$ the natural map $\langle a\rangle^{-1} R \rightarrow \Gamma(\gamma, S(a))$ is an isomorphism.

A completely analogous result is available for modules.

It is clear from the discussion that the nature of Theorem 2.1 is somewhat different from the type of theorem given in $1.15,1.16$ or 1.23 , say. In these cases the stalks of the sheaf associated with $R$ were indeed quotient rings of $R$ so that the map from $R$ into a stalk is surjective, whereas, conversely, in the case of 2.1 above, the stalks are ring extensions of quotients of $R$ and the map of $R$ into a stalk is not generally surjective even though it is epic (in the category of commutative rings with identity). Grothendieck (see also [62]) points out that a presheaf may be defined on the basis of a topology $\mathcal{O}(B)$ rather than on $\mathcal{O}(B)$ itself. One may rephrase 2.1 without great difficulty by saying that the presheaf $S(a) \mapsto\langle a\rangle^{-1} R$ of commutative rings with identity is a sheaf; in the case 1.15 and the related cases in $\$ 1$ the assignment $S(a) \mapsto R / a_{B}^{\perp}$ (resp. $S(a) \mapsto R / a^{\perp}$ ) is not in general a sheaf, but at least has no nonzero locally zero sections (which is, in a sense, halfway towards being a sheaf). In either case the surjectivity of the Gelfand morphism is obtained from a partition of identity argument, but the specific complications are of a slightly different type: In $\$ 1$ we use the smallness of the interior of the radical of a certain canonical sheaf of abelian groups $(1.5-1.6)$ and thereby a certain geometric property of the sheaf space constructed in 1.4 (while the algebraic description of the presheaf as $S(a) \mapsto R / a_{B}^{\perp}$ 
was a second step); on the other hand in 2.1 the partition of identity idea hinges directly on the algebraic definition of the sheaf. As it stands, construction 2.1 depends on the commutativity of $R$, since the algebraic localization $R_{I}$ at a prime ideal is not possible in the noncommutative case without special hypotheses, and even more difficulties arise in imitating the crucial construction $\langle a\rangle^{-1} R$ and the relation $R_{I}=\operatorname{colim}\langle a\rangle^{-1} R$, $a \notin I$. The topological localization $R / \bigcup_{a \notin I} \cap S(a)$ does not, by contrast, depend on commutativity, nor does the relation $\bigcap S(a)=a_{B}^{\perp}$. On the other hand, our knowledge of local rings and therefore of the stalks in the sheaf of 2.1 is vast, whereas the stalks in the sheaves of $\$ 1$ are frequently obscure.

$\mathscr{R}^{\prime}$ and $\mathscr{G}$. However, in the commutative case we understand the relation between the two constructions. We can formulate the following

COROLlaRY 2.2. Let $R$ be a commutative ring with identity and $B$ a space of prime ideals with $\operatorname{Max} R \subseteq B$. Let $\mathscr{R}^{\prime}$ be the sheaf of 1.24 with stalks $\mathscr{R}_{\breve{I}}^{\prime}=R / \breve{I}, I=\bigcup_{a \notin I} a^{\perp}, a^{\perp}=$ annihilator of $a$ in $R$ and let $\mathscr{G}$ be the Grothendieck sheaf of 2.1 with stalks $R_{I}$. Then there is an injection $\mathscr{R}^{\prime} \rightarrow \mathscr{G}$ and $r \mapsto \hat{r}: R \rightarrow \mathscr{R}^{\prime}(B)$ is an isomorphism. Let $\mathscr{R}^{\prime \prime}$ be the subsheaf of $\mathscr{R}^{\prime}$ with stalks $\tilde{I} / \check{I}$ (see 1.24$)$ then $B-\operatorname{Rad} R \cong \mathscr{R}^{\prime \prime}(B)$ under the Gelfand morphism (see 1.24).

Proof. Let $\rho^{\prime}: E^{\prime} \rightarrow B$ be the sheaf projection of $\mathscr{R}^{\prime}$ and $\gamma: G \rightarrow B$ the sheaf projection of $\mathscr{G}$. Then by 2.1 .1 we have a natural injective function $\Phi: E^{\prime} \rightarrow G$ such that $\gamma=\rho^{\prime} \Phi$ and that $\Phi \hat{r}=r^{*}$ where $r \mapsto \hat{r}: R \rightarrow \mathscr{R}^{\prime}(B)$, resp. $r \mapsto r^{*}: R \rightarrow \mathscr{G}(B)$, are the respective Gelfand morphisms. Then $\Phi$ is a morphism of sheaves by the definition of the sheaf topologies on $E^{\prime}$ and $G$. By the definition of $\Phi$ via 2.1.1, it preserves the ring operations. The remaining assertions then follow from 2.1 and 1.23 .

By 2.2 we can consider $\mathscr{R}^{\prime}$ in the commutative case as a subsheaf of the Grothendieck sheaf $\mathscr{G}$. It is exactly the subsheaf whose sheaf space is the union of the global sections in the Grothendieck sheaf:

$$
E^{\prime}=\bigcup\{\sigma(B) \mid \sigma \in \mathscr{G}(B)\}=\bigcup\left\{\text { image }\left(R \rightarrow R_{I}\right) \mid I \in B\right\} .
$$

The conclusion $B$ - $\operatorname{Rad} R \cong \mathscr{R}^{\prime \prime}(B)$ emerges here in an indirect fashion. We saw an example following 1.24 which shows that this fails in the simplest noncommutative cases.

Naturally one would like to know when, at least in the commutative case, the construction $\mathscr{R}^{\prime}$ of $\$ 1$ and the Grothendieck construction yield the same result. We observe that in the commutative case, $R / \check{I}$ and $R_{I}$ are isomorphic if and only if $R / \breve{I}$ is a local ring. Therefore $\mathscr{R}^{\prime} \rightarrow \mathscr{G}$ is surjective if and only if $\mathscr{R}^{\prime}$ is a sheaf of local rings. In 1.28 we obtained a characterization of the case that the smaller sheaf $\mathscr{R}$ is a sheaf of local rings. Clearly, if $\mathscr{R}^{\prime}$ is a sheaf of local rings, then $\mathscr{R}$ is a sheaf of local rings. Now assume that $\mathscr{R}$ is a sheaf of local rings. 
Let $I \in B$ and let $M$ be the unique maximal ideal containing $I$. Let $N \neq M$ be a different maximal ideal; then $\tilde{I} \nsubseteq N$ by assumption, hence there is an $a \notin I$ such that $a_{B}^{\perp} \nsubseteq N$. This means that there is a $b \notin N$ with $a b \in \bigcap B$. If all elements in $\bigcap B$ are nilpotent (which is the case if $B=\operatorname{Spec} R$ ) then $a^{n} b^{n}=(a b)^{n}=0$ for some $n$. Thus $\left(a^{n}\right)^{\perp} \nsubseteq N$ since $b^{n} \notin N$; but also $a^{n} \notin I$, hence $\left(a^{n}\right)^{\perp} \subseteq \breve{I}$. Therefore $\check{I} \nsubseteq N$. Hence $I$ is contained in the unique maximal ideal $M$ and $\mathscr{R}^{\prime}$ is a sheaf of local rings. We summarize:

Corollary 2.3. For a commutative ring $R$ with identity and for a space $B$ of prime ideals $B$ with $\operatorname{Max} R \cong B$ consider the following statements:

(1) The morphism $\mathscr{R}^{\prime} \rightarrow \mathscr{G}$ is an isomorphism.

(2) The sheaf $\mathscr{R}^{\prime}$ is a sheaf of local rings.

(3) The sheaf $\mathscr{R}$ is a sheaf of local rings.

(4) The inclusions $i: \operatorname{Max} R \rightarrow B$ is a Hausdorff embedding and there is a continuous retraction $\mu: B \rightarrow \operatorname{Max} R$ which satisfies $I \leqq \mu(I)$ for all $I \in B$ and is the Stone-Čech compactification B (= Hausdorffization of $B$ in this case).

Then $(1) \Leftrightarrow(2) \Rightarrow(3) \Leftrightarrow(4)$. If all elements of $B-\operatorname{Rad} R=\bigcap B$ are nilpotent (which is the case if $B-\operatorname{Rad} R \subseteq(\operatorname{Spec} R)-\operatorname{Rad} R$, in particular if $B-\operatorname{Rad} R=(0)$ or $B=\operatorname{Spec} R$ ), then (4) $\Rightarrow(1)$ (i.e. all four statements are equivalent).

We may interpret this result as saying that the equivalence of our construction $\mathscr{R}^{\prime}$ and the Grothendieck construction is tantamount to a rather special point set topological property of the $B$-spectrum.

It should be pointed out that there are several attempts to approach Grothendieck's construction in the noncommutative case by creating different concepts of the spectrum. Bergman [7] defines what he calls the Epi-Spectrum Epi-Spec $R$ of the ring $R$; its elements are equivalence classes of ring epimorphisms $f: R \rightarrow E$ into epimorphism final rings $E$, i.e. rings such that every epimorphism $E \rightarrow E^{\prime}$ is injective; the latter are exactly the fields in the commutative case. He singles out the sfield spectrum of classes in which the representatives $f: R \rightarrow E$ have a skew field as domain. It is shown that sfield-Spec $R$ is closed in Epi-Spec $R$. Some indications as to a construction of a sheaf over Epi-Spec $R$ are given, but the version of the theory I have seen needs implementation in this respect. Cohn [14] also produces a sheaf of local rings associated with any ring $R$ with identity and obtains a Gelfand homomorphism from $R$ into the ring of its global sections. Its kernel, however, defines a new radical, whose size needs to be specified in every case; and the image under the Gelfand homomorphism does not in general exhaust the ring of global sections; indeed some Gelfand transforms of noninvertible elements will be invertible in the ring of global sections. It is an unsolved question whether or not the Gelfand morphism 
is an epimorphism in the category of rings. The base space of Cohn's sheaf is not a prime ideal space but a suitable substitute thereof. Hochster's work also contributes to the generalization of the prime ideal spectrum of a commutative ring to the noncommutative case [41].

§3. Sectional representation over Boolean spaces, regular and biregular rings. The theory of sectional representation of not necessarily commutative rings as an area of interest outside algebraic geometry originated in the middle sixties with the memoir of R. S. PIERCE [72] about modules over commutative rings and with J. DAUNS and my paper about biregular rings [19]. These authors by accident or otherwise picked a class of rings which was exceptionally suitable for sectional representation. In view of the fact that a commutative regular ring is biregular, the biregular rings form the larger class of rings. They are characterized by an abundance of central idempotents: Recall from 1.31, that a ring is biregular if every principal ideal is in fact generated by a central idempotent. In particular, the classically well-known test category of Boolean rings is a subclass thereof. One particularly simplifying feature in this context is the fact that all prime ideals are maximal modular: $\operatorname{Spec} R=\operatorname{Max} R$ and that any biregular ring is strongly semisimple: $\bigcap \operatorname{Max} R=(0)$. The sheaf $\mathscr{R}^{\prime}$ over Max $R$ thus agrees with the sheaf $\mathscr{R}$. We saw in 1.30 and 1.31 the deeper reasons why biregular rings should emerge early; the full machinery of $\$ 1$ is not at all necessary for the characterization theorem for biregular rings.

Theorem 3.1 (Dauns AND Hofmann [19]). A ring (resp. commutative ring) is biregular (resp. regular) if and only if it is the ring of all sections with compact support in a sheaf of simple rings with identity (resp. fields) over a locally compact zero dimensional Hausdorff space.

DAUNS and HOFMANN also characterized the ring $\mathscr{R}(\operatorname{Max} R)$ of all global sections of the sheaf associated with a biregular ring $R$ as the ring $L(R)$ of left multipliers (i.e. abelian group endomorphisms $f: R \rightarrow R$ with $f(r s)$ $=f(s) r)$. In general $L(R)$ is not biregular. Not all questions concerning the spectrum of $L(R)$ have been resolved; for some results see DAUNS and HofmanN [20, p. $159 \mathrm{ff}$.]. The center of $L(R)$ is the centroid $C(R)$ of $R$ (i.e. the abelian group endomorphisms $f: R \rightarrow R$ with $f(r s)=f(r) s=r f(s)$ ). It is the ring of all global sections of $\mathscr{R}$ taking their values in the centers of the stalks. VRABEC [95] used the centroid to adjoin an identity to a biregular ring $R$ by forming $R+C(R) \subseteq L(R)$ (where $R$ is the obvious identification with a subring of $L(R)$ ) and thus obtaining indeed a biregular ring with identity, the ring of all global sections in $\mathscr{R}$ which take their central values outside compact sets. One has $\operatorname{Spec}(R+C(R))=\beta(\operatorname{Spec} R)$. A similar phenomenon was observed by DAUNS and HOFMANN [20] in a wide class of rings and algebras (including $C^{*}$-algebras) for the primitive spectrum. 
Thus one would generally say that for purposes of spectral theory and sectional representation of rings the adjunction of identity by adding the centroid is the feasible one.

The characterization theorem for biregular rings itself generalizes older results by ARENS and KAPLANSKY about representation of certain biregular rings as function rings [2] which in turn generalizes the classical results of STONE about the representation of Boolean rings. The characterization theorem was used by DaUns and HofMANN [19] to compute the automorphism group of a biregular ring.

Various generalizations of the theory of biregular rings have been found in the last five years. There are essentially two lines of thought for such generalizations. (A) One weakens the condition for biregularity and arrives at rings which are rings of global sections in sheaves of local (rather than simple) rings over Boolean spaces. (B) More generally, one considers algebraic structures $R$ with a large supply of direct decompositions (as they are given, in the case of a biregular ring with identity by any central idempotent $e$ in the form of $R=R e+R(1-e)$ ). In case (A) consider a ring $R$ (for the sake of simplicity with identity) and a space $B$ of prime ideals containing Max $R$. We say that $R$ is weakly biregular if for two different $I, J \in B$ there is a central idempotent $e \in I, e \notin J$ (equivalently, $J \in S(e)$, $I \notin S(e))$ or vice versa. Since $S(e)$ for an idempotent $e$ is open and closed (with complement $S(1-e)$ (see proof of 1.30)) the Hausdorff property of $B$ follows and we conclude $B=\operatorname{Max} R$. By compactness of Max $R$, the collection of all $S(e)$ is in fact a basis for the topology, and so again Max $R$ is a Boolean space. Then the sheaf $\mathscr{R}$ is a sheaf of local rings over a Boolean space by 1.27 . Conversely, if $\mathscr{R}$ is a sheaf of local rings over a Boolean space, then the central idempotents of $\mathscr{R}(\operatorname{Max} R)$ are exactly the global sections taking the value 1 on a compact open set and the value 0 elsewhere. Then clearly $\mathscr{R}(\operatorname{Max} R)=R / \operatorname{Max}-\operatorname{Rad} R$ is weakly biregular. We therefore have the following result which is partially due to Dauns and Hofmann [20] (see also K EIMEL [49]):

Complement 3.2 to $1.29,1.30,1.31$. Let $R$ be a ring with identity and $B$ a space of prime ideals with $\operatorname{Max} R \subseteq B$. Then the following conditions are equivalent:

(a) $B=\operatorname{Max} R$ and $B$ is a Boolean space.

(b) $\mathscr{R}$ is a sheaf of local rings over a Bool'an space.

(c) $R_{\prime}^{\prime} \cap \operatorname{Max} R$ is weakly biregular.

The result maintains for rings without identity with $\operatorname{Max} R$ as the space of maximal modular ideals and a locally compact zero dimensional Hausdorff space. It has been observed by TILLEMAN [90] that the underlying ring $R$ of a von Neumann algebra is weakly biregular and $\operatorname{Max} R$ is a hyperStonean space, in fact is isomorphic to the maximal spectrum of the center 
$Z(R)$ under $M \mapsto M \cap Z(R)$. TELEMAN used the representation of the underlying ring $R$ of arbitrary von Neumann algebras as the ring of global sections $\mathscr{R}(\operatorname{Max} R)$ of the sheaf of local rings $\mathscr{R}$ in order to obtain an algebraic decomposition theory for von Neumann algebras [91]. We will return to the question of $C^{*}$ - and von Neumann algebras when we discuss sectional representation in functional analysis.

The Boolean decomposition principle. The second idea of generalizing biregularity is incredibly general and may be described as follows:

Let us assume that $R$ is some object in a category $\mathscr{A}$. The set $\mathscr{A}(R, R)$ has the structure of a monoid $M$ under composition of morphisms. Let $E M$ be the set of idempotents in this monoid; this set has a partial order defined by $u \leqq v$ iff $u v=v u=u$, and $1=1_{R}$ is the maximal element. If $\mathscr{A}$ is a pointed category, then $0_{R R}: R \rightarrow R$ is the smallest element. Let us assume that $D \subseteq E M$ is a Boolean sublattice, i.e. a subset containing 0 and 1 in which two elements $u$ and $v$ have a least upper bound $u \vee v$ and in which every element $u$ has a unique complement $u^{\perp}$. In the case of an object $R$ in the category $\mathscr{A}$ of rings with identity the lattice of all central idempotents is isomorphic to such a sublattice under the map which associates with an idempotent $u$ in the center of $R$ the endomorphism $r \mapsto u r$. Let us assumc that we have a set based category in which it makes sense to speak of the image $u R$ for $u \in D$. Let $B$ be the Stone space of the Boolean lattice $D$ : we then have a natural isomorphism $u \mapsto U: D \rightarrow \mathcal{O}_{c}(B) \subseteq \mathcal{O}(B)$ from $D$ onto the lattice of open closed sets in $B$. The assignment $U \mapsto u R: \mathcal{O}_{c}(B)^{\mathrm{op}} \rightarrow \mathscr{A}$ is then a presheaf in the category $\mathscr{A}$ which is defined on the basis $\mathcal{O}_{c}(B)$ of $\mathcal{C}^{C}(B)$; if $F: C^{\prime}(B)^{\text {op }} \rightarrow \mathscr{A}$ is a presheaf from a basis $\mathcal{O}^{\prime}(B)$ of a topology $\mathcal{O}(B)$ into a complete category, one obtains a presheaf through $F U$ $=\lim _{V \in \mathcal{O}^{\prime}(B), V \leqq U} F V$ for $U \notin \mathcal{O}^{\prime}(B)$ (see [37]). A presheaf $P:\left(C_{c}(B)^{\text {op }} \rightarrow\right.$ Set of sets satisfies $P U=\lim _{V \in \mathcal{G}_{c}(B), V \leqq U} P V$ for $U \in \mathcal{O}_{c}(B)$ iff for each finite disjoint decomposition $U=V_{1} \cup \cdots \cup V_{n}$ the map $P U \rightarrow \prod P V_{k}$ is an isomorphism. One can then show that the extended presheaf $P: \mathcal{O}_{c}(B)^{\text {op }} \rightarrow$ Set is in fact a sheaf. Thus $U \mapsto u R$ will be a sheaf iff for any disjoint collection $v_{1}, \ldots, v_{n}$ in $D$ the morphism $u R \rightarrow \prod v_{k} R$ with $u=v_{1} \vee \cdots \vee v_{n}$ is an isomorphism. In view of finite induction this is the case iff $u v=0$ always implies that $(u \vee v) R \rightarrow u R \times v R$ is an isomorphism.

Proposition 3.3 (Boolean decomposition principle). Let $\mathscr{A}$ be a complete pointed category with a faithful, limit preserving set functor. Suppose that each morphism $u: R \rightarrow S$ has a factorization $R \rightarrow u R \rightarrow S$ which under the set functor yields the surjective-injective factorization of a function. Let $R$ be an object and $\mathscr{A}(R, R)$ the monoid with zero of endomorphisms of $R$. Let $D$ be a Boolean lattice in $\mathscr{A}(R, R)$ whose meet operation is composition and whose join is the l.u.b. with respect to the partial order of idempotents in a semigroup. Suppose that for $u, v \in D$ with $u v=0$ the morphism 
$(u \vee v) R \rightarrow u R \times v R$ is an isomorphism. Then there is a sheaf $\mathscr{B}$ over the Boolean spectrum $B$ of $D$ with $R \cong \mathscr{B}(B)$; if $u \in D$ corresponds to the compact open set $U$ on $B$ via the Stone duality then $u R \cong \mathscr{B}(U)$. The stalk $\mathscr{B}_{x}, x \in B$, where $x$ is a prime, hence maximal ideal of $D$, is $\operatorname{colim}_{u \notin x} u R$.

Pierce's Boolean decomposition theorem. Let us test this principle in the case of rings. If $R$ is a ring with identity, let $E(R)$ be the Boolean ring of central idempotents with the addition $(e, f) \mapsto e+f-2 e f$, and with the induced multiplication. Naturally, $E(R)$ also has the structure of a Boolean lattice with $(e, f) \mapsto e+f-e f$ as meet and multiplication as join. For $e \in E(R)$ the subspace $S(e)=\{I \in \operatorname{Spec} R \mid e \notin I\}$ of the spectrum of $R$ is exactly $h((1-e) R)$ and is, therefore, open and closed, and indeed the function $e \mapsto S(e)$ is a lattice injection of $E(R)$ into the Boolean lattice ( ' (Spec $R)$ of all open closed subsets of $\operatorname{Spec} R$. If $R$ is commutative or semiprime, then the representation Theorems 1.17 and 2.1 show that in the canonical sheaves over $\operatorname{Spec} R$ the characteristic functions of any open closed set arise from a central idempotent in the ring. Thus $e \mapsto S(e): E(R)$ $\rightarrow \mathcal{O}_{c}(\operatorname{Spec} R)$ is in fact an isomorphism in this case. (Later we will see that in the case of $l$-rings we have an analogue (see 4.12).)

If $I \in \operatorname{Spec} R$, then $I \cap E(R) \in \operatorname{Spec} E(R)$, and if $M \in \operatorname{Spec} E(R)$, then there is at least one maximal ideal $I \in \operatorname{Spec} R$ with $M \subseteq I$ for which then necessarily $I \cap E(R)=M$. Thus there is a surjective function $\phi: \operatorname{Spec} R$ $\rightarrow$ Spec $E(R)$ given by $\phi(I)=I \cap E(R)$; it is easily checked to be continuous and a quotient map. We point out that for any topological space $Y$ there is a universal "Booleanization" $Y \rightarrow b(Y)$, i.e. a map into a (compact) Boolean space $b(Y)$ such that every continuous map $Y \rightarrow X$ into a Boolean space $X$ factors uniquely through $b(Y)$. One can check that the Booleanization, which is clearly unique up to within natural isomorphism, is given by $Y \rightarrow \operatorname{Spec} \mathcal{O}_{c}(Y), y \mapsto\left\{U \in \mathcal{O}_{c}(Y) \mid y \notin U\right\}$. It turns out to be a quotient map which lumps together exactly the quasi-components of $Y$; a quasicomponent of $Y$ is the intersection of all open closed sets which it meets. We may therefore state that $\phi: \operatorname{Spec} R \rightarrow \operatorname{Spec} E(R)$ is the Booleanization of Spec $R$ if $R$ is commutative or semiprime.

If $e \in E(R)$, then $(1-e) R=e^{\perp}$ in the terminology of 1.24. According to 3.3 we associate with $R$ and $E(R)$ (as a Boolean lattice of endomorphisms $r \mapsto e r, e \in E(R)$, of $R)$ a sheaf $\mathscr{P}$ of rings with identity over $\operatorname{Spec} E(R)$ with $R \cong \mathscr{P}(\operatorname{Spec} E(R))$; the stalks $\mathscr{P}_{x}$ are given by colim ${ }_{e \notin x} e R$; from the exact sequence $0 \rightarrow e^{\perp} \rightarrow R \rightarrow e R \rightarrow 0$ we deduce $\mathscr{P}_{x} \cong R /\left(\bigcup_{\ell \notin x} e^{\perp}\right)$. Hence we have a morphism

$$
\psi_{I}: \mathscr{P}_{\phi(I)} \rightarrow R / \check{I}=\mathscr{R}_{I}^{\prime}, \quad I \in \operatorname{Spec} R,
$$

with $\mathscr{R}^{\prime}$ as in 1.24 , since $\bigcup\left\{e^{\perp} \mid e \notin I, e \in E(R)\right\} \subseteq \bigcup\left\{a^{\perp} \mid a \notin I\right\}=\check{I}$. We observe for the first time in this discussion that we need the concept of a 
morphism of sheaves. Indeed if $\mathscr{A}, \mathscr{B}$ with sheaf projection $\pi: E \rightarrow X$ and $\rho: F \rightarrow Y$ are sheaves (of sets) and $f: Y \rightarrow X$ is a continuous map we can form the pullback sheaf $p: E \times_{f} Y \rightarrow Y, E \times_{f} Y=\left\{(z, y) \in E \times \times_{f} Y \mid \pi(z)\right.$ $=f(y)\}, p(z, y)=y$. We denote the map $(z, y) \mapsto z: E \times_{f} Y \rightarrow E$ by $f^{\prime}$. A morphism $\mathscr{A} \rightarrow \mathscr{B}$ is a pair $(f, \alpha)$ with a continuous function $\alpha: E \times_{f} Y \rightarrow F$ with $\rho \alpha=\rho$. We can describe $(f, \alpha)$ by giving $f$ and a family $\alpha_{y}: \mathscr{A}_{f(y)} \rightarrow \mathscr{B}_{y}$ of maps such that $\alpha: E \times{ }_{f} Y \rightarrow F$ with $\alpha(z, y)=\alpha_{y}(z)$ is continuous. (Caution: many writers call such a morphism a cohomomorphism and write the arrow in the direction in which the arrow $f^{4}$ runs!) It is readily checked that the family $\psi_{I}$ defines a morphism $\mathscr{P} \rightarrow \mathscr{R}^{\prime}$.

In all we record the following result whose essential part is due to PIERCE and which we formulate with some complements:

THEOREM 3.4 (PIERCE's Boolean decomposition theorem for rings). Let $R$ be a ring with identity and $E(R)$ the Boolean algebra (lattice) of central idempotents of $R$. Then there is a sheaf of rings $\mathscr{P}$ over Spec $E(R)$ such that the following conclusions hold.

(a) The stalk $\mathscr{P}_{x}$ is given by $R / \tilde{x}$ where $\tilde{x}=\bigcup\left\{e^{\perp} \mid e \in E(R), e \notin x\right\}$, and $E\left(\mathscr{P}_{x}\right)=\{0,1\}$.

(b) The Gelfand morphism $R \rightarrow \mathscr{P}(\operatorname{Spec} E(R))$ is an isomorphism.

(c) The map $\phi: \operatorname{Spec} R \rightarrow \operatorname{Spec} E(R)$ with $\phi(I)=I \cap E(R)$ is a quotient map; it is the Booleanization of Spec $R$ provided $R$ is commutative or semiprime.

(d) If $I \in \operatorname{Spec} R$, then $\phi(I)^{\sim}=\bigcup\{J \mid J$ is a direct factor of $R$ and $J \subseteq I\}$ and the family $\psi_{I}: \mathscr{P}_{\phi(I)} \rightarrow \mathscr{R}_{I}^{\prime}=R / I$ (induced by the inclusion $\phi(I)^{\sim} \subseteq \check{I}$ ) give a morphism $\psi: \mathscr{P} \rightarrow \mathscr{R}^{\prime}$ with $\mathscr{R}^{\prime}$ as in 1.24.

The Gelfand morphism maps $E(R)$ isomorphically onto the Boolean ring of characteristic functions in $\mathscr{P}(\operatorname{Spec}(E(R)))($ i.e. the continuous sections taking only the values 0 and 1).

In the absence of an identity in $R$ the result maintains, with the modification that the image of the Gelfand morphism in (a) is the ring of global sections with compact support. These results specialize to the case of commutative rings, where $E(R)$ is the Boolean algebra of all idempotents, and to the biregular and weakly biregular situation.

Many authors have utilized sectional representation over Boolean spaces along these lines.

Bergman [4] uses PIERCE's sectional representation to characterize commutative hereditary rings.

We need the following definitions: A projective $R$-module is called hereditarily projective if every one of its homomorphic images in some finitely generated projective module is projective. The ring $R$ is (semi) hereditary if all of its (finitely generated) projective modules are hereditarily projective. A hereditary (resp. semihereditary) commutative integral 
domain is a DEDEKIND (resp. PrüFER) domain. A projective module $P$ over $R$ is weakly hereditarily projective if for every finite set $A \subseteq P$ and every finite set $F \in \operatorname{Hom}_{R}(P, R)$ there is a decomposition $P=P_{1} \oplus P_{2}$ with $A \leqq P_{1}$ and $F\left(P_{2}\right)=(0)$, and if every direct summand of $P$ has the same property; $R$ is called weakly 1-hereditary if $R$ is weakly hereditarily projective over itself and weakly semihereditary if every free module of finite rank is weakly hereditarily projective. Now we have

THEOREM 3.5 (BERGMAN). Let $R$ be a commutative ring with identity. Then $R$ is weakly 1-hereditary iff all stalks $\mathscr{P}_{x}$ in the Pierce sheaf are integral domains. It is weakly semihereditary iff every stalk $\mathscr{P}_{x}$ is a Prüfer domain. It is semihereditary if and only if it is weakly semihereditary and every principal ideal is projective. Finally $R$ is hereditary if and only if every stalk $\mathscr{P}_{x}$ is a Dedekind domain, if every principal ideal is projective, and if the following two conditions hold: (i) The Gelfand transform $\hat{r}$ of a non-zero-divisor takes invertible values outside a finite set. (ii) $E(R)$ is a hereditary Boolean ring.

The injectivity of modules again relates to properties of the Pierce sheaf. KAPLANSKY showed that for a commutative ring $R$ with identity, every simple module is injective if and only if $R$ is von Neumann regular (in which case the sheaves $\mathscr{R}, \mathscr{R}^{\prime}, \mathscr{G}, \mathscr{P}$ all agree and are sheaves of fields). This fails in the noncommutative case; however, the following still maintains (and is proved with the aid of sectional representation $[\mathbf{6 6}])$ :

Proposition 3.6 (MichleR AND Villamayor). Let $R$ be an affine ring (i.e. a ring which is finitely generated as an algebra over its center $Z(R)$ such that each quotient ring satisfies a polynomial identity over $Z(R))$. Then every simple (one-sided) module is injective iff $R$ is von Neumann regular iff $R$ is biregular. In particular 1.30 and 1.31 apply.

Further applications of Boolean decomposition. KeIMEL introduced the ideas around the Boolean decomposition principle 3.3 for semigroups [49] and observed that they worked in the same fashion for universal algebras. His starting point is what he calls a decomposition lattice of a semigroup $S$, namely a family of direct factors of a semigroup which form a Boolean lattice. He constructs the sheaf $\mathscr{B}$ and obtains a sectional representation theorem of the type of 3.3. He continues to investigate in the case of semigroups to what extent one can in fact produce a modified sheaf with nondegenerate stalks. As applications he recovers the most general version of the representation theorem for weakly biregular rings (without identity) and a representation theorem for biregular semigroups. He returns to this idea by proving a representation theorem for $f$-rings which is an analogue of PIERCE's Theorem 3.4. We will discuss this in $\$ 4$.

The starting point of COMER's approach [16] is slightly different, although 
the principal idea is the same. His approach is particularly suitable to the case that the category $\mathscr{A}$ is a category of universal algebras. In this case it is advantageous to start with the concept of a congruence and single out the so-called factor congruences $\phi$ on $R$ which are characterized by the existence of a congruence $\phi^{\prime}$ such that $\phi \cap \phi^{\prime}=1_{R}$ and that the smallest congruence generated by $\phi$ and $\phi^{\prime}$ is $R \times R$. COMER considers such universal algebras for which the set of all factor congruences is a Boolean sublattice of the lattice of all congruences. He then produces a sheaf over the maximal spectrum of this Boolean lattice; this construction can be given geometrically as COMER does it or functorially as in 3.3. The algebra $R$ then emerges as algebra of global sections in a sheaf of algebras of the same type over a Boolean space. If every congruence relation of $R$ which is generated by a proper Boolean ideal of the Boolean lattice of all factor congruences is a proper congruence, then no stalk in the sheaf is degenerate and vice versa. The sheaves which arise are called reduced since the factor congruences of the algebra of global sections arise from open closed decompositions of the base space. COMER also proves the converse which exists for all results in this section: The algebra of global sections in a reduced sheaf satisfies the original hypotheses and the canonical sheaf constructed from it is isomorphic to the given reduced sheaf. COMER's approach applies to all rings with identity; the strength of the result for rings with identity of course depends on the supply of factor congruences; again the class of weakly biregular rings is the only comprehensive one for which enough factor congruences are available in order to obtain sheaf representations over prime ideal spaces. On the other hand, COMER's method was applied by himself to the category of cylindrical algebras. He establishes a duality theory for cylindrical algebras in analogy to the duality of commutative rings and ringed spaces in algebraic geometry; at the same time he furnishes the applications to algebraic logic which are afforded by the sectional representation of cylindrical algebras.

The sectional representation over Boolean spaces, as PIERCE's technique has shown from the beginning, pertains in particular to commutative rings which are generated by their idempotents, and to modules over such rings. These objects are the topic of papers by KeIMEL [51] and BerGMAN [5].

$B a e r$ rings. Sheaf representation of rings over Boolean spaces is also very suitable for the class of Baer rings. A ring $R$ with identity is a Baer ring if the annihilator ideal $a^{\perp}$ of every element is generated by a central idempotent. Such a ring is semisimple since it cannot contain nilpotent principal ideals. Let $\mathscr{P}$ be its Pierce sheaf. Then all stalks $\mathscr{P}_{x}$ are domains; for let $a, b \in \mathscr{P}(B)$ with $a(x) \neq 0$ but $a(x) b(x)=0$, then there is a compact open neighborhood $U$ of $x$ such that $a(y) b(y)=0$ for $y \leqslant U$. Let $e \in \mathscr{P}(B)$ be the characteristic function of $U$. By replacing $a$ and $b$ by $a e$ and $b e$, respectively, we may 
assume that $a b=0$; if $f$ is the central idempotent generating the annihilator of $a$ then $b f=b$; now $f(x)$ must be either 0 or 1 ; since $a(x) f(x)=0$ and $a(x) \neq 0$ we must have $f(x)=0$ and thus $b(x)=(b f)(x)=0$. Conversely, if $\mathscr{P}$ is a sheaf of domains over a zero dimensional Hausdorff space $B$, then $\mathscr{P}(B)$ is a Baer ring if and only if the sheaf is Hausdorff (PEERCY [71]); indeed Hausdorffness of the sheaf space of any sheaf means that the subspace on which two sections agree is open closed in the intersection of their domains. Now if every $a \in \mathscr{P}(B)$ has open closed support then clearly $\mathscr{P}(B)$ is a Baer ring; conversely, if $\mathscr{P}(B)$ is a Baer ring and $a \in \mathscr{P}(B)$, then $a^{\perp}$ is the ideal of all sections $b$ whose support is contained in the open zero set of $a$; since $a^{\perp}$ is generated by a central idempotent, i.e. a characteristic function $e$, we conclude that the open zero set of $a$ is the support of $e$ and is, consequently also closed. One thus can summarize :

Proposition 3.7. Let $R$ be a ring with identity. Then $R$ is a Baer ring if and only if its Pierce sheaf is a Hausdorff sheaf of (not necessarily commutative) domains over a Boolean space.

The ideal $I_{x}$ of all sections vanishing at a point $x$ is clearly the kernel of $R \rightarrow \mathscr{P}_{x}$ and is, therefore, prime. After the preceding, any of its elements is annihilated by some element not belonging to it. Thus $I_{x} \subseteq \tilde{I}_{x} \subseteq I_{x}$ which implies that $I_{x}$ is minimal (see 1.29,1.32). On the other hand if $P$ is a prime ideal of $R$, then $E(R) \cap P$ is a prime ideal, hence a maximal ideal of the Boolean ring $E(R)$.

COROLlaRY 3.8. For a Baer ring $R$, let $E(R)$ be its Boolean ring of central idempotents. Then there is a commutative diagram of continuous maps

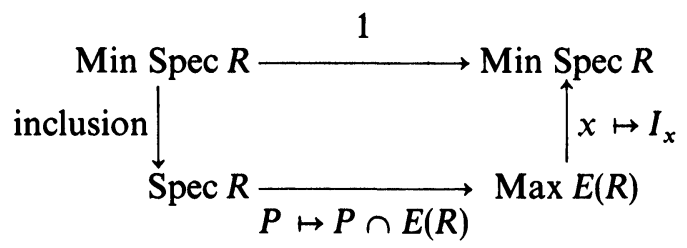

In particular, the injection $x \mapsto I_{x}: \operatorname{Max} E(R) \rightarrow \operatorname{Min} \operatorname{Spec} R$ is an isomorphism (KIST [55]).

The patch topology on Spec $R$. As HochSTER has shown [39] and as was known to GROTHENDIECK, there is another universal way of associating a Boolean space with any commutative ring $R$ with identity. Indeed, on Spec $R$, the quasi-compact sets $S(a)=\{I \in \operatorname{Spec} R \mid a \notin I\}$ and their complements $h(a)$ generate a zero dimensional compact Hausdorff topology on Spec $R$; the Boolean space with this so-called patch topology will be denoted by $\operatorname{Spec}_{p} R$. (The significance of the patch topology for the spectrum of lattices and its general background was investigated in [45].) 
We apply the general construction 1.4 to $B=\operatorname{Spec}_{p} R$ and to the family $\left\{K_{I} \mid I \in \operatorname{Spec} R\right\}, K_{I}=I$. The basic neighborhoods of $I$ in $B$ are given by $U\left(a_{0} ; a_{1}, \ldots, a_{n}\right)=S\left(a_{0}\right) \cap h\left(a_{1}\right) \cap \cdots \cap h\left(a_{n}\right)$ with $a_{0} \notin I, a_{1}, \ldots, a_{n} \in I$. Now $a \in K(U(1 ; a))=\bigcap\{J \in \operatorname{Spec} R \mid 1 \notin J, \quad a \in J\} ;$ hence $I=K_{I}$ $\cong \bigcup_{a \in I} K(U(1 ; a)) \cong \bigcup_{I \in U} K(U)=\widetilde{K}_{I}$, where $U$ ranges through all $B$-neighborhoods of $I$. The canonical construction of $\S 1$ therefore enables us to introduce a topology on $E=\bigcup\{R / I \mid I \in \operatorname{Spec} R\}$ which makes the obvious map $\alpha: E \rightarrow \operatorname{Spec}_{p} R$ a sheaf projection of a sheaf $\mathscr{D}$ of integral domains. The topology is generated by the sets $\hat{r}(\operatorname{Spec} R)$ with $\hat{r}: \operatorname{Spec} R$ $\rightarrow E$ given by $\hat{r}(I)=r+I$. The Gelfand map $r \mapsto \hat{r}: R \rightarrow \Gamma(\alpha)$ is injective, but not in general surjective. We denote by $k_{I}$ the quotient field of $R / I$ and denote by $F$ the disjoint union $\left\{k_{I} \mid I \in \operatorname{Spec} R\right\}$, and let $\beta: F \rightarrow \operatorname{Spec} R$ be the index projection. Set $a \neq 0$; then $\hat{a}(I) \neq 0$ for $I \in S(a)$. In $F$ we can form $(\hat{a} \mid S(a))^{-1}$ and then $(\hat{b} \mid S(a)) /(\hat{a} \mid S(a))^{-1}$ for any $b \in R$. The images of all of these local sections in $F$ generate a topology which makes $\beta: F \rightarrow \operatorname{Spec}_{p} R$ into the projection of a sheaf $\mathscr{F}$ of fields. Then $\mathscr{F}\left(\operatorname{Spec}_{p} R\right)$ is a commutative von Neumann regular ring with identity and $\operatorname{Spec}_{p} R$ is naturally isomorphic to its spectrum $(1.31,3.1)$. Moreover, it contains a copy of $R / \operatorname{Rad} R$, with $\operatorname{Rad} R=\bigcap \operatorname{Spec} R$. This yields a portion of the following result, which is due to WIEGAND [96], with some parallel developments by OLIVIER:

THEOREM 3.9. Let $R$ be a commutative ring with identity, and let $B=\operatorname{Spec}_{p} R$ be the spectrum of $R$ with HOCHSTER's patch topology, which makes it into a Boolean space. Then there is a sheaf of integral domains $\mathscr{D}$ and a sheaf of fields $\mathscr{F}$ over $B$ with $\mathscr{D} \subseteq \mathscr{F}$ such that $\mathscr{D}_{I}=R / I$ and $\mathscr{F}_{I}$ is the quotient field of $R / I$. The map $R \rightarrow \mathscr{D}(B) \rightarrow \mathscr{F}(B)$ is a homomorphism into a regular ring $\operatorname{Reg} R=\mathscr{F}(B)$ with spectrum isomorphic to $B$. The kernel is precisely $\bigcap \operatorname{Spec} R$. The assignment $\mathrm{Reg}$ is a functor from the category of commutative rings with identity into the subcategory of regular commutative rings which is left adjoint to the inclusion functor, i.e. every morphism from $R$ into a regular ring factors uniquely through $\operatorname{Reg} R$.

Since $\mathscr{F}$ as a sheaf of simple rings over a Bòolean space has a Hausdorff sheaf space by 1.30 , then $\mathscr{D}$ has a Hausdorff sheaf space. Then $\mathscr{D}(B)$ is a Baer ring and $\mathscr{D}$ is the PIERCE sheaf of $\mathscr{D}(B)$. After $3.4, \mathscr{D}(B)$ is also weakly 1-hereditary. If $\hat{R}$ denotes the image of $R$ in $\mathscr{D}(B)$ obtained through $r \mapsto \hat{r}$, and if $E(\mathscr{D}(B))$ denotes the Boolean ring of all idempotents, i.e. characteristic functions of $\mathscr{D}(B)$, then it is easy to see that $\mathscr{D}(B)=\hat{R} \cdot E(\mathscr{D}(B))$ (by which we mean the ring of all linear combinations $\sum r_{i} \cdot e_{i}$ with $r_{i} \in R$ and $\left.e_{i} \in E(\mathscr{D}(B))\right)$. In particular every semiprime commutative ring $R$ with identity is contained in a Baer ring $S$ which is generated by $R$ und the idempotents of $S$. Such a super ring $S$ is called a Baer extension of $R$. Thus we have 
COROLlaRY 3.10. The ring $\mathscr{D}\left(\operatorname{Spec}_{p} R\right)$ is a Baer extension for any commutative ring $R$ with identity.

Baer extensions as such, however, can be obtained in a more economical fashion. On the subset $\operatorname{Min} R=\{P \in \operatorname{Spec} R \mid P$ minimal in $\operatorname{Spec} R\}$ the hull-kernel topology and the patch topology agree since $\operatorname{Min} R \cap S(a)$ is open and closed by $1.29,1.32$.

Since every prime ideal is contained in a minimal one, $\bigcap$ Min $R$ $=\bigcap \operatorname{Spec} R$. If we denote by $\mathscr{D}^{\prime}=\mathscr{D} \mid \operatorname{Min} R$ the restriction $\alpha^{-1} \mid \operatorname{Min} R$ : $\alpha^{-1}$ Min $R \rightarrow$ Min $R$, then $\mathscr{D}^{\prime}$ is a sheaf of domains over a zero dimensional Hausdorff space with zero dimensional sheaf space. In particular, $r \mapsto \hat{r} \mid \operatorname{Min} R: R \rightarrow \mathscr{D}^{\prime}(B)$ is a homomorphism of $R$ into a Baer ring with kernel $\operatorname{Rad} R=\bigcap \operatorname{Spec} R$.

KIST [55] has in fact shown the following result :

COROLlaRY 3.11. The subring $R^{\prime}$ of $\mathscr{D}^{\prime}(\mathrm{Min} R)$ generated by the image of $R \rightarrow \mathscr{D}^{\prime}(\operatorname{Min} R)$ and the characteristic functions of $\operatorname{Min} R \cap S(a), a \in R$, is a Baer extension of $R / \bigcap$ Spec $R$. If Min $R$ happens to be compact (which is the case if for any $a \in R$ there is an $x \in R$ with $a^{\perp}=x^{\perp \perp}$ by a result of HENRIKSEN and JERISON [38]) then $R^{\prime}=\mathscr{D}^{\prime}(\mathrm{Min} R)$.

KeIMEL [52] suggests a different process: For a commutative ring $R$ with identity he considers the complete Boolean lattice $L$ of annihilators $A^{\perp}$, $A \subseteq R$. With each maximal ideal $M$ of $L$ he associates the ideal $I(M)$ $=\bigcup\{J \mid J \in M\}$ which he shows to be in $\operatorname{Spec} R$. Then $M \mapsto I(M): \operatorname{Spec} L$ $\rightarrow \operatorname{Spec}_{p} R$ turns out to be a continuous map. The image $X$ of this map contains Min $R$ and is compact. KeIMEL considers the pullback sheaf of $D \mid X$ over Spec $L \rightarrow \operatorname{Spec}_{p} R$ and obtains its ring of sections as a Baer extension. This extension is, in fact, a complete Baer ring in the sense that the annihilator of every subset is generated by a central idempotent.

Independently from KeIMEL, DAVIS [22] has described the same process in more general form by introducing axiomatically an orthogonality relation on $R$ and thereby introducing a complete Boolean lattice in the lattice ideals of $R$. DAvis also gives a characterization of the subspace $X \cong \operatorname{Spec}_{p} R$ : Indeed a prime ideal $I \in \operatorname{Spec} R$ is in $X$ if and only if (1) $a, b \in I$ implies $\left(a^{\perp \perp}+b^{\perp \perp}\right)^{\perp \perp}\left(=1 . u\right.$.b. of $a^{\perp \perp}$ and $b^{\perp \perp}$ in the Boolean lattice of polars) $\subseteq I,(2) a \in I$ implies $a^{\perp} \neq(0)$. DAvis also shows that $X=\operatorname{Min} R$ if and only if for all $a, b \in R$ there are $a^{\prime} \in a^{\perp}, a^{\prime \prime} \in a^{\perp \perp}$ such that $b \in\left(a^{\prime}+a^{\prime \prime}\right)^{\perp \perp}$.

§4. Sectional representation of lattice-ordered structures. In the case of rings we saw that a general representation theory by sections in sheaves is available and observed that it is particularly satisfactory for special classes of rings which have either particular algebraic properties such as commutativity, biregularity, etc. or particular spectral properties such as Haus- 
dorff structure space. There is, however, a wide class of rings which in view of additional structure has a very gratifying sectional representation theory, namely, the class of rings with lattice structure.

Generalities. An excellent source of reference for this material is Keimel's set of Tulane Lecture Notes [54].

Definition 4.1. A lattice-ordered ring (briefly $l$-ring) is a ring $R$ with a lattice order such that for all $a, b, c \in R$ we have

(1) $a \leqq b$ implies $a+c \leqq b+c$,

(2) $a \leqq b$ und $0 \leqq c$ imply $a c \leqq b c$ und $c a \leqq c b$.

Note that this includes the case that all products $a b$ are 0 , in which case (2) is trivially satisfied. Thus the case of lattice ordered abelian groups (i.e. commutative groups with a lattice order satisfying (1)) is included.

The $l$-rings clearly form a category $l$ whose morphisms are ring morphisms and lattice morphisms. The kernel of an $l$-morphism is called an $l$-ideal; they are exactly the ring ideals which are order convex sublattices. One of the fundamental facts, which make the ideal theory of $l$-rings exceptionally pleasant is the following:

THEOREM 4.2. For an l-ring $R$ the set of all l-ideals is a complete distributive lattice relative to intersection and sum.

The place of prime ideals in general ring theory is taken by the irreducible ideals in the case of $l$-rings.

Definition 4.3. An $l$-ideal $I$ is irreducible if for any pair $J, K$ of $l$-ideals $J \cap K \subseteq I$ implies $J \subseteq I$ or $K \cong I$. The space of all irreducible proper $l$-ideals of $R$ with the hull-kernel topology is denoted by $\operatorname{Irr} R$. Every $l$-ideal $I$ of $R$ which is maximal in the collection of all $l$-ideals of $R$ which do not contain a fixed, but arbitrary element $a \in R$ is irreducible.

Proposition 4.4. For every l-ideal $I$ we have $I=\bigcap\{J \in \operatorname{Irr} R \mid I \subseteq J\}$. The hull-kernel topology is generated by the basic sets $S(a)=\{I \in \operatorname{Irr} R \mid a \notin I\}$ as it was in the case of Spec $R$. If we set $S(A)=\{I \in \operatorname{Irr} R \mid A \nsubseteq I\}$ for $A \cong R$, then the function $I \mapsto S(I)$ is a lattice isomorphism from the lattice of all l-ideals of $R$ onto the lattice of open sets of $\operatorname{Irr} R$. (This is reminiscent of the situation of $\operatorname{Spec} R$ for a commutative ring $R$ with identity.) The sets $S(a)$, $a \in R$ are exactly the quasi-compact open subsets of $\operatorname{Irr} R$.

For details about all of these facts we refer to KeImeL [54].

Sheaves of l-rings and Keimel's main theorem.

DEFINITION 4.5. A sheaf of l-rings is a sheaf of rings all of which are $l$-rings and in which the lattice operations are continuous. Clearly, in a sheaf of rings, all full sets of sections over a subset of the base space form an l-ring under pointwise operations. 
For a space $B \subseteq \operatorname{Irr} R$ and $I \in B$ we can define 4.5.1. $\tilde{I}=\bigcup_{I \in U} \bigcap U$, where $U$ ranges through the neighborhoods of $I$
in $B$.

Clearly

$$
\tilde{I}=\bigcup_{a \notin I} \cap S(a) \text {. }
$$

(Compare 1.4.3 and 1.12.2). We also have an analog of KeIMEL's Lemma 1.14 .

LEMMA 4.6. Let $B$ be an arbitrary collection of irreducible l-ideals in an l-ring $R$, let $I$ be an l-ideal and $A$ a subset of $R$. Then the following conditions are equivalent :

(1) $I=\bigcap S(A)$.

(2) $I$ is the largest l-ideal with $(A) \cap I \subseteq \bigcap B$, where $(A)$ is the l-ideal generated by $A$.

The proof is analogous to the proof of 1.14. We again denote $\bigcap S(A)$ with $A_{B}^{\perp}$ and write $a_{B}^{\perp}$ in place of $(a)_{B}^{\perp}$. The general construction 1.4 applied to $R$ and the family $B$ produces a sheaf $\mathscr{R}$ of l-rings, $\rho: E \rightarrow B$ with stalks $E_{I}=R / \tilde{I} \times\{I\} \cong R / \tilde{I}$, where

$$
\tilde{I}=\bigcup_{a \notin I} a_{B}^{\perp}
$$

The Gelfand morphism $r \mapsto \hat{r}: R \rightarrow \mathscr{R}(B)=\Gamma(\rho, B)$ is an l-morphism with kernel $\bigcap B$ by the equivalent of 1.7. There is, however, in the current situation one aspect which goes beyond Theorem 1.15; KelMEL shows that we can find a larger support system $\Phi(1.9)$ such that $\mathscr{R}$ is $\Phi$-soft and that $R$ contains $\Gamma_{\Phi}(\rho, B)$. Indeed KeIMEL proves the following:

LEMMA 4.7. Let $B \subseteq \operatorname{Irr} R$ be a subspace such that each proper l-ideal $I \subseteq R$ is contained in some $J \in B$. Let $\sigma \in \Gamma(\rho, X)$ for $X$ closed and quasicompact in $B$. Then $\sigma=\hat{r} \mid X$ for some $r \in R$. Further, if $\Phi$ denotes the collection of all quasi-compact closed sets, then $\Gamma_{\Phi}(\rho, B) \subseteq R$.

INDICATION OF PROOF (SEE [54] FOR DETAILS). We assume without loss of generality $\sigma \geqq 0$. The method of $\S 1$ gives a finite cover $X \subseteq U_{1} \cup \cdots \cup U_{n}$ by open sets of $B$ and elements $a_{1}, \ldots, a_{n} \in R$ such that $\sigma\left|X \cap U_{k}=\hat{a}_{k}\right| X$ $\cap U_{k}$ for $k=1, \ldots, n$. Let $a_{0}=0, U_{0}=B \backslash X$. Without loss of generality we may assume $a_{k} \geqq 0$. If $I_{k}=\bigcap\left(B \backslash U_{k}\right), k=0, \ldots, n$, then the hypothesis about $B$ implies $R=I_{0}+\cdots+I_{n}$. Let $b=a_{0} \vee \cdots \vee a_{n} \geqq 0$; by a property which can be established for all $l$-rings one can find elements $b_{k} \in I_{k}, 0 \leq b_{k}, k=0, \ldots, n$, such that $b=b_{0} \vee \cdots \vee b_{n}$.

Finally define $a=\left(a_{0} \wedge b_{0}\right) \vee \cdots \vee\left(a_{n} \wedge b_{n}\right)$. Then one can show that 
$(\sigma-\hat{a} \mid X)(X) \subseteq \operatorname{Rad} E \cap \rho^{-1}(X)$, whence $\sigma=\hat{a} \mid X$ by 1.8. If $\sigma$ is a global section with support $X$, then $\hat{a}=\sigma$ if $a$ is constructed as above. Thus one obtains the following theorem:

THEOREM 4.8 (KeIMEL's representation theorem for l-rings). Let $R$ be an l-ring and $B \subseteq \operatorname{Irr} R$ a space of irreducible ideals such that every proper l-ideal of $R$ is contained in some $I \in B$. Let $\Phi$ be the support system of all closed quasi-compact subsets of $B$. Then there is a $\Phi$-soft sheaf of l-rings $\mathscr{R}, \rho: E \rightarrow B$ with stalks $\mathscr{R}_{I}=R / \tilde{I}, \tilde{I}=\bigcup_{a \notin I} a_{B}^{\perp}$, where $a_{B}^{\perp}$ is the maximal l-ideal $J$ with $(a) \cap J=\bigcap B$. There is an exact sequence $0 \rightarrow \bigcap B \rightarrow R$ $\rightarrow \mathscr{R}(B)$, and the image of the last map contains $\Gamma_{\Phi}(\rho, B)$. If $B=\operatorname{Irr} R$, then $\cap B=0$, and $a_{B}^{\perp}=a^{\perp}$.

COROLlary 4.9. If $L$ is an l-ring with a formal unit (i.e. an element which is not contained in any $I \in \operatorname{Irr} R$; an identity is a formal unit), then $R$ is isomorphic to the full l-ring of global sections in a sheaf of l-rings $R / \tilde{I}$ over $\operatorname{Irr} R$. Indeed, for a formal unit $u$ the space $S(u)=\operatorname{Irr} R$ is quasi-compact.

Local l-rings. We will call an $l$-ring $R$ a local $l$-ring (KeIMEL: quasilocal) if it has exactly one proper maximal ideal containing all other proper ideals. The developments of $1.25 \mathrm{ff}$. carry over to the case of $l$-rings and show that $\mathscr{R}$ is a sheaf of local $l$-rings if and only if $\operatorname{Max} \operatorname{Irr} R \rightarrow \operatorname{Irr} R$ is a Hausdorff embedding. (See 1.26.) In this case all ideals $\tilde{I}$ are $I$-primary for $I \in \operatorname{Max} \operatorname{Irr} R$, i.e. if $J \subseteq I$ is such that $R / J$ is a local $l$-ring with unique proper maximal ideal $I / J$, then $\tilde{I} \subseteq J$, and $R / \tilde{I}$ is a local $l$-ring. 1.29 carries over from $\operatorname{Spec} R$ to $\operatorname{Irr} R$ without change.

We also have an analogue of 1.30 :

Proposition 4.10. Let $R$ be an l-ring and $\operatorname{Max} \operatorname{Irr} R \subseteq B \cong \operatorname{Irr} R$. Then the following statements are equivalent:

(a) $I=\tilde{I}$ for all $I \in B$.

(b) All $S(a), a \in R$ are (open and) closed, in fact they are compact.

(c) $B$ is a zero dimensional Hausdorff space, $B=\operatorname{Max} R$, and $\mathscr{R}$ is a sheaf of simple l-rings (i.e. nonsingleton l-rings with proper nonzero l-ideal) with a Hausdorff sheaf space.

(d) $\mathscr{R}$ is a sheaf of simple l-rings.

In any $l$-ring $R$ the principal $l$-ideal $(a)$ generated by $a \in R$ is a direct factor iff $(a)+a^{\perp}=R$. If every principal $l$-ideal in $R$ is a direct factor, we call $R$ a biregular l-ring (KeIMEL: quasi-regular). We then also have

COROLlaRY 4.11. In 4.10 the conditions (a)-(d) are also equivalent to (e) $R / \cap B$ is a biregular l-ring.

Keimel's Boolean decomposition theorem. The analogy between the theory for rings and the theory for $l$-rings can be carried much further. As 
an example, one observes that the special properties of the lattice of $l$-ideals (see 4.2) allow a particularly efficient application of the Boolean decomposition principle 3.3. In fact we have a precise analogue of PIERCE's Theorem 3.5 which can be obtained from 3.3 and which KeIMEL derives from a slightly generalized version of 4.8 . In the case of arbitrary rings $R$ with 1 we had to apply the Boolean decomposition principle to the Boolean lattice $E(R)$ of central idempotents; we could replace $E(R)$ by the Boolean lattice $\mathcal{U}_{c}(\operatorname{Spec} R)$ of open closed sets of Spec $R$ only in the special cases of commutative or semiprime $R$. From Proposition 4.4 it follows, however, that for $l$-rings $\mathcal{O}_{c}(\operatorname{Irr} R)$ is exactly isomorphic to the Boolean lattice of all direct factors of $R$.

THEOREM 4.12 (KeIMEL's decomposition theorem for l-rings). Let $R$ be an l-ring. Then there exists a sheaf $\mathscr{K}$ of l-rings over the maximal ideal space $B=\operatorname{Spec}\left(\mathcal{O}_{c}(\operatorname{Irr} R)\right)$ such that the following conclusions hold:

(a) The stalk $\mathscr{K}_{x}$ is given by $\mathscr{K}_{x}=R / \tilde{x}$, where the l-ideal $\tilde{x}$ is given by $\tilde{x}=\bigcup\left\{\bigcap U \mid U \in \mathcal{O}_{c}(\operatorname{Irr} R), U \notin x\right\}$ and is directly indecomposable, i.e. Irr $\mathscr{R}_{x}$ is connected.

(b) The Gelfand morphism $R \rightarrow \mathscr{K}(B)$ is an isomorphism.

(c) The map $\phi: \operatorname{Irr} R \rightarrow B$ with $\phi(I)=\left\{U \in \mathcal{O}_{c}(\operatorname{Irr} R) \mid I \notin U\right\}$ is a quotient map and is the Booleanization of $\operatorname{Irr} R$ (which collapses exactly the quasicomponents of $\operatorname{Irr} R$ (see discussion preceding 3.4)).

(d) If $I \in \operatorname{Irr} R$, then $\phi(I)^{\mathcal{\sim}}=\bigcup\{J \mid J$ is a direct factor of $R$ and $J \subseteq I\}$ and the family $\psi_{I}: \mathscr{K}_{(I)} \rightarrow \mathscr{R}_{I}=R / \tilde{I}$ defined by the inclusion $\phi(I) \cong \tilde{I}$ give a morphism $\mathscr{K} \rightarrow \mathscr{R}$ with $\mathscr{R}$ as in 4.8 .

f-rings. In the case of arbitrary rings we obtained special results for the subclass of commutative rings. Analogously, within the class of $l$-rings one has a subclass which plays a similar role as the class of commutative rings in the class of all rings, namely the class of $f$-rings.

DEFINITION 4.13. An $l$-ring $R$ is called an $f$-ring if $a \wedge b=0$ implies $a \wedge b c=a \wedge c b=0$ for all $a, b, c \geqq 0$ in $R$.

For our purposes, the following results are the most crucial ones (see Keimel [54]).

Proposition 4.14. If $I$ is a proper l-ideal in an f-ring $R$ then the following conditions are equivalent:

(a) $R / I$ is totally ordered.

(b) $I \in \operatorname{Irr} R$.

(c) (Resp. (c')) $a \wedge b=0$ implies $a \in I$ or $b \in I$ for all $a, b \in R$ (resp., for all $a, b \in R$ with $a, b \geq 0$ ).

Since $\bigcap \operatorname{Irr} R=(0)$, an $l$-ring is then an $f$-ring iff it is a subdirect product of totally ordered rings. For an $f$-ring $R$ and $I \in \operatorname{Irr} R$ the subspace $\{I\}^{-}$ $=\{J \in \operatorname{Irr} R \mid I \cong J\}$ is totally ordered under inclusion. 
Proposition 4.15. If $I$ and $J$ are incomparable elements of $\operatorname{Irr} R$ for an f-ring $R$, then $I$ and $J$ have disjoint open neighborhoods in $\operatorname{Irr} R$.

With reference to $1.26,1.27$ we formulate

Corollary 4.16. For an $f$-ring $R$ the inclusions $\operatorname{Min} \operatorname{Irr} R \rightarrow \operatorname{Irr} R$ and Max Irr $R \rightarrow \operatorname{Irr} R$ of the space of minimal (resp. maximal) irreducible ideals are Hausdorff embeddings.

In particular, for Max Irr $R \subseteq B \cong \operatorname{Irr} R$ the sheaf $\mathscr{R}$ is a sheaf of local $f$-rings. Another feature of commutative rings, namely, that $I=\tilde{I}$ characterizes the minimal prime ideals, yields an analogue for $f$-rings:

Proposition 4.17. The following statements are equivalent for an irreducible ideal I of an f-ring $R$ :

(a) $I=\tilde{I}$.

(b) $a \in I$ iff $a^{\perp} \notin I$ for all $a \in R$.

(c) $I \in \operatorname{Min} \operatorname{Irr} R$.

This result is due to the fact that for principal $l$-ideals in an $f$-ring we have what amounts to condition $(\mathrm{K})$ preceding 1.32 :

Indeed $(a) \cap(b)=(a \wedge b)$ for all $a, b \in R$ with $a, b \geqq 0$. This naturally leads to a sectional representation theory of $f$-rings over its minimal ideal space which is similar to what we commented on in the corresponding context with commutative rings, Baer rings, etc. (see KeIMEL [54, 6.9]); the analogue of the Baer rings are the so-called projectable $f$-rings, i.e. $f$-rings in which every principal polar $a^{\perp}$ is a direct factor. The pertinent sheaf representation theorems are given in KeIMEL [54, 6.12, 6.13]. It should be mentioned that HocHSTER's patch topology on the spectrum of a commutative ring has an analogue for all $f$-rings.

Investigations along these lines have been undertaken by S. J. BERNAU for $l$-groups. In any case, for an $f$-ring $R$, the $\operatorname{space}^{\operatorname{Irr}_{p}} R$ of all irreducible ideals with the patch topology (generated by all $S(a)$ and all $h(a)$ ) is a locally compact zero dimensional Hausdorff space. The analogue of the sheaf in 3.9 exists and is a sheaf of totally ordered $f$-rings over a locally Boolean space. This yields an embedding of any $f$-ring $R$ into the ring of global sections in a sheaf of totally ordered $f$-rings over a locally compact zero dimensional space, which is a projectable $f$-ring which is generated by the image of $R$ and the idempotents of the embedding ring. (Compare $3.9 \mathrm{ff}$.)

It should be recorded that a sectional representation theory for distributive lattices with 1 and 0 has been carried through in complete analogy to the GROTHENDIECK theory for commutative rings by BREZULEANU DiaconesCu [12], [13] and, independently by SelESNiCK [75]. With each lattice these authors construct a sheaf of local lattices (i.e. lattices in which the complement of $\{1\}$ is an ideal) over the space of prime ideals and show 
that the lattice is isomorphic to the lattice of all global sections in this sheaf. The relation between this theory and the character theory for lattices by KEIMEL and the author have not been systematically investigated.

\$5. The foundations of the general theory of sectional representation. In this section we attack the problem of finding a general theory of sectional representation which, on one hand, subsumes the applications which we saw in earlier sections, but is likewise suitable to represent topological rings and algebras by topological rings of continuous sections. Since the concept of a sheaf is ostensibly too narrow as we need nondiscrete stalks in whatever more general mathematical object we hope to operate. For some of the technical details we refer to DAUNS and Hofmann [20] where Dauns and I tried such a general theory for the first time. The emphasis has shifted from certain points to others, and this change is reflected in the following presentation. Some of our discussion may seem unnecessarily general; on the other hand it shows exactly why sectional representation techniques work in special cases. A slightly different approach based on topological rather than our uniform structure consideration has recently been offered by Mack [62a].

Uniform fields. We first pave the way for the concept of a field of uniform spaces which generalizes the idea of a sheaf of sets in the desired direction.

Definition 5.1. Let $\pi_{i}: E_{i} \rightarrow B$ be surjective functions, $i=1,2$. We denote with $\pi_{1} \vee \pi_{2}: E_{1} \vee E_{2} \rightarrow B$ the fibered product of $E_{1}$ and $E_{2}$; in other words $E_{1} \vee E_{2}$ is given by the pullback diagram

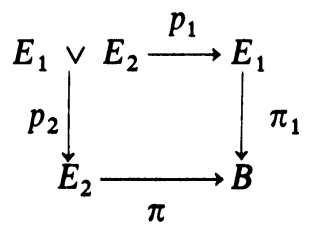

and $\pi_{1} \vee \pi_{2}=\pi_{i} p_{i}, i=1$, 2. (In terms of elements $E_{1} \vee E_{2}=\left\{\left(x_{1}, x_{2}\right)\right.$ $\left.\in E_{1} \times E_{2} \mid \pi_{1}\left(x_{1}\right)=\pi_{2}\left(x_{2}\right)\right\}$, and $p_{i}\left(x_{1}, x_{2}\right)=x_{i}$.) This concept, of course generalizes immediately to any family $\left\{\pi_{i} \mid i \in I\right\}$ of surjective functions onto $B$. If $\pi: E \rightarrow B$ is a surjective function, then a field uniform structure is a filter $\mathfrak{U}$ on $E \vee E$ such that the filter [ $\mathfrak{U}]$ generated by $\mathfrak{U}$ on $E \times E$ is a uniform structure of $E$.

An alternative description follows: If a family of uniform structures $\mathrm{ll}_{b}{ }_{b}$ of $\pi^{-1}(b), b \in B$, respectively, is given, then we obtain a unique structure $\mathfrak{l l}^{\prime}$ of the disjoint union $E$ of the $\pi^{-1}(b)$, such that the uniform space $\left(E, \mathfrak{U}^{\prime}\right)$ is the coproduct of the uniform spaces $\left(\pi^{-1}(b), \mathfrak{U}_{b}\right)$ in the category of uniform spaces. The filter $\mathfrak{U}$ of all $U \cap(E \vee E), U \in \mathfrak{U}^{\prime}$, is a field uniform structure, and every field uniform structure may be so described. We note $[\mathfrak{l}]=\mathfrak{l}^{\prime}$. 
Thus the difference between a field uniform structure and a coproduct uniform structure is purely technical and motivated by the field theoretic applications; indeed the concept of a field uniformity will allow us to speak later of "vertical closeness, i.e. closeness on the fibers $\pi^{-1}(b)$ ". In a sheaf, however, the entire topological structure of the sheaf space is "lateral" and we must imitate and generalize this idea until we arrive at the right synthesis generalizing both the concept of a bundle and the sheaf.

DEFINITION 5.2. Suppose that $\pi: E \rightarrow B$ is a continuous surjective map of topological spaces. If $V$ is an open subspace of $B$ then a local section over $V$ is a continuous map $\sigma: V \rightarrow E$ such that $\pi \sigma$ is the inclusion map $V \rightarrow B$. If $V=B$ then $\sigma$ is called a global section or just a section. The set of all sections is denoted by $\Gamma(\pi)$, the set of local sections over $V$ by $\Gamma(V, \pi)$. If $\sigma: V \rightarrow B$ is a (not necessarily continuous) function with $\pi \sigma=$ inclusion $V \rightarrow B$, then $\sigma$ is called a serration over $V$. The set of serrations over $V$ is denoted by $S(V, \pi)$. We write $S(\pi)=S(B, \pi)$.

The sections are the device to make precise what one means intuitively if one speaks of "shifting a point of $E$ continuously and laterally from one stalk into a neighboring stalk."

We will now single out those topologies on $E$ which are particularly suitable to accommodate the ideas of both "vertical closeness in stalks" and "continuity of lateral shifts."

Definition 5.3. Let $\pi: E \rightarrow B$ be a surjective continuous function of topological spaces and suppose that we have the following data:

(a) A field uniformity $\mathfrak{U}$ for $\pi$;

(b) a set $\Sigma$ of continuous local (and global) sections so that $E$ $=\bigcup\{\operatorname{im} \sigma \mid \sigma \in \Sigma\}$ (with the image im $\sigma$ of $\sigma$ ).

For a member $U \in \mathfrak{U}$ of the field uniform structure and a section $\sigma \in \Sigma$ we denote with $U(\sigma)$ the "tube" $\{x \in E \mid \pi(x) \in$ domain $\sigma$, and $(\sigma(\pi(x)), x) \in U\}$. We will call $(\pi, \mathfrak{U}), \pi: E \rightarrow B$ aniform field if the following condition is satisfied:

(c) The neighborhood filter of a point $x \in E$ has a basis of sets of the form $U(\sigma), U \in \mathfrak{U}, \sigma \in \Sigma, \sigma(\pi(x))=x$.

The sets $E_{b}=\pi^{-1}(b)$ are called the stalks of the field.

Trivial examples are given by constant fields $\pi: F \times B \rightarrow B$ with a uniform space $F$, and $\pi$ the projection. The field uniformity is the one induced on the stalks $F \times\{b\}$ by the uniformity of $F$.

It is useful to observe that condition (c) has an equivalent version in which an arbitrarily selected and conceivably much smaller family of sections replaces the full set of local sections:

Proposition 5.4. A continuous surjective function $\pi: E \rightarrow B$ together with a field uniformity $\mathfrak{l l}$ is a uniform field if and only if the following condition is satisfied: 
(c') There is a function $x \mapsto \sigma_{x}$ from $E$ into the set $\Gamma^{\prime}$ of all continuous local sections such that (i) $\sigma_{x}(x)=x$ and that (ii) the neighborhood filter of $x$ in $E$ has a basis of sets of the form $U\left(\sigma_{x} \mid V\right)$, where $U$ ranges through a hasis of $\mathfrak{U}$ and $V$ through an open neighborhood basis of $\pi(x)$ in $B$.

(For a proof see $[20$, p. 4].)

Note that in general the sets $U(\sigma)$ need not be open.

The following is the immediate:

Proposition 5.5. If ( $\pi, \mathfrak{U}), \pi: E \rightarrow B$ is a uniform field, then $\pi$ is an open continuous map and therefore a quotient map. For any subset $B^{\prime} \subseteq B$ the field uniformity induces a field uniformity $\mathfrak{U}^{\prime}$ for $\pi^{\prime}: E^{\prime} \rightarrow B^{\prime}$ where $E^{\prime}=\pi^{-1} B^{\prime}$ and $\pi^{\prime}=\pi \mid E^{\prime}$, and with the restriction of local sections, too, one obtains a uniform field $\left(\pi^{\prime}, \mathfrak{U}^{\prime}\right), \pi^{\prime}: E^{\prime} \rightarrow B^{\prime}$. On each stalk $\pi^{-1}(b)$ the induced topology and the topology defined by the uniform structure of $E_{b}$ originating from the field uniformity agree.

LEMMA 5.6. On the set $S(\pi)$ of all serrations we obtain a natural uniform structure as follows: For each element $U$ of the field uniform structure $\mathfrak{U}$ we define the set $\underline{U} \subset S(\pi) \times S(\pi)$ to be the set of all pairs $(\sigma, \tau)$ with $(\sigma(b)$, $\tau(b)) \in U$ for all $b \in B$. Then the set $\{\underline{U} \mid U \in \mathfrak{U}\}$ is a basis for a uniform structure of $S(\pi)$.

It will be understood from now on that $S(\pi)$ (and accordingly all $S(V, \pi)$ for any open set $V$ of $B$ ) have this uniform structure and that $\Gamma(\pi)$ and the $\Gamma(V, \pi)$ have the induced uniform structure. Since completeness of uniform spaces is the most basic of all pertinent general concepts we observe

Proposition 5.7. Let $(\pi, \mathfrak{U}), \pi: E \rightarrow B$ be a uniform field. If all stalks are complete, then $S(\pi)$ and $\Gamma(\pi)$ are complete; in particular, $\Gamma(\pi)$ is closed in $S(\pi)$.

(Proof see [20, p. 7].)

We anticipate that (just as in the theory of function spaces) the idea of boundedness will play an important role. Yet in the absence of base points (such as we have in case of groups) the concept of boundedness in general is a little more delicate.

Let $(\pi, \mathfrak{U})$ be a uniform field. If $U \in \mathfrak{U}$ and $n$ is a natural number we denote by $U^{n}$ the relation inductively defined by $U^{n}=U^{n-1} U, n>1$, where juxtaposition of relations signifies the relation product. We then define an equivalence relation on $S(\pi)$ (resp. on $\Gamma(\pi)$ ) by $\beta_{U}=\{(\sigma, \tau)$ |there is a natural number $n$ such that $(\sigma(b), \tau(b)) \in U^{n}$ for all $\left.b \in B\right\}$. (In the uniform space $S(\pi)$ there is a familiar equivalence relation $\alpha_{U}$, relative to which two elements $\sigma$ and $\tau$ are related whenever there is a finite sequence $\sigma_{0}=\sigma, \ldots, \sigma_{n}=\tau$ with $\left(\sigma_{i}, \sigma_{i-1}\right) \in \underline{U}$ for $i=1, \ldots, n$, in other words if $(\sigma, \tau) \in \underline{U}^{n}$ for some $n$.) Obviously, $\alpha_{U} \subseteq \beta_{U}$, but the converse does not hold (see $\left[20\right.$, p. 9, 1.23]). We define $\beta=\bigcap\left\{\beta_{U}: U \in \mathfrak{U}\right\}$. 
DEFINITION 5.8. Let $(\pi, \mathfrak{U})$ be a uniform field. We say that $S(\pi)$ and $\Gamma(\pi)$, respectively, are semiconnected if and only if $S(\pi)$ and $\Gamma(\pi)$ have only one $\beta$-component, respectively. The relation $\beta$ is called the semiconnectivity relation.

We then have the following proposition:

Proposition 5.9. Suppose that $(\pi, \mathfrak{U})$ is a uniform field. Then $\Gamma(\pi)$ is semiconnected if $B$ is quasi-compact and all stalks $E_{b}$ are connected.

For the applications of the theory to topological algebra the connectivity of the stalks is a mild hypothesis since all real topological vector spaces are connected. However, the assumption that the basis be quasi-compact is unfortunately not satisfied often enough. We therefore are interested in the possibility of singling out sections which should be called bounded.

It is exactly for this purpose that this semiconnectivity is introduced. We will later have a distinguished section in a uniform field, namely the zero section in a field of topological groups. The bounded sections then are exactly the sections in the semicomponent of the zero section. That this idea agrees with the more traditional idea of boundedness is most easily recognized if the field uniformity for $\pi$ is given by a function $d: E \vee E \rightarrow R^{+}$ into the nonnegative reals which induces a metric on each stalk and is such that the field uniformity has a basis of sets $U_{r}=\{(x, y) \in E \times E \mid d(x, y)<r\}$. Then two sections are in the same semicomponent if for some real number $C$ we have $\sup \{d(\sigma(b), \sigma(b)) \mid b \in B\} \leqq C$. In this sense, Proposition 5.9 generalizes the traditional fact that continuous real valued functions on a compact space are bounded.

In conventional function space theory, separation properties of the domain space can often be expressed in terms of sections. Such conclusions remain relevant in the context of uniform fields. The following definitions are convenient :

Definition 5.10. Let $(\pi, \mathfrak{U})$ be a uniform field. We say that the field uniformity $\mathfrak{U}$ and a set $\Sigma$ of global sections are separating (resp. strongly separating), if for two different base points $b, c \in B$ there are $U \in \mathfrak{U}$ and sections $\sigma, \tau \in \Sigma$ with $\sigma(b)=\tau(b)$ such that $\sigma(c) \notin U(\tau)$ (resp. $\sigma(c) \notin U(\tau)^{-}$, the closure of $U(\sigma)$ in $E$ ). We say that $\mathfrak{U}$ is smooth for $\Sigma$ if for each $\sigma \in \Sigma$ and $U \in \mathfrak{U}$ there is a $V \in \mathfrak{U}$ with $V(\sigma)^{-} \cap \pi^{-1}($ domain $\sigma) \subseteq U(\sigma)$. We then have

Proposition 5.11. If in a uniform field, $\mathfrak{U}$ and $\Gamma(\pi)$ are strongly separating, then the base space is Hausdorff. If, in addition, $\mathfrak{U}$ is smooth, then the base space $B$ is regular Hausdorff.

After we have inspected some of the properties of uniform fields and their global sections, we turn to the question of the existence of uniform fields. While, it is true, there are quite general existence theorems, the right 
degree of generality, which so far has sufficed for all applications I know, is given by the setting of transformation groups, from which uniform fields arise naturally. This we are discussing in the following:

DefinItion 5.12. Let $G$ be a group acting on the set $E$ in detail: There is a function $(g, x) \mapsto g x: G \times E \rightarrow E$ with $1 x=x$ and $(g h) x=g(h x)$ for all $x \in E$ and all $g, h \in G$ with identity 1 of $(G)$. Let $B=E / G$ be the orbit space and $\pi: E \rightarrow B$ the orbit projection.

Suppose that $\mathfrak{U}$ is a field uniformity making $(\pi, \mathfrak{U})$ into a uniform field. We say that $(\pi, \mathfrak{l})$ is a homogeneous uniform field if all functions $x \mapsto g x$ : $E \rightarrow E, g \in G$ are continuous and for each $U \in \mathfrak{U}$ and each $g \in G$ there is a $V \in \mathfrak{U}$ such that $(g x, g y) \in U$ for all $(x, y) \in V$. We say that $(\pi, \mathfrak{U})$ is a uniformly homogeneous uniform field if in addition, $G$ is a topological group and for each $U \in \mathfrak{U}$ and each $g \in G$ there is a $V \in \mathfrak{U}$ and a neighborhood $N$ of the identity of $G$ such that $(g x, h y) \in U$ for all $(x, y) \in V$ and all $(g, h) \in G \times G$ with $h g^{-1} \in N$. The group $G$ is called the structure group.

It is clear that for a homogeneous uniform field the group $G$ operates on the set of serrations $S(V, \pi)$ under $(g \sigma)(b)=g \sigma(b)$, and that $\Gamma(V, \pi)$ is invariant under $G$ for all open $V \subseteq B$. One shows the following without difficulty [20, p. 16].

Proposition 5.13. If $(\pi, \mathfrak{U}), \pi: E \rightarrow B$ is a uniformly homogeneous uniform field with structure group $G$, then $G$ is a topological transformation group of $E$, the topology of $B$ is the orbit space topology, and $G$ acts as a topological transformation group on $\Gamma(V, \pi)$ for all open $V \subseteq B$.

The noteworthy fact which figures prominently in applications is that one has a general existence theorem for uniformly homogeneous uniform fields. Indeed, suppose that $G$ is a topological group and $E$ a set on which $G$ acts on the left. We let $\pi: E \rightarrow B$ be the orbit projection and assume that we are given a set $\Sigma$ of right inverses $\sigma: B \rightarrow E$ of $\pi$ such that $E=\{\sigma(B) \mid \sigma \in \Sigma\}$. The set $\Sigma$ will be called a full set of serrations. We then define a filter on $E \vee E$ by the basic sets $U^{\prime}=\{(x, y) \in E \vee E \mid y \in U x\}$ where $U$ ranges through the filter basis $\mathfrak{B}$ of open identity neighborhoods of $G$. This filter is called the field uniformity determined by the action of $G$. The collection $\left\{U^{\prime}(\sigma) \mid U \in \mathfrak{B}, \sigma \in \Sigma\right\}$ is a subbasis for a topology, called the field topology determined by the operation of $G$. We then have the following principal result :

THEOREM 5.14. If a topological group $G$ operates on the left on a set $E$ and we have a full set $\Sigma$ of serrations, which is invariant under the action of $G$, then we have the following conclusions:

(1) $G$ is a topological transformation group on $E$ when $E$ is given the field topology determined by the operation of $G$. 
(2) The orbit space topology (quotient topology) on $B$ and the finest topology of $B$ making all serrations $\sigma$ of $\Sigma$ continuous agree.

(3) $(\pi, \mathfrak{U}), \pi: E \rightarrow B$ is a uniformly homogeneous uniform field.

(4) For each $x \in E$ let $G_{x}$ be the isotropy group of $G$ at $x$. Then the function $\psi_{x}: G / G_{x} \rightarrow E_{\pi(x)}$ defined by $\psi_{x}\left(g G_{x}\right)=g x$ is an isomorphism of uniform spaces if $G / G_{x}$ has the right quotient uniform structure (given by the basic entourages $\left\{\left(g G_{x}, h G_{x}\right) \mid h \in U g\right\}$ where $U$ ranges through a basis of open identity neighborhoods).

(5) If $G$ has a basis of identity neighborhoods which are invariant under all inner automorphisms (i.e. if the right and left uniform structure on $G$ agree), then the operation $(\mathrm{g}, x) \mapsto g x: G \times E \rightarrow E$ is uniformly continuous relative to the right uniform structure of $G$ and the uniform structure $[\mathfrak{U}]$ of $E$ (i.e. if $U \in \mathfrak{U}$, then there is an identity neighborhood $N$ of $G$ and $a V \in \mathfrak{U}$ such that $(g x, h y) \in U$ whenever $h g^{-1} \in N$ and $\left.(x, y) \in V\right)$. In other words, $(\pi, \mathfrak{u})$ is uniformly homogeneous.

(6) If $\varepsilon$ and $\omega$ are topologies on $E$ and $B$ such that $(\pi, \mathfrak{U}), \pi:(E, \varepsilon) \rightarrow(B, \omega)$ is a uniform field relative to which $\Sigma$ is a set of sections, then $\varepsilon$ is at least as fine as the topology determined by the action of $G$, and $\omega$ is at least as fine as the topology given by (2) above.

The topology produced on the orbit space $B$ by $(2)$ has been called the weak *-topology, but for reasons of conflict with established terminology we call it the field *-topology or field star topology. A group $G$ is called an SIN-group (for small invariant neighborhoods) if it satisfies the hypothesis of 5.14.5.

The canonical field. Recall that by 5.11 the group $G$ operates as a topological transformation group on the uniform space $\Gamma(\pi)$ leaving the subspace $\Sigma$ invariant. In most applications of Theorem 5.14 the data are given in the following form: We have a topological group $G$ and family of subgroups $K_{b}, b \in B$; we define $G / K_{b}$ to be the space of right quotients $g K_{b}$ and set $E=\left\{G / K_{b} \times\{b\} \mid b \in B\right\}$ and let $G$ operate on $E$ by $g\left(h K_{b}, b\right)$ $=\left(g h K_{b}, b\right)$. The orbit map may then be replaced by the function $\pi: E$ $\rightarrow B$ given by $\pi\left(g K_{b}, b\right)=b$. We obtain a full set $\hat{G}$ of serrations $\hat{g}: B \rightarrow E$ by defining $\hat{g}(b)=\left(g K_{b}, b\right)$, and $\hat{G}$ is obviously invariant under the action of $G$. Thus Theorem I applies and provides a uniformly homogeneous uniform field $(\pi, \mathfrak{U})$ which is unique in the sense of 1.14 .6 , and for which $G$ is a space of continuous sections. The function $g \mapsto \hat{g}: G \rightarrow \Gamma(\pi)$ is uniformly continuous, and is injective if and only if $\bigcap\left\{K_{b} \mid b \in B\right\}=\{1\}$. Even in this case the corestriction $G \rightarrow \hat{G}$ of this function need not be open.

DEFINITION 5.15. If a uniformly homogeneous field $(\pi, \mathfrak{U})$ arises from a topological group $G$ and a family $\left\{K_{b} \mid b \in B\right\}$ of subgroups as described above, then $(\pi, \mathfrak{u})$ is called the canonical field associated with $G$ and $\left\{K_{b} \mid b \in B\right\}$. 
In the canonical field whose topology is defined by a particularly economic initial set of serrations $G$ (which is just one orbit of the set of all serrations under the action of $G$ ) we have some additional properties which are worth noticing:

Proposition 5.16. Let $(\pi, \mathfrak{U})$ be the canonical field associated with $G$ and $\left\{K_{b} \mid b \in B\right\}$. Then for any open identity neighborhood $U$ of $G$ the tube $U^{\prime}(g)$ is open for any $g \in G$.

Each of the following conditions suffice for the field *-topology on the base space to be Hausdorf:

(a) $G \backslash \bigcup\left\{K_{b} \mid b \in B\right\}=\bigcap\left\{G \backslash K_{b} \mid b \in B\right\}$ has a nonempty interior $W$ and for different base points $b, c \in B$ we have $K_{b} K_{c} \cap W \neq \varnothing$ or $K_{c} K_{b} \cap W \neq \varnothing$.

(b) $b \neq c$ in $B$ implies $K_{b} \neq K_{c}$ and $\bigcap\left\{U^{\prime}(1)^{-} \mid U^{\prime} \in \mathfrak{U}\right\}=1(B)$.

(See [20, p. 17, 1.51 or p. 28, 2.26].)

Note that $W=G \backslash\left(\bigcup\left\{K_{b} \mid b \in B\right\}\right)^{-}$and that sometimes it is not too hard to verify this condition; e.g. if $G$ is a normed algebra with identity then the group of units is open and fails to meet any ideal, so that this condition is satisfied if all $K_{b}$ are ideals. The condition $\left(K_{b}+K_{c}\right) \cap W \neq \varnothing$ would in this case certainly be satisfied if $K_{b}$ and $K_{c}$ are two maximal ideals. In the discrete case, of course $W$ is just the complement of the union of the $K_{b}$.

A frequent case in the applications is the case that the $K_{b}$ are actually normal subgroups, in which case all stalks are topological groups in their own right. This should give the field additional structure. We therefore introduce the concept of a uniform field of topological groups.

Definition 5.17. Let $(\pi, \mathfrak{l}), \pi: E \rightarrow B$ be a uniform field such that the following additional conditions are satisfied:

(a) All stalks $E_{b}$ are groups and the function $(x, y) \mapsto x^{-1} y: E \times E \rightarrow E$ is continuous.

(b) The field uniformity induces on each stalk the left (resp. right) uniform structure given by the topological group structure of the stalk induced by (a).

(c) For any $U \in \mathfrak{U}$ there is a $W \in \mathfrak{U}$ such that $(x, s),(y, t) \in W$ and $\pi(x)=\pi(y)$ imply $\left(x^{-1} y, s^{-1} t\right) \in U$ (resp., $\left.\left(y x^{-1}, t s^{-1}\right) \in U\right)$.

Then $(\pi, U)$ is called a left (resp. right) uniform field of topological groups.

If all stalks are abelian groups, the distinction between the left and the right uniform structures become immaterial.

Proposition 5.18. If $(\pi, \mathfrak{U})$ is a uniform field of topological groups, then for each open $V \subseteq B$ the uniform space $\Gamma(V, \pi)$ is in fact a topological group relative to the operations given by $\left(\sigma^{-1} \tau\right)(v)=\sigma(v)^{-1} \tau(v)$ for $v \in V$; and our standard uniform structure on $\Gamma(V, \pi)$ is the left (resp. right) uniform structure derived from this topological group structure.

If we now recall the concept of semiconnectivity of $S(\pi)$ or $\Gamma(\pi)$ discussed 
in 5.8 we note that it may be shown that the relation of semiconnectivity is a congruence on $\Gamma(\pi)$. Let $\Gamma^{b}(\pi)$ denote the semicomponent of the identity section 1. Then the sections in $\Gamma^{b}(\pi)$ are called bounded.

Proposition 5.19. The set $\Gamma^{b}(\pi)$ of all bounded sections in a uniform field of topological groups is a closed normal subgroup of $\Gamma(\pi)$.

By 5.7 we know that every section is bounded if the base space is quasicompact and all stalks are connected.

It is, however, not only the subgroup of bounded sections which plays a role in the theory, but also the subgroup of sections which vanish at infinity in a suitable sense. Indeed let $\mathfrak{S}$ be a filter in $B$. Let $\Gamma_{\mathfrak{G}}(\pi)$ be the set of all sections $\sigma$ such that the sets $\sigma^{-1}\left(U^{\prime}(1)\right)$ where $U$ runs through the filter of neighborhoods of 1 in $G$ generate a filter which contains $\mathfrak{H}$. In a loose way we might say that $\sigma \in \Gamma_{\mathfrak{H}}(\pi)$ iff $\lim \sigma=0$. Then $\Gamma_{\mathfrak{H}}(\pi)$ is a closed subgroup of $\Gamma(\pi)$. Frequently $\mathfrak{H}$ will be generated by complements of an ideal of quasi-compact sets of $B$.

With the aid of the canonical uniform field associated with a topological group and a family $\left\{K_{b} \mid b \in B\right\}$ of normal subgroups we obtain the following corollary to Theorem 5.14 :

THEOREM 5.20 (The canonical existence and uniqueness theorem. DAUNS and HoFMANN [20]). Let $G$ be a topological SIN-group and $\left\{K_{b} \mid b \in B\right\} a$ family of normal subgroups. Let $E=\bigcup\left\{G / K_{b} \times\{b\} \mid b \in B\right\}$ and $\pi: E \rightarrow B$ the function $\pi\left(g K_{b}, b\right)=b$. Then there is a field uniformity $\mathfrak{U}$ and there are topologies on $E$ and $B$ such that

(1) $(\pi, \mathfrak{U}), \pi: E \rightarrow B$ is a uniform field of topological groups and the topology of $E$ has the subbasic open sets $U^{\prime}(\hat{g})$, where $U$ ranges through the open neighborhoods of 1 in $G$ and $g \in G$.

(2) The topology of $B$ is at the same time the finest one making $\pi$ continuous and the coarsest one making all $\hat{g}: B \rightarrow E$ continuous. It is given by the subbasic sets $\left\{b \mid g \in U K_{b}\right\}, U$ and $g$ as in (1).

(3) The function $g \mapsto \hat{g}: G \rightarrow \Gamma(\pi)$ is a continuous topological group morphism with kernel $\bigcap\left\{K_{b} \mid b \in B\right\}$. It is an isomorphism of topological groups onto the image iff for any identity neighborhood $U$ of $G$ there is a neighborhood $V$ of 1 in $G$ such that $\bigcap\left\{V K_{b} \mid b \in B\right\} \subseteq U$. If $G$ is connected, then $\hat{G} \subseteq \Gamma^{b}(\pi)$.

(4) The function $g \mapsto\left(g K_{b}, b\right): G \rightarrow E_{b}$, is a quotient morphism of topological groups for each $b \in B$.

(5) The topologies of $E$ and $B$ are minimal relative to the property of making $\pi: E \rightarrow B$ into a uniform field relative to the field uniformity $\mathfrak{U}$ and relative to the requirement that all sections $\hat{\mathrm{g}}, g \in G$ be continuous.

We continue to call the morphism of (3) the Gelfand representation (or Gelfand morphism). 
For the benefit of the algebraist we give a purely algebraic version of this existence and uniqueness theorem:

Corollary 5.21 (Dauns and HofmanN [20]). If $G$ is a group and $\left\{K_{b} \mid b \in B\right\}$ a family of subgroups, then there is a unique structure of a sheaf of groups on $E=\bigcup\left\{G / K_{b} \times\{b\} \mid b \in B\right\}$ relative to the function $\pi: E \rightarrow B$ as sheaf projections so that the Gelfand representation $g \mapsto \hat{g}: G \rightarrow \Gamma(\pi)$ into the group of all continuous sections is well defined, and which is the coarsest with these properties. The topology in $E$ is generated by the finite intersections of the $\hat{g}(B), g \in G$ as basis and the field *-topology on $B$ is generated by the projections of these sets.

For rings, this theorem was also established by Mulvey [67]. Note that the field *-topology on $B$ is generated by the sets $h(a), a \in G$ where $h(a)$ $=\left\{b \in B \mid a \in K_{b}\right\}$ whereas, in the ring case, the hull-kernel topology is in fact generated by the complements $S(a)=B \backslash h(a)$. We should refer back to 3.9, where the patch topology appears as a refinement of the field *-topology and the hull-kernel topology.

Mulvey's adjunction. If $R$ is a ring with identity and $\left\{I_{b} \mid b \in B\right\}$ a fixed family of ideals such that the quotient morphisms $R \rightarrow R / I_{b}$ separate, then one obtains from Corollary 5.21 a sheaf $\pi: E \rightarrow B$ of rings with identity with the stalks $E_{b}=R / I_{b} \times\{b\} \cong R / I_{b}$, and the Gelfand morphism $R \rightarrow \Gamma(\pi)$ is injective. This reduces the study of a ring $R$ with a given subdirect representation and of the modules over such a ring essentially to the study of ringed spaces $\pi: E \rightarrow B$ together with a subring $R$ of $\Gamma(\pi)$ such that $\{r(b) \mid r \in R\}=E_{b}$ for all $b \in B$. This is the approach which was worked out in great detail by MULVEY [67]. Some brief indications of the essence of this approach are in place, in particular since the problem of carrying a similar program through in the case of fields has only had some tentative beginnings such as the work of TAKAHASHI [79].

We write $\mathscr{R}$ for the sheaf of rings over the base space $B$ and denote the distinguished subring of $\mathscr{R}(B)$ by $R$. Let $M$ be an $R$-module. We apply the technique of 1.4 (which we will generalize in $\S 6$ ) to $M$ and the family of abelian subgroups $\left\{I_{b} \cdot M \mid b \in B\right\}$. In other words we define $\tilde{K}_{b}=$ $\bigcup_{b \in U} \bigcap\left\{I_{b} \cdot M \mid b \in U\right\}$ where $U$ ranges through the neighborhoods of $b$ in $B$. The basic neighborhoods of $b$ are given by $h(F)=\left\{c \in B \mid F \subseteq I_{c}\right\}$ where $F$ is a finite subset of $I_{b}$. Thus $\tilde{R}_{b}=\bigcup_{F \subseteq I_{b}} \bigcap\left\{I_{c} \cdot M \mid F \subseteq I_{c}\right\}$.

We know from 1.4 that there is a sheaf of abelian groups over $B$ with stalks $\mathscr{M}_{b} \cong M / K_{b}$. Just as in the discussion following 1.19 we obtain a bilinear sheaf map $\mathscr{R} \times \mathscr{M} \rightarrow \mathscr{M}$, which makes $\mathscr{M}$ into an $\mathscr{R}$-module. However, due to the choice of a very specific topology on $B$, namely the field star topology induced by the family of the $I_{b}$, we are in a better position to identify the stalks $\mathscr{M}_{b}$ as we were then (in §1) where an otherwise unidentified topology was given on $B$. 
Lemma 5.22. We have $\tilde{K}_{b}=I_{b} \cdot M$ and thus $\mathscr{M}_{b}=M /\left(I_{b} \cdot M\right)$ for all $b \in B$.

Proof. Trivially, $\widetilde{K}_{b} \subseteq I_{b} \cdot M$. We have to show the converse. Let $m \in I_{b} \cdot M$; then there are elements $r_{k} \in I_{b}, m_{k} \in M, k=1, \ldots, n$, such that $m=\sum r_{k} \cdot m_{k}$. Now $U=h\left\{r_{1}, \ldots, r_{n}\right\}$ is a neighborhood of $b$ in $B$ and $c \in U$ means exactly $r_{k} \in I_{c}$ for $k=1, \ldots, n$. This implies that $m=\sum r_{k} \cdot m_{k} \in I_{c} \cdot M$, hence $m \in \bigcap\left\{I_{c} \cdot M \mid c \in U\right\}$, and therefore $m \in \tilde{K}_{b}$.

The assignment $M \mapsto \mathscr{M}$ is functorial on the category $R$-Mod of $R$ modules and thus extends to a functor $L$ into the category $\mathscr{R}$-Mod of $\mathscr{R}$-modules. The assignment which associates with any $\mathscr{R}$-module $\mathscr{M}$ the $R$-module $\mathscr{M}(B)$ of its global sections, is likewise functorial, where the $R$-module operation on $\mathscr{M}(B)$ is given by $(r \cdot \sigma)(b)=\left(r+I_{b}\right) \cdot \sigma(b)$ with the $R / I_{b}=\mathscr{R}_{b}$-module operation in $\mathscr{M}_{b}$. Let us denote the functor $\mathscr{R}$-Mod $\rightarrow R$-Mod which we have thus defined by $\Gamma$. If $\mathscr{M}$ is an arbitrary $\mathscr{R}$-module, then we can define a morphism $\varepsilon_{\mathscr{M}}: L \Gamma \mathscr{M} \rightarrow \mathscr{M}$ of $\mathscr{R}$-modules by $\varepsilon_{\mathscr{M}}\left(\sigma+I_{b} \cdot \Gamma \mathscr{M}, b\right)=\sigma(b)$, since all sections in $I_{b} \cdot \Gamma \mathscr{M}$ vanish because $\mathscr{M}_{b}$ is an $R / I_{b}$-module. The claims that $\varepsilon_{\mu}$ is a sheaf and in fact an $\mathscr{R}$-module morphism are readily checked. Let $\mathscr{M} \in \mathscr{R}-M o d$ and $N \in R$-Mod and let $f: L N \rightarrow \mathscr{M}$ by an $\mathscr{R}$-Mod-map. Then we produce an $R$-Mod map $f^{\prime}: N$ $\rightarrow \Gamma \mathscr{M}$ by defining $f^{\prime}(n)(b)=f\left(n+I_{b} \cdot N, b\right)$, where $n \in N, b \in B$. The element $\left(L f^{\prime}\right)\left(n+I_{b} \cdot N, b\right)$ in $L \Gamma \mathscr{M}$ is given by $f^{\prime}(n)+I_{b} \cdot \Gamma \mathscr{M}$ by the way the functor $L$ is defined on morphisms; if we apply $\varepsilon_{\mu}$ we obtain $\varepsilon_{\text {II }}\left(f^{\prime}(n)+I_{b} \cdot \Gamma \mathscr{M}, b\right)=f^{\prime}(n)(b)$ which was exactly $f\left(n+I_{b} \cdot M, b\right)$. Thus $\varepsilon_{M}\left(L f^{\prime}\right)=f$, and $f^{\prime}$ is certainly uniquely determined by this relation. Thus we have obtained a bijection $f \mapsto f^{\prime}: \mathscr{R}-\operatorname{Mod}(L N, \mathscr{M}) \rightarrow R-\operatorname{Mod}(N, \Gamma \mathscr{M})$ which precisely guarantees that $L$ is left adjoint to $\Gamma$ in such a way that $\varepsilon_{\mathscr{M}}$ is the co-unit (= back adjunction).

The unit (= front adjunction) $\gamma_{N}: N \rightarrow \Gamma L N$ is given by $\left(1_{L N}\right)^{\prime}$, where $1_{L N}: L N \rightarrow L N$ is the identity, and it turns out that it is exactly the Gelfand morphism of $N$. Its kernel is $\bigcap\left\{I_{b} \cdot N \mid b \in B\right\}$. We have thus established the following result:

Proposition 5.23 (MulveY's adjunction theorem [67], [68]). Let $R$ be a ring with identity and $\left\{I_{b} \mid b \in B\right\}$ a fixed family of ideals. Let $\mathscr{R}$ be the canonical sheaf of rings with identity over the space $B$ which is endowed with the field star topology. Then the global section functor $\Gamma$ for the category $\mathscr{R}$-Mod of $\mathscr{R}$-modules over $B$ into the category $R$-Mod of $R$-modules has a left adjoint $L$ which associated with each $R$-module $M$ an $\mathscr{R}$-module over $B$ with stalks $(L M)_{b} \cong M /\left(I_{b} \cdot M\right)$. The front adjunction $\gamma_{M}: M \rightarrow \Gamma L M$ is the Gelfand morphism with kernel $\bigcap\left\{I_{b} \cdot N \mid b \in B\right\}$. The back adjunction $\varepsilon_{\mathscr{M}}: \mathscr{M}$ $\rightarrow L \Gamma \mathscr{M}$ is given by $\varepsilon_{\mathscr{M}}\left(\sigma+I_{b} \cdot \Gamma \mathscr{M}, b\right)=\sigma(b)$.

If we wish to apply MULVEY's adjunction theorem to the developments of $\S 1$, we arrive at the following conclusion: Let $B \subseteq \operatorname{Spec} R$ be a space of 
prime ideals of the ring $R$ with identity such that $\bigcap B=\{0\}$. Let $I_{b}$ $=\bigcup_{a \notin b} a_{B}^{\perp}$; then the field star topology on $B$ which is used in the construction of $L R$, is equal to or coarser than the hull-kernel topology by 5.21 . In the establishment of the MULVEY adjunction it was necessary that we used the field star topology in order to obtain $(L M)_{b} \cong M /\left(I_{b} \cdot M\right)$. In general, the hull-kernel topology on $B$ and the field star topology induced from $\{\tilde{I} \mid I \in B\}$ do not agree. Indeed for the two topologies to agree, it is necessary and sufficient, that for each $I \in B$, and each hull-kernel neighborhood $U$ of $I$ there is a field star neighborhood $V$ of $I$ contained in $U$; equivalently for all $I \in B$ and all $a \in R \backslash I$ there is a finitely generated ideal $F$ contained in $\tilde{I}$ such that $F \subseteq \tilde{J}, J \in B$ implies $a \notin J$. Let us observe that $F \subseteq \tilde{I}$, if $F$ is finitely generated, means that there is an $a_{1} \notin I$ with $F \subseteq a_{B}^{\perp}$ $=a^{\perp}$ (since $\left.\bigcap B=\{0\}\right)$. Thus we have

LEMMA 5.24. If $B$ is a set of prime ideals of a ring $R$ with $\bigcap B=\{0\}$, then the hull-kernel topology of $B$ agrees with the field star topology associated with the family $\{\tilde{I} \mid I \in B\}$ if and only if for all $I \in B$ and all $a \notin I$ there is an element $a_{1} \notin I$ and a finitely generated ideal $F$ with $F \subseteq a_{1}^{\perp}$ such that for any $J \in B$ the existence of an $a_{2} \notin J$ with $F \subseteq a_{2}^{\frac{1}{2}}$ implies $a \notin J$.

While it is not so easy to see through this characterization of the equality of hull-kernel and field star topology, certain sufficient conditions are easier to understand. If, e.g., the hull-kernel topology is compact Hausdorff and the field star topology is Hausdorff (see 5.16) then the two topologies must be equal since compact topologies are minimal among Hausdorff ones. Indeed we have the following

Proposition 5.25. Let $R$ be a ring with identity and $B=\operatorname{Max} R$. Then the hull-kernel topology and the field star topology induced by $\{\tilde{I} \mid I \in \operatorname{Max} R\}$ agree on $B$.

Proof. Let $I \in S(a)=S_{B}(a)$ in $B$; then $h(a)=h_{B}(a)=B \backslash S(a)$ is closed and hence quasi-compact in $B$, since $B$ itself is quasi-compact. For every $J \neq I$ the set $\{I, J\}$ is closed in $B$, since $B=\operatorname{Max} R$ is $T_{1}$. Thus by 1.16 , there is an $a_{J} \in R$ with $a_{J} \in \tilde{I}$ and $a_{J}-1 \in \tilde{J} \subseteq J$; in particular $a_{J} \notin J$. By the quasi-compactness of $h(a)$ we find elements $a_{1}, \ldots, a_{n} \in \tilde{I}$ such that $h(a) \subseteq S\left(a_{1}\right) \cup \cdots \cup S\left(a_{n}\right)$. If we let $F$ be the ideal generated by $a_{1}, \ldots, a_{n}$, then $F \subseteq \tilde{I}$ and thus $I \in\{J \in B \mid F \subseteq \widetilde{J}\} \subseteq h(F)=h\left(\left\{a_{1}, \ldots, a_{n}\right\}\right)=h\left(a_{1}\right)$ $\cap \cdots \cap h\left(a_{n}\right) \subseteq S(a)$. Since $\{J \in B \mid F \subseteq \tilde{J}\}$ is a basic neighborhood of $I$ relative to the field star topology induced by $\{\mathcal{J} \mid J \in B\}$, this topology is finer than or equal to the hull-kernel topology. The converse is always true. Hence the assertion.

This proposition shows that MulveY's theory applies, e.g., to any strongly semisimple ring $R$ with identity and the ideal set $\{\tilde{I} \mid I \in \operatorname{Max} R\}$. A 
proof which is completely analogous to the one in Proposition 5.25, but in which the application of 1.16 is replaced by an application of 1.28 yields

Proposition 5.26. Let $R$ be a strongly harmonic ring with identity and $B=\operatorname{Max} R$. Then the hull-kernel topology and the field star topology induced by $\{I \mid I=\operatorname{Max} R\}$ agree on $B$.

Thus, as another example, MuLveY's setup works for all strongly harmonic rings $R$ and the ideal set $\{\check{I} \mid I \in \operatorname{Max} R\}$. This includes all weakly biregular, hence all biregular and thus, in particular, commutative regular rings. In this fashion one recovers the adjunction described by Pierce [72].

The property which was essentially used in the proof of 5.25 and 5.26 was in the first case a property of the ring $R=\mathscr{R}(B)$ of global sections in the sheaf $\mathscr{R}$ and in the second case a property of the ring $R=\mathscr{R}^{\prime}(B)$ of global sections in $\mathscr{R}^{\prime}$, namely that for a hull-kernel open neighborhood $U$ of a base point $b$ there was a section $a$ in $R$ whose support is contained in $U$ and which takes the value 1 in $b$ (see 1.16 ii and 1.28). If, more generally, $R$ is a subring of $\mathscr{R}(B)$ for a ringed space $\mathscr{R}$, then MULveY calls $R$ completely regular in $\mathscr{R}$ if it has this property and if, in addition, the base space $B$ is Hausdorff. The ring $R$ is called compact in $\mathscr{R}$ if it is completely regular in $\mathscr{R}$ and the base space is compact. In the latter case one has necessarily $R=\mathscr{R}(B)$ [67]. Compactness of $R$ in $\mathscr{R}$ for a completely regular $R$ in $\mathscr{R}$ is equivalent to saying that every maximal ideal of $R$ contains some $I_{b}$ $=\{a \in R \mid a(b)=0\}[67,7.1]$ and in addition these conditions are equivalent to the condition that the adjunction $(L, \Gamma)$ of 5.23 is an equivalence $[67,8.1]$. This, then, applies not only to all strongly semisimple harmonic rings $R$ with identity and the family $\{\tilde{I} \mid I \in \operatorname{Max} R\}$, but also, in view of what has been said above, to all strongly harmonic rings $R$ with identity and the family $\{\check{I} \mid I \in \operatorname{Max} R\}$. If MULVEY's arguments should not depend on the Hausdorffness of the base space, we would in fact have that $(L, \Gamma)$ is an equivalence for all strongly semisimple rings with identity whether they are harmonic or not.

§6. Given topologies on the base space. In $\S 5$ we have seen that the problem of sectional representation has a general and in some sense canonical solution. One of the most unsatisfactory features is that in the most interesting cases (such as the representation of rings by a family of quotients modulo prime ideals) we are given a topology on the base space (such as the hull-kernel topology in the case of rings and prime ideals). We would naturally prefer to obtain a field or sheaf over the given base with its given topology (and not necessarily with the field *-topology). The theorems about the canonical fields in $\$ 5$ tell us directly when sectional representation over a given topological base space is possible: This is the case if and only if the given topology is equal to or finer than the field 
*-topology. This, unfortunately, is rarely the case in the applications which arise in ring representation since the hull-kernel topology is generated by $S(a)=B \backslash h(a)$. In this section we will discuss a method which will allow us to amend the situation in an optimal way. In the algebraic situation we encountered this in $\$ 1$; it deserves to be called the method of topological localization and we maintain the name even for the somewhat more complicated analytical case. The essentials of this method have been presented in [20, p. 40 ff.], in a systematic fashion; but the present viewpoint is just a trifle different.

Localization. Let $(\pi, \mathfrak{U}), \pi: E \rightarrow B$ be a uniform field, and let $B$ be a subset of a topological space $C$. Let $\Sigma \subset \Gamma(\pi)$ be a full space of global sections. For any $c \in C$ we consider on $\Sigma$ the equivalence relation $R_{c}$ defined by $\sigma R_{c} \tau$ if and only if for each $U \in \mathfrak{U}$ there is a neighborhood $V$ of $c$ in $C$ such that $(\sigma(v), \tau(v)) \in U$ for $v \in V \cap B$. Let $\widetilde{E}_{c}=\Sigma / R_{c} \times\{c\}$ and define $\tilde{E}=\bigcup\left\{\tilde{E}_{c} \times\{b\} \mid c \in C\right\}$. The projection $\tilde{\pi}: \tilde{E} \rightarrow B$ is given by $\tilde{\pi}(\tilde{x}, c)=c$. For each section $\sigma \in \Sigma$ we obtain a serration $\tilde{\sigma}: C \rightarrow \widetilde{E}$ via the definition $\tilde{\sigma}(c)=R_{c}(\sigma)$. It is clear that the set $\tilde{\Sigma}=\{\tilde{\sigma} \mid \sigma \in \Sigma\}$ is full, i.e. that $\tilde{E}$ $=\bigcup\{\tilde{\sigma}(C) \mid c \in C\}$. Let now $U \in \mathfrak{U}$. For technical reasons only let us say that two sections $\sigma$ and $\tau$ of $\Sigma$ are $U$-close at $c \in C$ if there is a neighborhood $W$ of $c$ in $C$ such that $b \in B \cap W$ implies $(\sigma(b), \tau(b)) \in U$. We now define two subsets $U^{C}$ and $U_{1}^{C}$ of $\tilde{E} \vee \tilde{E}$ as follows:

(i) $U^{C}=\{(\tilde{x}, \tilde{y}) \in \widetilde{E} \vee \tilde{E} \mid \sigma$ and $\tau$ are $U$-close at $\tilde{\pi}(\tilde{x})=\tilde{\pi}(\tilde{y})$ for all $(\sigma, \tau) \in \tilde{x} \times \tilde{y}\}$,

(ii) $U_{1}^{C}=\{(\tilde{x}, \tilde{y}) \in \tilde{E} \vee \tilde{E} \mid \sigma$ and $\tau$ are $U$-close at $\tilde{\pi}(\tilde{x})=\tilde{\pi}(\tilde{y})$ for some $(\sigma, \tau) \in \tilde{x} \times \tilde{y}\}$.

Let us keep the following observations in mind:

LEMma 6.1. The sets $\left\{U^{C} \mid U \in \mathfrak{U}\right\}$ and $\left\{U_{1}^{C} \mid U \in \mathfrak{U}\right\}$ generate the same field uniformity $\tilde{\mathfrak{U}}$ for $\tilde{\pi}: \tilde{E} \rightarrow C$.

The proof rests on the fact that for a given $U \in \mathfrak{U}$ we may pick a $V \in \mathfrak{U}$ with $V=V^{-1}$ and $V V V \subseteq U$ and show that $V_{1}^{C} \subseteq U^{C} \subseteq U_{1}^{C}[20$, p. 41].

The following lemma is purely technical. It is, however, quite useful in the sequel.

Lemma 6.2. Let $W$ be an open set in $C$ and suppose that for two sections $\sigma, \tau \in \Sigma$ one has $W \cap B \subset\{b \in B \mid(\sigma(b), \tau(b)) \in U\}$ for a given $U \in \mathfrak{U}$. Then $(\tilde{\sigma}(c), \tilde{\tau}(c)) \in U_{1}^{c}$ for all $c \in W$. Conversely, if $(\tilde{\sigma}(c), \tilde{\tau}(c)) \in U^{c}$ for all $c \in W$, then $(\sigma(b), \tau(b)) \in U$ for $b \in B \cap W$.

Proof. (a) We recall $\tilde{\sigma}(c)=R_{c}(\sigma)$ and $\tilde{\tau}(c)=R_{c}(\tau)$. By the definition of $U_{1}^{C}$ we have $\left(R_{c}(\sigma), R_{c}(\tau)\right) \in U_{1}^{C}$ if there is a neighborhood $N$ of $c$ in $C$ such that $b \in N \cap B$ implies $(\sigma(b), \tau(b)) \in U$. Since we may take $N=W$ if $c \in W$, this condition is satisfied for $c \in W$. 
(b) For the converse, observe that $\left(R_{c}(\sigma), R_{c}(\tau)\right) \in U^{c}$ implies that $\sigma$ and $\tau$ are $U$-close at $c$. This immediately yields the converse.

Note that it follows from 6.2(a) that $\tilde{\sigma}$ and $\tilde{\tau}$ are $\beta$-related if $\sigma$ and $\tau$ are $\beta$-related (see discussion preceding 5.8).

According to 5.6 the set $\tilde{\Sigma}$ of serrations for $\tilde{\pi}$ inherits from $\tilde{\mathfrak{u}}$ a uniform structure, and we will from now on tacitly assume that $\Sigma$ is equipped with this uniform structure. We then also have the lemma:

LEMma 6.3. The function $\sigma \mapsto \tilde{\sigma}: \Sigma \rightarrow \tilde{\Sigma}$ is an isomorphism of uniform spaces. If $\Sigma$ is semiconnected, so is $\tilde{\Sigma}$.

Proof. Let $U \in \mathfrak{U}$. If $(\sigma(b), \tau(b)) \in U$ for all $b \in B$, then $(\tilde{\sigma}(c), \tilde{\tau}(c)) \in U_{1}^{c}$ for all $c \in C$ by Lemma 6.2 ; and if $(\tilde{\sigma}(c), \tilde{\tau}(c)) \in U^{c}$ for all $c \in C$, then $(\sigma(b), \tau(b))$ $\in U$ for all $b \in B$.

We have now associated with a space $\Sigma$ of global sections in a uniform field $(\pi, \mathfrak{U}), \pi: E \rightarrow B$ and with a space $C$ with $B \subset C$ a candidate for a new uniform field, namely $(\tilde{\pi}, \tilde{\mathfrak{U}}), \tilde{\pi}: \widetilde{E} \rightarrow C$ such that the given space $\Sigma$ is isomorphically injected into $\Gamma(\tilde{\pi})$. We are, however, lacking a field topology on $\widetilde{E}$. Such a field topology is secured in the following lemma:

LEMMA 6.4. As $\tilde{\sigma}$ runs through all sections of $\tilde{\Sigma}$ with $\tilde{\sigma}(\tilde{\pi}(\tilde{x}))=\tilde{x}, W$ through the collection of open sets of $C$, and $\tilde{U}$ through $\tilde{\mathfrak{u}}$, the resulting family of tubes $\tilde{U}(\tilde{\sigma} \mid W)$ is a basis for the neighborhood filter of $x$ relative to a field topology on $\widetilde{E}$ for $\tilde{\pi}$. This field-topology induces on the stalks $\widetilde{E}_{b}, b \in B$, the quotient topology associated with $\widetilde{E}_{b}=\Sigma / R_{b}$.

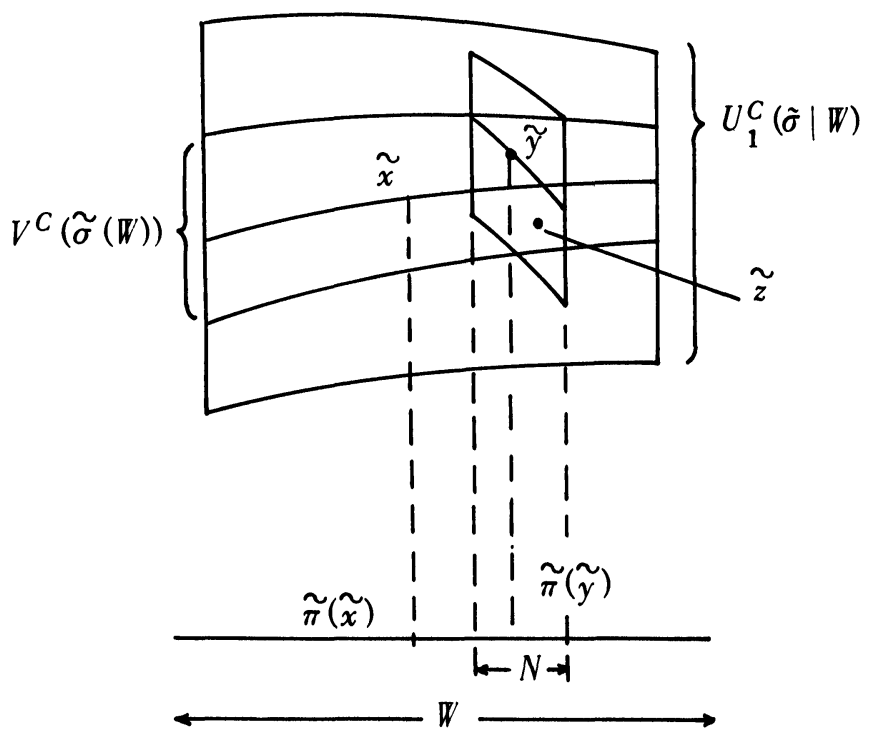

FIGURE 2 
Proof. Let $U \in \mathfrak{U}$ and pick $V \in \mathfrak{U}$ so that $V V \subseteq U$. Let $\tilde{y} \in V^{C}(\tilde{\sigma} \mid W)$. By the definition of $V^{C}$ this means that for all $\tau \in \tilde{y}$, the sections $\sigma$ and $\tau$ are $V$-close. Thus, for every such $\tau$ there is an open neighborhood $N \subseteq W$ of $\tilde{\pi}(\tilde{y})$ in $C$ such that $b \in N \cap B$ implies $(\sigma(b), \tau(b)) \in V$. We claim that $V^{C}(\tilde{\tau} \mid N)$ $\subseteq U_{1}^{C}(\tilde{\sigma} \mid W)$. Indeed let $\tilde{z} \in V^{C}(\tilde{\tau} \mid N)$. If $\rho \in \tilde{z}$, then $\tau$ and $\rho$ are $V$-close at $\tilde{\pi}(\tilde{z})$, i.e. there is an open neighborhood $M \subseteq N$ of $\tilde{\pi}(\tilde{z})$ in $C$ such that $b \in M \cap B$ implies $(\tau(b), \rho(b)) \in V$. Since $(\sigma(b), \tau(b)) \in V$ for all $b \in N \cap B$, we have $(\sigma(b), \rho(b)) \in V V \subseteq U$ for all $b \in N \cap B$. Thus by Lemma 6.2, $(\tilde{\sigma}(c), \tilde{\rho}(c)) \in U^{c}$ for all $c \in M$, and since $\tilde{\rho}(\tilde{\pi}(\tilde{z}))=\tilde{z}$ because of $\rho \in \tilde{z}$, the claim is established.

Thus we have associated with each $\tilde{x} \in \tilde{E}$ a filter basis $\mathfrak{B}(\tilde{x})$ of sets, and we have found for each $A \in \mathfrak{B}(\tilde{x})$ an $A^{\prime} \in \mathfrak{B}(\tilde{x})$ with $A^{\prime} \subseteq A$ such that for any $\tilde{y} \in A^{\prime}$ there is a $B \in \mathfrak{B}(\tilde{y})$ with $B \subset A$.

A set $Q$ in $\widetilde{E}$ is now called open if for each $\tilde{x} \in Q$ there is an $A \in \mathfrak{B}(\tilde{x})$ with $A \in Q$. This stipulation defines on $\tilde{E}$ a topology so that $\mathfrak{B}(\tilde{x})$ is a basis for the neighborhood filter of $\tilde{x}$.

Let now $b \in B$. A set $\widetilde{S} \subseteq E_{b}=\Sigma / R_{b} \times\{b\}$ is open in the quotient topology iff the full inverse image $S$ is open in $\Sigma$; this is the case iff for each $\sigma \in S$ there is a $U \in \mathfrak{U}$ such that $U(\sigma) \subseteq S$. But $\tau \in U(\sigma)$ means $(\sigma(b), \tau(b)) \in U$ for all $b \in B$, hence $(\tilde{\sigma}, \tilde{\tau}) \in U_{1}^{c}$ by 6.2 . But the point $\tilde{\sigma}(b) \in \tilde{S}$ has a basis of neighborhoods of the form $\left(U_{1}^{C}\right)(\tilde{\sigma}) \cap \widetilde{E}_{b}$ in $\widetilde{E}_{b}$ relative to the induced topology of $\widetilde{E}_{b}$. It follows that $S$ is open in $\Sigma$ iff $\widetilde{S}$ is open in $E_{b}$ relative to the induced topology.

Definition 6.5. If $(\pi, \mathfrak{U}), \pi: E \rightarrow B$ is a uniform field and $\Sigma$ a full set of cross sections, and if $B \subseteq C$ where $C$ is a topological space (whose topology does not necessarily induce the field star topology of $B$ ). Then the uniform field $(\tilde{\pi}, \tilde{\mathfrak{U}}), \tilde{\pi}: \tilde{E} \rightarrow C$ is called the field obtained by localization of $\Sigma$ over $C$. The isomorphism $\Sigma \rightarrow \tilde{\Sigma}$ is called the isomorphism induced by localization.

REMARK. The construction can be carried out for an arbitrary function $\phi: B \rightarrow C$ into a topological space instead of the inclusion function (see Dauns and HofmanN [20] and, for sheaves, KeImel [54]).

Let us observe at this point that after the canonical field associated with a group action this is the second instance of a canonical construction of a field.

The group case. Now let us immediately turn to the case of group actions recalling that for this case $\$ 5$ provided the best general results. Let us assume then that $(\pi, \mathfrak{U}), \pi: E \rightarrow B$ is the uniform field associated with a group action on the left of $E$ and a full set $\Sigma$ of serrations which are invariant under $G$ (see 1.15). It is not hard to see that the group $G$ with its action on $\Sigma$ permutes the cosets of the equivalence relation $R_{c}$ for any $c \in C$ (see [20, pp. 46, 47]) and that we therefore obtain an operation of $G$ on the left of $\tilde{E}$ by $g\left(b, R_{c}(\sigma)\right)=\left(b, R_{c}(g \sigma)\right)$. Moreover, $\tilde{\boldsymbol{\Sigma}}$ is clearly invariant under the action of $G$ on the serrations of $\tilde{\pi}$. We now observe that we have two field uni- 
formities for $\tilde{\pi}$ : Firstly the field uniformity $\tilde{\mathfrak{u}}$ constructed in 6.1 and secondly the field uniformity $\mathfrak{U}_{G}$ determined by the action of $G$ (see remarks preceding 5.14). The latter field uniformity has a basis of elements $U_{G}$ $=\{(\tilde{x}, \tilde{y}) \in \tilde{E} \vee \tilde{E} \mid \tilde{y} \in U \tilde{x}\}$, where $U$ ranges through the identity neighborhoods of 1 in $G$. Again we let $U^{\prime}=\{(x, y) \in E \wedge E \mid y \in U x\}$. Denote the neighborhood filter of $c$ in $C$ with $\mathfrak{B}(c)$. Then $\tau \in \tilde{y}$ and $(\tilde{x}, \tilde{y}) \in\left(U^{\prime}\right)_{1}^{C}$ means

(1) $(\exists \sigma \in \tilde{x})(\exists W \in \mathfrak{M}(\tilde{\pi}(\tilde{x})))(\forall b \in B) b \in W \Rightarrow(\exists u \in U) \tau(b)=u \sigma(b)$.

On the other hand, $(\tilde{x}, \tilde{y}) \in U_{G}$ means that there is a $u \in U$ with $\tilde{y}=u \tilde{x}$. Thus $\tau \in y$ and $(\tilde{x}, \tilde{y}) \in U_{G}$ means

$$
(\exists \sigma \in \tilde{x})(\exists u \in U)(\forall b \in B) \tau(b)=u \sigma(b) .
$$

Since trivially (2) implies (1) we have $\tilde{\mathfrak{U}} \subseteq \mathfrak{U}_{G}$.

We now concentrate our attention on canonical fields.

LEMma 6.6. Let $(\pi, \mathfrak{U}), \pi: E \rightarrow B$ be the canonical uniform field associated with $G$ and $\left\{K_{b} \mid b \in B\right\}$, and suppose that $B \subset C$, where $C$ is a given topological space. (Recall that we do not assume that $B$ is a subspace!) For $c \in C$ let $\mathfrak{B}(c)$ again be the neighborhood filter of $c$ in $C$. Then $\tilde{K}_{c}=$ $\bigcap_{U} \bigcup_{W} \bigcap_{b} U K_{b}, b \in W \cap B, W \in \mathfrak{B}(c), U \in \mathbb{G}$ (the neighborhood filter of the identity of $G$ ) is a subgroup of $G$.

We consider three fields:

(a) the uniform field $(\underline{\tilde{\pi}}, \mathfrak{\mathbb { U }}), \tilde{\pi}: \tilde{E} \rightarrow C$ obtained by localization by $\hat{G}$ over $C$,

(b) the canonical field $\left(\pi^{\#}, \mathfrak{U}_{G}\right), \pi^{\#}: E^{\#} \rightarrow C^{\#}$ associated with $G$ and $\left\{\tilde{K}_{c} \mid c \in C\right\}$, where $C^{\#}$ is the underlying set of $C$ with the field star topology,

(c) the field $\left(\pi^{\#}, \mathfrak{U}_{G}\right), \pi^{\#}: E^{\# \#} \rightarrow C^{\# \#}$ where $E^{\# \#}$ has the same underlying set as $E^{\#}$ with the topology generated by the basic sets $\pi^{-1}(W) \cap V, W$ open in $C, V$ open in $E^{\#}$, and where the topology of $C^{\# \#}$ is the least upper bound of the topologies of $C^{\#}$ and $C$.

Then we have a commutative diagram of continuous functions

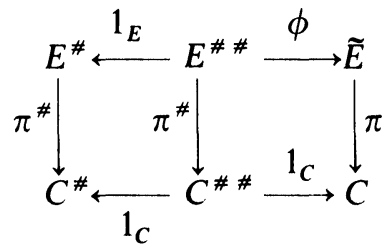

with $\phi(g \widetilde{G}, c)=\left(R_{c}(\hat{g}), c\right)$. In fact $\phi$ is continuous (in fact uniformly on stalks) and bijective. On each stalk, $\phi$ induces an isomorphism if $\hat{G} \subseteq \Gamma(\pi)$ is isomorphic (as uniform space) to $G$ under the Gelfand morphism.

Proof. Let $c \in C$. The relation $R_{c}$ identifies $\hat{g}$ and $\hat{h}$ in $\hat{G}$ if for each identity neighborhood $U$ of $G$ there is a $W \in \mathfrak{M}(c)$ such that $b \in W \cap B$ 
implies $(g(b), h(b)) \in U^{\prime}$, which means exactly $g \in U h K_{b}$. Thus $\hat{g} R_{c} \hat{h}$ iff $g \in \bigcap\left\{U h K_{b} \mid b \in W \cap B\right\}$ for a suitable neighborhood $W$ of $c$ in $C$. In particular, $R_{c}(\hat{\mathbf{1}})=\{\hat{\mathfrak{g}} \in \hat{G} \mid$ for any $U \in \mathfrak{G}$ there is a $W \in \mathfrak{B}(c)$ with $\left.g \in \bigcap\left\{U K_{b} \mid b \in W \cap B\right\}=\bigcap_{U} \bigcup_{W} \bigcap_{b \in W \cap B} U K_{b}\right\}$. In order to see that $\widetilde{G}_{c}=\left\{g \in G \mid \hat{g} \in R_{c}(1)\right\}$ is, in fact, a subgroup, we observe that $G$ permutes the cosets $R_{c}(g)$ transitively under the operation of $G$ on the left of $\hat{G}$, since $G$ is transitive on $\hat{G}$ and respects $R_{c}$. Thus $R_{c}(\hat{1})$ is the orbit of the subgroup fixing $R_{c}(\hat{1})$ as a whole. This subgroup is precisely the set of $g$ with $\hat{g} R_{c} \hat{1}$, i.e. is $\widetilde{K}_{c}$. The function $g \mapsto R_{c}(\hat{g}): G \rightarrow \hat{G} / R_{c} \cong \widetilde{E}_{c}$ factors through the space $G / \widetilde{K}_{c}$ of cosets $g \widetilde{K}_{c}$. We thus define a bijective function $\phi: E^{\#} \rightarrow \widetilde{E}$ by $\phi\left(g \tilde{K}_{c}, c\right)=\left(R_{c}(\hat{g}), c\right)$. Then the diagram (1) commutes. Since $\tilde{E}_{c}$ inherits the quotient topology of $\hat{G} / R_{c}$ by 6.4 , the function $\phi_{c}$ in the diagram

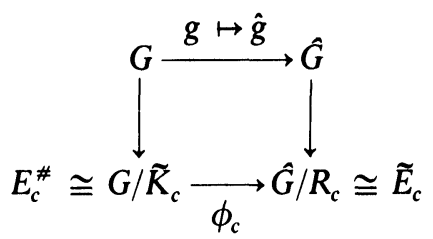

is continuous.

We identify $E$ with $E^{\# \#}$ qua sets and write $\tilde{\mathfrak{U}}$ for the field uniformity of $E$ transported to $E^{\# \#}$. After a remark preceding (6.6) we then have $\tilde{\mathfrak{U}} \subseteq \mathfrak{U}_{G}$. This implies the continuity of $\phi$. The remainder is clear.

Note that for each $g \in \widetilde{K}_{c}$ and each identity neighborhood $U$ of $G$ we have a neighborhood $W$ of $c$ such that $g \in \bigcap\left\{U K_{b} \mid b \in W \cap B\right\}$ which is contained in $U K_{c}$ if $c \in B$. Hence $\tilde{K}_{c} \subseteq \bigcap_{U} U K_{c}=\bar{K}_{c}$ if $c \in B$.

In the present situation let us describe explicitly the basic sets of $\tilde{\mathfrak{U}}$. If $U \in \mathbb{G}$, then the sets $\left(U^{\prime}\right)^{C}$ contain exactly the pairs $\left(g \tilde{K}_{c}, c\right),\left(h \widetilde{K}_{c}, c\right)$ such that for all $g^{\prime} \in g \widetilde{K}_{c}$ and $h^{\prime} \in h \widetilde{K}_{c}$ one has a $W \in \mathfrak{B}(c)$ such that $b \in W \cap B$ implies $h^{\prime} \in U g^{\prime} K_{b}$.

It follows that $(\widetilde{E}, \tilde{\mathfrak{U}})$ is a uniformly homogeneous uniform field. If all $K_{b}$ are normal, then the $\widetilde{K}_{c}$ are normal (as follows rather immediately from the definition of $\left.\widetilde{K}_{c}\right)$ and we have the following simpler description: $\left(U^{\prime}\right)^{C}$ $=\left\{\left(\left(g \tilde{K}_{c}, c\right),\left(h \widetilde{K}_{c}, c\right)\right) \mid\right.$ for all $g \in K_{c}$ there is a neighborhood $W$ of $c$ in $C$ such that $b \in B \cap W$ implies $\left.h \in U K_{b} \tilde{g}\right\}$.

Moreover, $(\tilde{\pi}, \tilde{\mathfrak{U}}), \tilde{\pi}: \widetilde{E} \rightarrow C$ is a uniform field of topological groups.

The localization theorem. Let us formulate the principal results in the following theorem:

THEOREM 6.7 (The localization theorem). Let $G$ be an SIN-group and $\left\{K_{b} \mid b \in B\right\}$ a family of normal subgroups. Let $C$ be a topological space containing $B$ as a subset and let $(\pi, \mathfrak{u}), \pi: E \rightarrow B$ be the canonical uniform field of topological groups associated with $G$ and $\left\{K_{b} \mid b \in B\right\}$. Then there is a family of normal subgroups $\widetilde{K}_{c}=\bigcap_{B} \bigcup_{W} \bigcap_{b} U K_{b}$, where $U$ ranges through the 
identity neighborhoods of $G, W$ through the neighborhoods of $c$ in $C$ and $b$ through $B \cap W$, and for the surjective function $\tilde{\pi}: \tilde{E} \rightarrow C, \tilde{E}=\left\{G / \widetilde{K}_{b}\right.$ $\times\{c\} \mid c \in C\}$ there is a field uniformity $\tilde{\mathbb{U}}$ and a field topology on $\tilde{E}$ such that $(\tilde{\pi}, \tilde{\mathfrak{U}})$ is a uniform field of topological groups in which the stalks $G / \widetilde{K}_{c}$ have an induced topology which agrees with the quotient topology if the Gelfand morphism maps $G$ isomorphically onto $\hat{G} \subseteq \Gamma(\pi)$. If $b \in B$ then $\widetilde{K}_{b} \subseteq \bar{K}_{b}$. The function $\hat{\mathrm{g}} \mapsto \tilde{\mathrm{g}}: \hat{G} \rightarrow \tilde{G} \subseteq \Gamma(\pi)$, with $\tilde{\mathrm{g}}(c)=\left(g \tilde{K}_{c}, c\right)$ is an isomorphism of topological groups. If $\hat{G} \subseteq \Gamma^{b}(\pi)$, then $\widetilde{G} \subseteq \Gamma^{b}(\tilde{\pi})$.

The field uniformity $\mathfrak{U}_{G}$ which is defined on $E$ by the structure of a canonical field associated with $G$ and the family $\left\{\widetilde{K}_{c} \mid c \in C\right\}$ of normal subgroups contains $\tilde{\mathfrak{U}}$. If $G$ is discrete then $(\tilde{\pi}, \tilde{\mathfrak{U}})$ is this canonical field and is, in fact, a sheaf. In this case, $\widetilde{K}_{c}=\bigcup_{W} \bigcap_{b} K_{b}, W$ ranging through the neighborhoods of $c$ in $C$ and $b$ through $B \cap W$.

We will shortly say that $(\tilde{\pi}, \tilde{\mathfrak{U}})$ is the canonical field for $G$ and $\left\{K_{b} \mid b \in B\right\}$ obtained from localization over $C$.

REMARK. We leave open the question of which circumstances would generally enforce $\mathfrak{U}_{G}=\widetilde{\mathfrak{U}}$. The conditions given in [20, p. 48] do not seem to be accurate.

If the two uniformities are the same, then the field star topology induced on the set $C$ by the canonical field is coarser than $C$, but otherwise this is not clear. Note that for discrete $G$, i.e. in the sheaf case, we do have this information. Note that we have recovered and in fact generalized to the analytical case the Construction 1.4.

The case that $C$ induces on $B$ the field star topology of the field $(\pi, \mathfrak{u})$ and that $\mathfrak{U}$ is a separated uniform structure is particularly easy to visualize (see [20, p. $42 \mathrm{ff}$.$] ).$

Proposition 6.8. If, under the circumstances of Theorem 6.7, the topology which is induced on $B$ by $C$ and the field star topology on $B$ given by the canonical field $(\pi, \mathfrak{U})$ agree and if $\mathfrak{U}$ is separated, then there is a commutative diagram

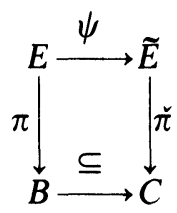

in which the horizontal maps are embeddings, and the field uniformity $\mathfrak{U}$ agrees with the one induced by $\tilde{\mathfrak{U}}$ via $\psi$.

RemarK. It is easy to note that in a point $b \in B$ we have $\widetilde{K}_{b}=K_{b}$ if $\mathfrak{U}$ is separated and every field star neighborhood of $b$ in $B$ is of the form $B \cap W$ with a $C$-neighborhood $W$ of $b$. 
§7. Fields of topological rings and modules. In the last two sections we produced canonical fields which allow us to represent given topological groups as groups of global sections, and in view of the results concerning localization we can do this even if we prescribe a topology on the base space. We have no means so far to ascertain that the image under the Gelfand morphism is large in $\Gamma(\pi)$. For topological groups in general no methods are known which would contribute to this question. However, for topological modules, the situation is more satisfactory. In order to discuss sectional representation of modules adequately we have to talk about fields of topological rings.

Fields of rings.

DEFINITION 7.1. A uniform field of rings $(\rho, \mathfrak{B}), \rho: F \rightarrow B$ is a uniform field of abelian groups (written additively) for which a continuous function $(x, y) \mapsto x y: F \vee F \rightarrow F$ is defined relative to which each stalk $F_{b}=\rho^{-1}(b)$ becomes a topological ring. If $(\pi, \mathfrak{U}), \pi: E \rightarrow B$ is a uniform field of topological abelian groups (written additively), then $(\pi, \mathfrak{U})$ is called a $(\rho, \mathfrak{B})$ module (shorter: $\pi$ is a $\rho$-module) if there is a continuous bilinear map $(r, x) \mapsto r x: F \vee E \rightarrow E$ (where, of course $F \vee E=\{(r, x) \in F \times E \mid \rho(r)$ $=\pi(x)\})$ relative to which each stalk $E_{b}=\pi^{-1}(b)$ becomes a topological $R_{b}$-module.

One immediately deduces

LEMMA 7.2. If $(\rho, \mathfrak{B}), \rho: F \rightarrow B$ is a uniform field of rings then $\Gamma(\rho)$ is an additive topological group and a ring. If $(\pi, \mathfrak{U})$ is a $(\rho, \mathfrak{B})$-module, then $\Gamma(\pi)$ is a $\Gamma(\rho)$-module.

It must be pointed out that neither $\Gamma(\rho)$ need be a topological ring nor $\Gamma(\pi)$ a topological $\Gamma(\rho)$-module.

This is not even the case in the simplest cases of constant fields $E=K \times B$ where $K$ is a topological ring, $B$ a noncompact space and $\pi: E \rightarrow B$ the projection, and where the field uniformity on $E$ is the obvious one constructed from the uniformity of the additive group of $K$. Then $\Gamma(\pi)$ is isomorphic to the ring of all continuous functions from $B$ into $K$, and this ring is not topological if $B$ is not compact.

It is also generally not the case that the closed additive subgroup $\Gamma^{b}(\pi)$ of all bounded sections in $\Gamma(\pi)$ is even a subring. To investigate this question further, we need the concept of boundedness in a topological group. A subset $C$ of a topological group $G$ is bounded if for every identity neighborhood $U$ of $G$ there is a natural number $n$ such that $C \subseteq U^{n}$. If $(\pi, \mathfrak{u}), \pi: E \rightarrow B$ is a uniform field of topological groups, then a subset $C \subseteq E$ is bounded if for any $U \in \mathfrak{U}$ there is a natural number $n$ so that $C \subseteq(U(1))^{n}$. A topological group $G$ is locally bounded if the neighborhood filter of the identity has a basis of bounded sets. A uniform field of topological groups is locally 
bounded if its field uniformity has a basis of sets $U$ such that $U(1)$ is bounded. The canonical field associated with a locally bounded group and a family of normal subgroups is locally bounded.

Now let $G, H$, and $K$ be additively written topological abelian groups. Let $f: G \times H \rightarrow K$ be a continuous bilinear map. If $\left\{G_{b}\right\},\left\{H_{b}\right\},\left\{K_{b}\right\}, b \in B$, are families of subgroups of $G, H$, and $K$, respectively, we will call them compatible with $f$ if $f\left(G_{b} \times H_{b}\right) \subseteq K_{b}$. In this case $f$ induces a continuous bilinear map $f_{b}: G / G_{b} \times H / H_{b} \rightarrow K / K_{b}$ via $f_{b}\left(g+G_{b}, h+H_{b}\right)=f(g, h)$ $+K_{b}$.

Let now $\left(\pi_{G}, \mathfrak{U}_{G}\right), \pi_{G}: E_{G} \rightarrow B$, etc., be the canonical fields associated with $G, H, K$ and the families of subgroups introduced above. The $f_{b}$ induce a bilinear function $\bar{f}: E_{G} \vee E_{H} \rightarrow E_{K}$. This function is continuous $\left[20\right.$, p. 60]. Therefore there is a bilinear function $\tilde{f}: \Gamma\left(\pi_{G}\right) \times \Gamma\left(\pi_{H}\right) \rightarrow \Gamma\left(\pi_{K}\right)$ given by $\tilde{f}(\sigma, \tau)(b)=\bar{f}(\sigma(b), \tau(b))=f_{b}(\sigma(b), \tau(b))$. If $B$ is compact, then $\tilde{f}$ is continuous; in any case, if $f$ is uniformly continuous on bounded sets, then so are $\bar{f}$ and $\tilde{f}$, provided that $G$ and $H$ are locally bounded [20, p. 60]. In this case $f\left(\Gamma^{b}\left(\pi_{G}\right) \times \Gamma^{b}\left(\pi_{H}\right)\right) \subseteq \Gamma^{b}\left(\pi_{K}\right)$, since $f$ maps bounded sets into bounded sets. If $G, H$ and $K$ are connected then we have $\hat{G} \subseteq \Gamma^{b}\left(\pi_{G}\right)$ etc., by 5.20.3 There are some obvious corollaries:

Proposition 7.3. Let $R$ be a topological ring and $\left\{R_{b} \mid b \in B\right\}$ a family of ideals. Then the uniform field $(\pi, \mathfrak{U}), \pi: E \rightarrow B$ associated with this data is a field of topological rings, and if multiplication in $R$ is uniformly continuous on bounded sets then so is the multiplication of $E$. Moreover, $\Gamma(\pi)$ is a ring on a topological abelian group whose multiplication is uniformly continuous on bounded sets if $R$ is locally bounded and its multiplication has this property. Also, $\Gamma^{b}(\pi)$ is a subring. If $B$ is compact, then $\Gamma(\pi)$ is a topological ring, regardless. If $R$ is connected, then $\hat{R} \subseteq \Gamma^{b}(\pi)$. (Recall that the quasicompactness of $B$ implies $\Gamma^{b}(\pi)=\Gamma(\pi)$ by 5.9) $[20, p .62]$.

Proposition 7.4. Let $R$ be a topological ring and $M$ a topological $R$ module such that scalar multiplication is uniformly continuous on bounded sets. Let $\left\{M_{b} \mid b \in B\right\}$ be a family of submodules of $A$ and $(\pi, \mathfrak{U}), \pi: E \rightarrow B$ the associated canonical uniform field of topological groups. Then there is a scalar multiplication $\left(r, a+M_{b}\right) \mapsto r a+M_{b}: R \times E \rightarrow E$ which is continuous and is uniformly continuous on bounded sets. There is a scalar multiplication $(f, \sigma) \mapsto f \sigma: C(B, R) \times \Gamma(\pi)$ given by $(f \sigma)(b)=f(b) \sigma(b)$, which is uniformly continuous on bounded sets, if $R$ and $A$ are locally bounded. If $B$ is compact, then scalar multiplication is continuous. The subgroup $\Gamma^{b}(\pi)$ is a $C^{b}(B, R)$ submodule, where $C^{b}$ of course denotes the ring of bounded functions.

Proposition 7.5. Let $R$ be a topological ring and $\left\{I_{b} \mid b \in B\right\}$ a family of ideals. Let $M$ be a topological $R$-module and $M_{b}$ for each $b \in B$ a submodule containing all linear combinations of elements $r m$ with $r \in I_{b}$ and $m \in M$. 
Let $\left(\pi_{R}, \mathfrak{U}_{R}\right), \pi_{R}: E_{R} \rightarrow B$ be the uniform field of topological rings associated with $R$ and $\left\{I_{b} \mid b \in B\right\}$ and $\left(\pi_{M}, \mathfrak{U}_{M}\right), \pi_{M}: E_{M} \rightarrow B$ the uniform field of abelian groups associated with $M$ and $\left\{M_{b} \mid b \in B\right\}$. Then $\pi_{M}$ is a topological $\pi_{R^{-}}$ module and if $B$ is compact, then $\Gamma\left(\pi_{A}\right)$ is a topological $\Gamma\left(\pi_{R}\right)$-module. If the scalar multiplication $R \times M \rightarrow M$ is uniformly continuous on bounded sets, then so is the scalar multiplication $\Gamma\left(\pi_{R}\right) \times \Gamma\left(\pi_{M}\right) \rightarrow \Gamma\left(\pi_{M}\right)$. The subgroup $\Gamma^{b}\left(\pi_{M}\right)$ is a $\Gamma^{b}\left(\pi_{R}\right)$-submodule.

Partitions of identity. Now we are ready to introduce partitions of identity. The following results exemplify the methods; unfortunately, in order to cover all cases we have to introduce a rather complicated concept which in the discrete case in essence reduces to the idea of partition of identity which we encountered in $\$ 1$.

DEFINITION 7.6. Let $(\pi, \mathfrak{U})$ be a uniform field of abelian groups. We say that a subgroup $M \subseteq \Gamma(\pi)$ has approximate bounded partitions of unity if for any $m \in M$, any $U \in \mathfrak{U}$ and any finite cover $C$ of $B$, there is a bounded subset $X \subseteq M$ and a family $\left\{e_{W} \mid W \in C\right\}$ of elements of $X$ such that (a) $e_{W}(b)=\mathcal{O}(b)$ for $b \notin W$ and (b) $\left(\sum e_{W}-m\right)(b) \in U(0)$ for all $b \in B$.

The significance of the concept lies in the following result which guarantees that a subgroup $M$ of $\Gamma(\pi)$ has a large supply of sections. Since we discussed the algebraic (sheaf) case extensively in $\S \S 1$ and 2, we restrict our attention to the analytic case.

Before we formulate the theorem we note that we define a topological space to have dimension $n$ if every compact subset of $K$ has Lebesgue covering dimension $\leqq n$ and if $n$ is the smallest natural number with this property.

THEOREM 7.7 (The richness theorem for sections). Let $K$ be a locally compact finite dimensional topological ring and $(\pi, \mathfrak{U}), \pi: E \rightarrow B$ a uniform field of topological $K$-algebras; then $\Gamma^{b}(\pi)$ is a $C^{b}(B, K)$-module via $(f \cdot \sigma)(b)$ $=f(b) \cdot \sigma(b)$, where $C^{b}(B, K)$ is the K-algebra of all continuous bounded functions $f: B \rightarrow K$. Suppose that $M$ is a closed $K$-submodule of $\Gamma^{b}(\pi)$ which has approximate bounded partitions of identity. Then $M$ is a $C^{b}(B, K)$ module.

Proof. Let $f \in C^{b}(B, K)$ and $m \in M$. We have to show that $f \cdot m \in M$; since $M$ is closed in $\Gamma^{b}(\pi)$ it suffices to find an $m^{\prime} \in M$ as close as we wish to $f \cdot m$ in $\Gamma^{b}(\pi)$. Let $V$ be a zero neighborhood in $K$. The set $f(B)^{-}$is closed and bounded in $K$, hence compact, and so is of dimension $\leqq n$ where $n=\operatorname{dim} K$. A finite number of translates of the open set $V$ then covers the compact subspace $f(B)^{-}$, and for reasons of Lebesgue covering dimension, this cover has a finite refinement such that at most $n+1$ sets in the cover overlap. Let $C$ be the finite cover of all $W=f^{-1}\left(W_{0}\right)$ where $W_{0}$ runs through the elements of the cover we have just constructed. 
For each $W \in C$ we have $W=f^{-1}\left(W_{0}\right)$ with $W \subseteq r_{W}+V$ for some $r_{W} \in K$. Then $f(c)-r_{W} \in V$ for $c \in W$. Now we take an element $U \in \mathfrak{U}$ and find, by 7.6, a bounded set $X$ in $M$ and elements $e_{W} \in X, W \in C$ such that

(a) $e_{W}(b)=\mathcal{C}(b)$ for $b \notin W$,

(b) $\left(m-\sum e_{W}\right)(b) \in U(0)$ for all $b \in B$.

Since $M$ is a $K$-submodule by hypothesis, then $m^{\prime}=\sum r_{W} \cdot e_{W}$ is an element of $M$. Then we have $f \cdot m-m^{\prime}=f \cdot m-\sum r_{W} \cdot e_{W}=f \cdot\left(m-\sum e_{W}\right)$ $+\sum\left(f-r_{W}\right) \cdot e_{W}$.

The first summand is contained in $f \cdot U(0)$ which can be made as small as we want since $f$ is bounded.

Because of condition (a) above, the sum $\left(\sum\left(f-r_{W} \cdot e_{W}\right)\right)(b)$ need only to be extended over those $W \in C$ for which $b \in W$. But since at most $n+1$ elements of $C$ overlap, then this sum has at most $n+1$ terms. Moreover, $\left(f-r_{W}\right)(c) \in V$ for all $c \in W$. Since $e_{W} \in X$ and since $n$ is fixed and $X$ bounded, $\sum\left(f-r_{W}\right) \cdot e_{W}$ may be made as small as we wish. This finishes the proof.

Some remarks should illuminate the rather technical circumstances of the richness theorem.

The existence of approximate bounded partitions of unity is guaranteed in the very relevant case of certain operator algebras as we shall see. Note that if $K$ is a division ring, then the dimension hypothesis is automatically satisfied, since a locally compact vector space over a nondiscrete field is finite dimensional. In fact the topological dimensions in this case are $0,1,2$, or 4 .

Stone Weierstrass theorem. The preceding richness theorem has as a consequence a theorem of Weierstrass type in which the field $(\pi, \mathfrak{U}) \pi: E$ $\rightarrow B$ is, in fact, a field of locally convex vector spaces. Let us explain what this means in the following definition:

Definition 7.8. A uniform field $(\pi, \mathfrak{U}), \pi: E \rightarrow B$ of topological abelian groups is called a locally convex field if all stalks $E_{b}$ are real vector spaces, the scalar multiplication $R \times E \rightarrow E$ is continuous, and if $\mathfrak{U}$ has a basis of elements $U$ such that $U(0) \cap E_{b}$ is convex for every $b \in B$. In this case $U$ is called a convex entourage. (This definition extends to complex scalars in an obvious fashion.)

A locally convex field is a field of Banach spaces if there is a function \|\|$: E \rightarrow[0, \infty$ [ which induces on each stalk a Banach space norm and is such that the sets $U_{\varepsilon}=\{(x, y) \in E \vee E \mid\|x-y\|<\varepsilon\}$ form a basis for the field uniform structure.

If all stalks in a locally convex field (field of Banach spaces) are topological algebras (Banach algebras) and the multiplication $E \vee E \rightarrow E$ is continuous, then $(\pi, \mathfrak{l})$ is called a field of real (resp. complex) topological algebras (Banach algebras). 
Fields of Banach spaces and algebras are the most important in the analytical applications. The space of bounded sections $\Gamma^{b}(\pi)$ is then a Banach space (algebra) relative to the sup-norm $\|\sigma\|=\sup \{\|\sigma(b)\| \mid b \in B\}$. For each $\sigma \in \Gamma^{b}(\pi)$ the function $b \mapsto\|\sigma(b)\|$ is upper semicontinuous [20, pp. 11, $115 \mathrm{ff}$.$] .$

We are now in a position to formulate a general Stone Weierstrass theorem which should be used in conjunction with the richness Theorem 7.7 and which shows in effect, the true significance of 7.7. Let us say that a space $X$ is quasi-completely regular if for any $x \in X$, and any closed $Y \subseteq X$ with $x \notin Y$ there is a continuous function $f: X \rightarrow[0,1]$ with $f(x)=0, f(Y)$ $=\{1\}$.

TheOREM 7.9 (Stone Weierstrass theorem). Let $(\pi, \mathfrak{U}), \pi: E \rightarrow B$ be a uniform locally convex field over a quasi-compact quasi-completely regular space. Consider $\Gamma(\pi)$ as a $C(B)$-module under $(f \cdot \sigma)(b)=f(b) \sigma(b)$, where $C(B)$ is the $C^{*}$-algebra of all real or complex valued functions on $B$. Suppose that $M \subseteq \Gamma(\pi)$ is a closed $C(B)$-submodule with the property that the set $M(b)=\{m(b) \mid m \in M\}$ is dense in the stalk $E_{b}$ for all $b$. Then $M=\Gamma(\pi)$.

Proof. Let $\sigma \in \Gamma(\pi)$. For a given $U \in \mathfrak{U}$ and for any $b \in B$ we find elements $m_{b} \in M$ such that $\sigma(b)-m_{b}(b) \in U(0)$, and by continuity we may even assume that we find a neighborhood $W_{b}$ of $b$ in $B$ such that $\sigma(c)-m_{b}(c)$ $\in U(0)$ for $c \in W_{b}$. By quasi-compactness of $B$ we find a finite subcover $C$ in $\left\{W_{b} \mid b \in B\right\}$. Write $m_{W}=m_{b}$ if $W=W_{b}$. Since $B$ is quasi-completely regular we find functions $f_{W} \in C(B, \boldsymbol{R})$ such that $0 \leq f_{W}$ and $f_{W}(b)=0$ for $b \notin W$ and $\sum f_{W}=1, W \in C$. Since $M$ is a $C(B)$-module, the element $m=\sum f_{W} \cdot m_{W}$ is in $M$. Now $\sigma-m=\sum f_{W} \cdot\left(\sigma-m_{W}\right)$. We may assume that $U$ is a convex entourage. Then $\sum f_{W}(b) \cdot\left(\sigma-m_{W}\right)(b)$ is a convex combination of the elements $\left(\sigma-m_{W}\right)(b)$ which are in $U(0) \cap \pi^{-1}(b)$ for all $W$ for which $b \in W$, i.e. for which $f_{W}(b) \neq U$. Hence $\sigma-m \in U(0)$. Since $M$ is closed this finishes the proof.

The richness and the Stone Weierstrass theorems together then have the following important consequence, which will be used in the proof of a noncommutative Gelfand Naimark theorem for $C^{*}$-algebras.

Corollary 7.10. Let $(\pi, \mathfrak{U}), \pi: E \rightarrow B$ be a uniform field of locally convex spaces over a quasi-compact and quasi-completely regular base space B. If $M$ is a closed subvector space of $\Gamma(\pi)$ such that

(1) $M$ has bounded approximate partitions of unity,

(2) $M(b)$ is dense in the stalk $E_{b}$ for each $b \in B$.

Then $M=\Gamma(\pi)$.

$\S 8$. Representations of topological rings by sections in fields. In $\S \S 1-4$ we gave a survey of sectional representation of discrete rings, $R$. Here comes the topological (analytical) version of the theory. 
Let $R$ be a locally convex topological algebra over the reals or complexes. The most significant applications up to date concern the case that $R$ is a $C^{*}$-algebra. As far as I know there has been no interest in prime ideal spaces $B$ other than the space of all primitive ideals, or the space of all maximal [respectively, maximal modular] ideals.

Localization for topological rings. We let $\pi: E \rightarrow B_{0}$ be the canonical sheaf of topological rings associated with $R$ and $B$, where $B_{0}$ is the set $B$ with the field star topology. We then obtain a Gelfand morphism $R \rightarrow \Gamma^{b}(\pi)$ whose kernel is $\bigcap B$; if $R$ is a semisimple algebra (which is automatic if $R$ is a $C^{*}$-algebra and $B=\operatorname{Prim} R$ is the space of all primitive ideals), then the Gelfand morphism is injective.

We are looking for a condition which would ensure that the Gelfand morphism is an isomorphism of topological algebras onto its image. The topology on $R$ which makes the Gelfand morphism an isomorphism has as basic zero neighborhoods the sets $\bigcap\{U+I \mid I \in B\}, U$ ranging through the zero neighborhoods of the given topology of $R$. In order that the Gelfand morphism is an isomorphism of topological algebras onto its image it is therefore necessary and sufficient that

(I) for each zero neighborhood $U$ in $R$ there exists a zero neighborhood $V$ such that $\bigcap\{V+I \mid I \in B\} \subseteq U$.

Following 6.7 we now produce the field $(\tilde{\pi}, \tilde{\mathfrak{U}}), \tilde{\pi}: \widetilde{E} \rightarrow B$ of topological algebras obtained from $\pi: E \rightarrow B_{0}$ by localization of $\hat{R}$ over $B$ with the hull-kernel topology. Then the stalks are algebraically the quotient algebras $A / \tilde{I}$ where

$$
\tilde{I}=\bigcap_{U} \bigcup_{W} \bigcap_{J}(U+J), \quad \begin{aligned}
& U \in W, \quad W \text { a neighborhood of } I \text { in } B, \\
& U \text { a zero neighborhood of } A .
\end{aligned}
$$

Indeed it was shown in 6.7, that $\tilde{I}$ is a topological group; that $\tilde{I}$ is stable under multiplication from the right is clear from the fact that $I$ is an ideal; if $a \in R$ then for each $U$ there is a $V$ with $a V \subseteq U$, hence $a \tilde{I}=$ $\bigcap_{V} \bigcup_{W} \bigcap_{J} a(V+J) \subseteq \bigcup_{W} \bigcap_{J}(U+J)$, and since this holds for all $U$ we have $a \tilde{I} \subseteq \tilde{I}$. The topology induced on $\widetilde{E}_{b}$ is generally coarser than the topology corresponding to the quotient topology, but agrees with it under the hypothesis (I) (6.7). If $a \in(\tilde{I})^{-}$, then for each zero neighborhood $U$ of $A$ there is an $a_{0} \in \tilde{I}$ and $a \in a_{0}+U$ so there is a neighborhood $W$ of $I$ with $a_{0} \in c+U$ for $c \in W$; hence $a \in c+U+U$ for $c \in W$. It follows that $a \in \tilde{I}$, therefore $\tilde{I}$ is closed.

By 6.7 we have $\tilde{c} \subseteq \bigcap_{U}(U+c)=\bar{c}$. We therefore require

(II) all $I \in B$ are closed.

$C^{*}$-algebras. This condition is automatic in $C^{*}$-algebras for $B \subseteq \operatorname{Prim} A$. Under these circumstances we have $\tilde{I} \subseteq I$. If for some open $V \subseteq B$ we have $a \in \bigcap\{\tilde{I} \mid I \in V\}$, then for each $I \in V$ and each $U$ there is a $W \subseteq V$ so 
that $a \in \bigcap\{U+J \mid J \in W\} \subseteq U+I$ so $a \in \bar{I}=I$, since $U$ was arbitrary. Conversely if $a \in \bigcap V$ then $a \in \bigcap\{U+J \mid J \in V\}$ for all $U$, hence $a \in$ $\bigcup_{W} \bigcap\{U+J \mid J \in V\}$ for all $I \in V$ where $W$ ranges through the neighborhoods of $I$. Thus $a \in \bigcap\{\tilde{I} \mid I \in V\}$. We therefore have the analogue of 1.5:

Lemma 8.1. For each open $W$ of $B$ we have $\bigcap\{\tilde{I} \mid I \in W\}=\bigcap W$.

We now have a Gelfand morphism $r \mapsto \tilde{r}: R \rightarrow \Gamma^{b}(\tilde{\pi})$ which is an isomorphism onto its image. If $R$ is complete, then so is the image and the image therefore is closed in $\Gamma^{b}(\pi)$.

We now will discuss the structure of $(\tilde{\pi}, \tilde{\mathfrak{u}})$ as a field of $C^{*}$-algebras. All stalks $R / \tilde{I}$ are clearly involutive topological algebras, and each has a quotient norm. This norm defines the field uniformity of the canonical field of $C^{*}$-algebras $\left(\tilde{\pi}, \mathfrak{U}_{R}\right): \tilde{\pi}: E^{\#} \rightarrow B^{\#}$ associated with $R$ and $\{\tilde{I} \mid I \in B\}$.

Now we consider the function ||$: \tilde{E} \rightarrow[0, \infty)$ given by $|a+\tilde{I}|$ $=\inf _{W} \sup _{J}\|a+J\|$, where $J \in W$ and $W$ ranges over the hull-kernel neighborhoods of $I$, where $\|a+J\|=\|\hat{a}(J)\|$ is the quotient norm in $A / J$.

Clearly $a \mapsto \sup \{\|a(J)\| \mid J \in W\}$ is a seminorm $p_{W}$ and $\inf p_{W}=\lim p_{W}$ where the limit is taken pointwise, since $p_{W}$ is a decreasing net.

Thus $p=\inf _{W} p_{W}$ is a seminorm. But $p(a)=0$ means $\lim _{W} \sup \{\|\hat{a}(J)\| J \in W\}=0$, i.e.

$$
\lim _{J \rightarrow I}\|\hat{a}(J)\|=0 .
$$

LEMMA 8.2. $a \in \tilde{I}$ iff $\lim _{J \rightarrow I}\|\hat{a}(J)\|=0$ (relative to the hull-kernel topology on $B$ ).

Proof. By definition of $\tilde{I}$ we have $a \in \tilde{I}$ iff for all $\varepsilon>0$ there is a hullkernel neighborhood $W$ such that $\|a+J\|<\varepsilon$ for $J \in W$. This holds iff $\lim _{J \rightarrow I}\|\hat{a}(J)\|=0$.

This shows that $a+\tilde{I} \mapsto|a+\tilde{I}|: R / \tilde{I} \rightarrow[0, \infty[$ is a well-defined norm. Since $p_{W}\left(a^{*} a\right)=p_{W}(a)^{2}$ we have $p\left(a^{*} a\right)=p(a)^{2}$.

It then follows almost immediately from known facts about $C^{*}$-algebras (e.g. $[23$, p. 16]) that it agrees with the quotient norm. We thus have $\|\tilde{a}(I)\|$ $=\|a+\tilde{I}\|=\lim \sup \{\|\hat{a}(J)\| \mid J \in W\}, I \in W$.

We now show that the field uniformity $\tilde{\mathfrak{U}}$ has a basis of sets $B_{\varepsilon}=\left\{\left(a_{1}\right.\right.$ $\left.\left.+\tilde{I}, a_{2}+\tilde{I}\right) \mid I \in B,\left\|a_{2}-a_{1}+\tilde{I}\right\|<\varepsilon\right\}$.

Indeed, $\tilde{\mathfrak{U}}$ has a basis of sets $\left(U_{\varepsilon}\right)_{1}^{B}=\left\{\left(a_{1}+\tilde{I}, a_{2}+\tilde{I}\right) \mid I \in B\right.$ and there is a neighborhood $W$ of $I$ in $B$ such that $J \in W$ implies $\left\|a_{2}-a_{1}+J\right\|<\varepsilon$ in $R / J\}$ by $\S 6$ (see 6.1 ).

If $(\tilde{I}, a+\tilde{I}) \in\left(U_{\varepsilon}\right)_{1}^{B}$, then $\|a+J\|<\varepsilon$ for all $J$ in some neighborhood $W$ of $I$.

Hence $\|a+\tilde{I}\|=\lim \sup _{V}\{\|a+J\| \mid J \in V, I \in V\} \leqq \varepsilon<2 \varepsilon$, so $\quad(\tilde{I}, a$ $+\tilde{I}) \in B_{2 \varepsilon}$. Thus $\left(U_{\varepsilon}\right)_{1}^{B} \subseteq B_{2 \varepsilon}$. Conversely, assume $(\tilde{I}, a+\tilde{I}) \in B_{\varepsilon}$, then $\|a+\tilde{I}\|<\varepsilon ;$ since $\|a+\tilde{I}\|=\lim _{V} \sup \{\|a+J\| \mid J \in V\}, I \in V$, then there 
must be a neighborhood $W$ of $I$ such that $\sup \{\|a+J\| \mid J \in W\}<\varepsilon$, whence $(\tilde{I}, a+\tilde{I}) \in\left(U_{\varepsilon}\right)_{1}^{B}$. Thus $B_{\varepsilon} \subseteq\left(U_{\varepsilon}\right)_{1}^{B}$.

Since the $B_{\varepsilon}$ form a basis of the field uniformity $\mathfrak{U}_{R}$ (see $\S 6$ ) we have

Proposition 8.3. With the quotient norm on $R / \tilde{I}$ we have $\|a+\tilde{I}\|$ $=\inf _{W} \sup _{J}\|a+J\|, J \in W, W$ ranging through the hull-kernel neighborhoods of $I$, and the field uniformity defined by this norm agrees with both the one derived from localization of $\hat{R}$ over $B$ and the canonical one associated with $R$ and $\{\tilde{I} \mid I \in B\}$. Thus the field star topology on $B^{\#}$ associated with $R$ and $\{\tilde{I} \mid I \in B\}$ is coarser than or equal to the hull-kernel topology. The function $J \mapsto\|a+J\|: B \rightarrow \boldsymbol{R}$ is upper semicontinuous in $I$ for all a iff $\tilde{I}=I$.

REMARK 1 . Note that we may let $W$ range through the collection of all $S(r)=\{J \in B \mid r \notin J\}, r \in R \backslash I$. Then $\|a+\tilde{I}\|=\inf _{r \in I} \sup _{r \notin J}\|a+J\|$.

REMARK 2. The function $J \mapsto\|a+J\|: B \rightarrow R$ is always lower semicontinuous; thus $\tilde{I}=I$ iff this function is continuous in $I$ for all $a \in R$. If $A$ is separable, then the set of points in which this is the case is a dense $G_{\delta}$ $[23$, p. 80].

If $R$ is separable, then Prim $R$ has a countable basis for its topology $\left[23\right.$, p. 64]. Thus $a \in \tilde{I}$ iff $\lim \left\|a+I_{n}\right\|=0$ for all sequences $I_{n}$ converging to $I$ in Prim $R$, provided $R$ is separable.

Our conclusion so far is that with a $C^{*}$-algebra $A$ and each subspace $B \subseteq \operatorname{Prim} A$ we can associate a uniform field $(\tilde{\pi}, \tilde{\mathfrak{U}}), \tilde{\pi}: \widetilde{E} \rightarrow B$ of $C^{*}$ algebras with stalks $\widetilde{E}_{I}=R / \tilde{I}, \tilde{I}=\{a \in R\|\lim \| a+J \|=0$ as $J$ approaches $I$ in $B\}$ such that the Gelfand morphism $R \rightarrow \Gamma^{b}(\pi)$ injects $R$ isomorphically into the $C^{*}$-algebra of bounded global sections. It has not been investigated to my knowledge whether or not $R \cong \Gamma^{b}(\pi)$ under the Gelfand representation if $R$ has an identity.

However, a coarser localization process was shown to yield the desired result. For the following assume that $R$ has an identity. Let $Z$ be the center of $R$. We then have the following

Proposition 8.4. The function $I \mapsto I \cap Z: \operatorname{Prim} R \rightarrow \operatorname{Spec} Z$ is a continuous surjection and is, in fact, the Stone-Cech compactification (Hausdorffization) (which is unique up to natural equivalence) [20, DAUNS and HOFMANN].

Let $\operatorname{Prim}^{\prime} R$ be the set Prim $R$ together with the coarsest topology making the map in 8.4 continuous. Then $\operatorname{Prim}^{\prime} R$ is quasi-completely regular in the sense explained before 7.9 (i.e. every point may be separated from a closed set not containing the point by a continuous function into $[0,1])$. In fact, if Prim $R \rightarrow X$ is a continuous function into a completely regular space it will remain continuous as a function Prim $R \rightarrow X$. We 
now proceed with the canonical localization process 6.6 relative to the topology on Prim' $R$. Specifically, for $I \in \operatorname{Prim} R$ we form the ideal $I^{\prime}=\bigcap_{U} \bigcup_{W} \bigcap_{J}(U+J), J \in W, W$ a neighborhood of $I$ in Prim' $R, U$ a zero neighborhood of $R$. In complete analogy to our previous discussion we obtain

Proposition 8.5. $I^{\prime}=\{a \in R\|\lim \| a+J \|=0$ as $J$ approaches $I$ in Prim' $^{\prime} R$ and $\left\|a+I^{\prime}\right\|=\inf _{W} \sup _{J}\|a+J\|, J \in W, W$ ranging through the neighborhoods of $I$ in Prim' $R$.

REMARK. It follows that $I^{\prime} \subseteq \tilde{I}$ for all $I \in \operatorname{Prim} R$; whence there is a morphism $R / I^{\prime} \rightarrow R / \tilde{I}$. Let $\left(\pi^{\prime}, \mathfrak{l}^{\prime}\right), \pi: E^{\prime} \rightarrow \operatorname{Prim}^{\prime} R$ be the canonical field of $C^{*}$-algebras associated with the family $\left\{I^{\prime} \mid I \in \operatorname{Prim} R\right\}$. It is isomorphic to the one obtained from the canonical field associated with the family Prim $R$ by localization relative to the topology of Prim' $R$, but also isomorphic to the one obtained from the canonical field associated with the family $\tilde{I}, I \in \operatorname{Prim} R$ by localization relative to the topology of Prim' $R$.

The Gelfand representation $R \rightarrow \Gamma\left(\pi^{\prime}\right)$ is injective since its kernel is $\bigcap\left\{I^{\prime} \mid I \in\right.$ Prim $\left.R\right\} \subseteq \bigcap$ Prim $R=\{0\}$. However, in this case we may apply Corollary 7.10 with $M=R^{\prime}$, since $R^{\prime} \leqq \Gamma\left(\pi^{\prime}\right)$ has approximate bounded partitions of unity as DAUNS and the author have shown [20,p. 96] (and this is not entirely trivial). We finally obtain

THEOREM 8.6 (The noncommutative Gelfand Naimark theorem). Let $R$ be a $C^{*}$-algebra with identity and let Prim' $R$ the space of its primitive ideals with the coarsest topology making the function $I \mapsto I \cap Z: \operatorname{Prim} R \rightarrow \operatorname{Spec} Z$ with the centrum $Z$ of $R$ continuous. Then there is a field of $C^{*}$-algebras over Prim' $R$ with stalks $R / I^{\prime}, I^{\prime}=\{a \in R\|\lim \| a+J \|=0$ as $J$ approaches $I$ in Prim' $\left.^{R}\right\}$ such that the Gelfand isomorphism of $R$ into the $C^{*}$-algebra of global sections in this field is an isomorphism.

It should be pointed out that an equivalent field representation can be given over the space Spec $Z$ in place of Prim' $R$ and this is in fact what was done by DaUns and HoFMANN. Indeed the field $\pi^{\prime}$ is constant over the sets $\{I \in \operatorname{Prim} R \mid I \cap Z=M\}, M \in \operatorname{Spec} Z=\operatorname{Max} Z$. Moreover, it can be shown that $I^{\prime}=\bigcap\{J \in \operatorname{Prim} R \mid J \cap Z=I \cap Z\}$; this is due to the fact that the function $M \rightarrow\|a+\bigcap\{J \in \operatorname{Prim} R \mid J \cap Z=M\}\|: \operatorname{Spec} Z \rightarrow R$ is upper semicontinuous $\left[20\right.$, p. 100]. Also, $I^{\prime}$ is the ideal generated in $R$ by $I \cap Z$. We thus obtain the following

COROLlary 8.7. If $R$ is a $C^{*}$-algebra with identity and $Z$ its center, then there is a uniform field of $C^{*}$-algebras over $\operatorname{Spec} Z$ such that the stalks are of the form $R / M^{\prime}$ with $M^{\prime}=\bigcap\{J \in \operatorname{Prim} R \mid J \cap Z=M\}, M \in \operatorname{Spec} Z$, and the Gelfand morphism maps $R$ isomorphically onto the $C^{*}$-algebra of global sections. Moreover, the field is the canonical field associated with the family 
of the $M^{\prime}, M \in \operatorname{Spec} Z$, and $M^{\prime}$ is the ideal generated in $R$ by $M_{X}$.

There are more special classes of $C^{*}$-algebras which allow particularly satisfactory representation theorems. Indeed, let $R$ be a $C^{*}$-algebra with identity and $\operatorname{Max} R$ its maximal ideal space. If $\operatorname{Max} R$ is Hausdorff (and thus Hausdorff compact), then the function $I \mapsto\|a+I\|: \operatorname{Max} R \rightarrow R$ is known to be continuous. This means that for Max $R$ as base space, the ideals $\tilde{I}$ equal $I$ for all $I \in \operatorname{Max} R$. Hence we obtain, by the methods outlined, a uniform field $(\pi, \mathfrak{U}), \pi: E \rightarrow$ Max $R$ of simple $C^{*}$-algebras with identity, such that the Gelfand representation is surjective. We thus have the following

COROLlaRY 8.8. Let $R$ be a $C^{*}$-algebra with identity and with Hausdorff maximal spectrum Max $R$. Then there is a field of simple $C^{*}$-algebras with identity over $\operatorname{Max} R$ with stalks $R / I, I \in \operatorname{Max} R$, such that the sequence

$$
0 \rightarrow \bigcap \operatorname{Max} R \rightarrow R \rightarrow \Gamma(\pi) \rightarrow 0
$$

is an exact sequence of $C^{*}$-algebras; in particular, $R / \bigcap \operatorname{Max} R \cong \Gamma(\pi)$.

This applies in particular to von Neumann algebras ( $W^{*}$-algebras) which are strongly semisimple and whose maximal ideal space is isomorphic to the maximal ideal space of the center under $I \mapsto I \cap Z$; we thus obtain the following result:

COROllary 8.9. Any $W^{*}$-algebra is isomorphic as a $C^{*}$-algebra to the $C^{*}$-algebra of all global sections in a field of simple $C^{*}$-algebras with identity over the space Max $R$ of maximal ideals of $R$; the stalk of the field over $I$ is $R / I$.

Note that $\operatorname{Max} R \cong \operatorname{Max} Z$ is a hyper-Stonean space. A $W^{*}$-algebra is strongly harmonic in the sense of $\mathrm{KOH}$ (see 1.28 and preceding discussion); thus there is a discrete version of the above representation theorem: The sheaf $\mathscr{R}=\mathscr{R}^{\prime}$ associated with $R$ according to $\S 1$ is a soft sheaf over a hyperStonean space, and its ring of global sections is isomorphic to the (discrete) ring underlying $R$. This has been exploited extensively by TELEMAN in his algebraic reduction theory of von Neumann algebras ([90], [91], and [92]).

It is possible to associate with each uniform field a sheaf over the same base space, namely, the sheaf of germs of local sections, and there is a bijection between the set of global sections in the field and the sheaf. If we start with a field of $C^{*}$-algebras, then we will obtain a sheaf of involutive algebras, and as involutive discrete algebras, the algebras of global sections are isomorphic of the field and the sheaf. However, this approach has not yet been systematically investigated; it certainly should be, since, at least for $C^{*}$-algebras one has a reasonably good field theory, and for arbitrary discrete rings, one has a better sheaf theory which, when specified to 
operator algebras such as $W^{*}$-algebras, has been shown by TeLEMAN to be of functional analytic interest. We should note, however, that the canonical sheaves associated with a $C^{*}$ - or $W^{*}$-algebra (as a discrete ring) carry less information as the associated fields.

In the case of the absence of an identity, appropriate modifications of these results are available; the image of the Gelfand representation, however, consists then of all global sections which are small outside members of a certain family of compact sets of Prim' $R$, respectively the complete regularization space of Prim $R$. The better process, however, in this case is to embed $R$ into a $C^{*}$-algebra with identity by taking the centroid $Z$ of $R$ and forming the split extension $R \times Z$ which is then reduced modulo the ideal $D=\{(a, z) \in R \times Z \mid a=-z\}$ where the center of $R$ is identified with those elements $\phi$ of the centroid $Z$ for which there is a central $z \in R$ with $\phi(a)=z a$ for all $a \in R$. (Recall that the centroid of $R$ is the set of all additive continuous linear maps $\phi: R \rightarrow R$ with $\phi(a b)=\phi(a) b=a \phi(b)$.) It can be shown that the $\widetilde{R}=(R \times Z) / D$ contains an isomorphic copy of $R$ as an ideal, has center isomorphic to $Z$, and that its primitive spectrum Prim $\tilde{R}$ is a quasi-compactification of Prim $R$ whose Hausdorffization $\operatorname{Spec} Z$ is the Čech compactification of Prim $R$. Over the latter we have a field according to 8.7 whose $C^{*}$-algebra of global sections is isomorphic to $\widetilde{R}$, and the image of $R$ is then given by all sections vanishing in a suitable fashion on the outside of the image of Prim $R$ in Spec Z. For the details see [20] and [21].

Fields of Hilbert spaces. Continuous fields of Hilbert spaces have been studied by Dixmier and Douady ([24], [26]); their definition deviates somewhat from ours. More recently, Takahashi resumed this topic [79] and used it, continuing earlier work by Kaplansky, to describe the structure of so-called Hilbert modules. A Hilbert module $H$ is a Banach space and a topological module over a $C^{*}$-algebra $R$ with identity together with a sesquilinear map $(\mid): H \times H \rightarrow R$ satisfying the axioms of an inner product on a Hilbert space, appropriately adjusted to the fact that it takes its values in $R$ instead of the complex field. The norm in $H$ is given by $|x|=\|(x \mid x)\|^{1 / 2}$. Note that every $C^{*}$-algebra is a Hilbert module over itself relative to the inner product $(a \mid b)=a b^{*}$. As an analytic analogue of some of the module representation theorems in $\S 1$ and $\S 5$ one obtains the following result:

THEOREM 8.10 (TAKAHASHI). Let $\mathrm{R}$ be a $\mathrm{C}^{*}$-algebra with identity and $\mathrm{H}$ a Hilbert-module over $R$. Let $Z$ be the center of $R$ and $(\rho, \mathfrak{B}), \rho: F \rightarrow \operatorname{Spec} Z$ the canonical field of $C^{*}$-algebras constructed in 8.7. Then there is a uniform field $(\pi, \mathfrak{U}), \pi: E \rightarrow \operatorname{Spec} Z$ of Hilbert modules over $R$ and a bilinear map of uniform fields $F \vee E \rightarrow \operatorname{Spec} Z$ making $\pi$ into a $\rho$-module (see 7.1) in such a way that each $E_{M}$ becomes an $F_{M}=R / M^{\prime}$-module (see 8.7 for a definition of $\left.M^{\prime}\right)$. The Gelfand morphisms $R \rightarrow \Gamma(\rho)$ and $H \rightarrow \Gamma(\pi)$ induce isomorphisms 
such that the diagram

$$
\begin{aligned}
R & \times H \longrightarrow H \\
& \downarrow \\
\Gamma(\rho) & \stackrel{\downarrow}{\times} \Gamma(\pi) \rightarrow \Gamma^{\downarrow}(\pi)
\end{aligned}
$$

commutes. If, in particular, $R$ is abelian, then $\pi$ is a uniform field of Hilbert spaces, and upon identifying $R$ with $C(\operatorname{Spec} R)$, the scalar multiplication of $R$ on $\Gamma(\pi)$ is given by $(f \cdot \sigma)(M)=f(M) \cdot \sigma(M)$.

In fact, TAKAHASHI shows in a very precise categorical fashion that the study of Hilbert modules over commutative $C^{*}$-algebras with identity is the same as the study of fields of Hilbert spaces over compact Hausdorff spaces. More accurately, consider the category Mod whose objects are Hilbert modules $M$ over commutative $C^{*}$-algebras $R$ with identity; since $R$ varies, we write $(R, M)$ for these objects. A morphism $(R, M) \rightarrow\left(R^{\prime}, M^{\prime}\right)$ is a pair $(f, \phi)$ consisting of an identity-preserving of $C^{*}$-morphism $f: R \rightarrow R^{\prime}$ and a Banach space morphism $\phi: M \rightarrow M^{\prime}$ such that $\phi(r \cdot m)=f(r) \cdot \phi(m)$ for $r \in R$ and $M \in M$. Then Mod will be called the category of Hilbert modules. Let Mod be the category whose objects are uniform fields of Hilbert spaces $\pi: E \rightarrow B$ over compact Hausdorff spaces $B$. A morphism from $\pi$ to a field $\rho: F \rightarrow C$ consists of a pair $(f, \phi)$, where $f: C \rightarrow B$ is continuous and $\phi$ is a continuous field map from the pullback field $E_{f} \rightarrow C$ of $\pi$ over $C$ into the field $\rho$; we thus have a commuting diagram

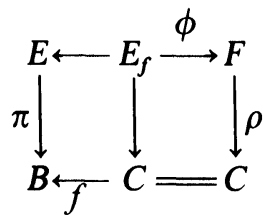

in which the left square is a pullback. The morphisms compose in a fashion known to the sheaf and category theoretician: $(f, \phi)\left(f^{\prime}, \phi^{\prime}\right)=\left(f^{\prime} f,\left(\phi_{f^{\prime}}\right) \phi^{\prime}\right)$, with $\phi_{f^{\prime}}$ defined via the pullback property in Figure 3 below

The category Mod is called the category of fields of Hilbert spaces.

THEOREM 8.11 (TAKAHASHI). There is a functor L:Mod $\rightarrow$ Mod which associates with each Hilbert module $(R, M)$ a field of Hilbert spaces over Spec $R$ and a functor $\Gamma:$ Mod $\rightarrow$ Mod which associates with each field $\pi: E \rightarrow B$ of Hilbert spaces the Hilbert module $(C(B), \Gamma(\pi))$ of global sections and the pair $(L, \Gamma)$ is an equivalence between Mod and Mod.

This is an analogue of a theorem in commutative discrete ring theory which says that the category of $R$-modules for commutative rings with identity is equivalent to the category of sheaves of commutative local rings (with certain restrictions); if one considers the subcategory $R$-Mod of $\operatorname{Mod}$ 


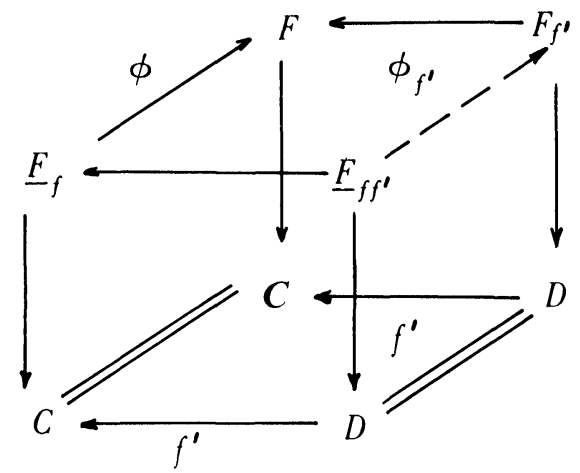

FIGURE 3

obtained by fixing $R$ and the subcategory $C(B)$-Mod of Mod obtained by fixing $B$, then one obtains an equivalence between $R$-Mod and $C(\operatorname{Spec} R)$ $M o d$ and we encounter here an analytical analogue of Mulvey's equivalences (see $5.23 \mathrm{ff}$.).

Topological f-algebras. We have seen that the representation of topological rings by global sections in fields is particularly manageable in the case of $C^{*}$-algebras. In [20] DAUNS and the author have introduced the class of $C$-algebras which satisfies enough conditions to make the whole theory work. Nevertheless, the field theory has not been very systematically tested for many other familiar categories of topological rings.

However, one class of rings which can be given a natural topology has been considered by DAUNS, namely, the class of topological $f$-rings whose sectional representation as discrete lattice ordered rings has been so carefully investigated by KeIMEL (see $\S 4$ ). The treatment of $f$-rings discussed by DAUNS in terms of field representation is, in some sense, a counterpart over the reals of the $C^{*}$-algebra case.

Firstly, we recall that, by 4.16 , the space $B=\operatorname{Max} \operatorname{Irr} R$ of maximal irreducible ideals is Hausdorff (and in fact is Hausdorff embedded into $\operatorname{Irr} A$ ). In any $l$-ring $R$ one writes $a^{+}=0 \vee a, a^{-}=0 \vee-a,|a|=a^{+}$ $+a^{-}$. We say that a topological ring with identity is a topological rational $f$-algebra if the sets $\{a \in A|| a \mid<(1 / n) \cdot 1\}, n=1,2,3, \ldots$, form a basis for the zero neighborhoods. This topology will also be called the natural one.

By a result of ISBELL, every $l$-ring can be embedded into a rational l-algebra. DAUns constructs the canonical uniform field of topological $f$-algebras $(\pi, \mathfrak{U}), \pi: E \rightarrow B$ associated with $R$ and $B=\operatorname{Max} \operatorname{Irr} R$ and shows that the field star topology on $B=\operatorname{Max} \operatorname{Irr} R$ agrees with the hullkernel topology $[18, \mathrm{p} .645]$. This means that the localization of $R \cong \Gamma(\pi)$ w.r. to the hull-kernel topology is not needed or, to put it in an equivalent fashion, that $\tilde{I}=I$ for all $I \in B$ where $\tilde{I}$ is constructed relative to this family of ideals. Thus we have a Gelfand representation $R \rightarrow \Gamma(\pi)$ of 
topological $f$-algebras with kernel Max $\operatorname{Irr} R$. Note that all stalks $R / I$ are totally ordered by 4.14. DAuns shows that the image of the Gelfand isomorphism $\phi$ is actually isomorphic to $R / \operatorname{ker} \phi$ as topological $f$-rings [18, pp. 640-641]. In order to see how large the image $R$ is in $\Gamma(\pi)$ we employ a partition of unity argument along KeIMEL's lines (see 4.7): If $\sigma$ is a nonnegative section in $\Gamma(\pi)$, and $\varepsilon$ is a positive rational number, we find for each point $I \in B=\operatorname{Max} \operatorname{Irr} R$ a neighborhood $U$ of $I$ and an element $a \in R$ such that $|\sigma(J)-\hat{a}(J)|<\varepsilon \cdot \hat{1}(J)$ for $J \in U$; we may replace $a$ by $a^{+}$without violating this estimate, hence we can assume that $0 \leqq a$. By the usual compactness argument we cover $B$ by neighborhoods $U_{1}, \ldots, U_{m}$ and find elements $a_{1}, \ldots, a_{n}$ such that $a_{k} \geqq 0$ and $\left|\sigma-\hat{a}_{k}\right|<\varepsilon \cdot 1$ on $U_{k}$. We form the ideals $I_{k}=\bigcap\left(B \backslash U_{k}\right), k=1, \ldots, n$, and conclude $R=I_{1}+\cdots+I_{n}$ as usual. The element $b=a_{1} \vee \cdots \vee a_{n}$, as any element in $I_{1}+\cdots+I_{n}$ may then be represented in the form $b=b_{1} \vee \cdots \vee b_{n}$ with $0 \leqq b_{k} \in I_{k}$, $k=1, \ldots, n$. We let $a=\left(a_{1} \wedge b_{1}\right) \vee \cdots \vee\left(a_{n} \wedge b_{n}\right)$. We now take an arbitrary index $j$ with $1 \leqq j \leqq n$, and pick an arbitrary $I \in U_{j}$.

As an intermediate lemma we observe that in an $l$-group the relation $|u-v|<e$ implies $|(u \wedge w)-(v \wedge w)|<e$ for $0 \leqq u, v, w$ : Indeed $|u-v|$ $<e$ is equivalent to $u-v<e$ and $v-u<e$, i.e. to $u-e<v$ and $v-e$ $<u$. Then $(u \wedge w)-e=(u-e) \wedge(w-e)<(u-e) \wedge w<v \wedge w$, and similarly $(v \wedge w)-e<u \wedge w$. These two inequalities imply the assertion $|(u \wedge w)-(v \wedge w)|<e$.

Thus, continuing our argument, we may conclude that for any $k$ with $I \in U_{k}$ we have

(i) $\left|\left(\hat{a}_{k} \wedge \hat{b}_{k}\right)-\left(\hat{a}_{j} \wedge \hat{b}_{k}\right)\right|(I)<2 \varepsilon \cdot \hat{1}(I)$.

If $k$ is such that $I \notin U_{k}$, then $b_{k} \in I$, and thus $\left(\hat{a}_{k} \wedge \hat{b}_{k}\right)(I)=\left(\hat{a}_{j} \wedge \hat{b}_{k}\right)(I)$ $=\hat{0}(I)$. Thus (i) holds in fact for all $k=1, \ldots, n$. By the distributivity of $R$ as a lattice we have $a_{j}=a_{j} \wedge b=a_{j} \wedge \vee_{k} b_{k}=\vee_{k}\left(a_{j} \wedge b_{k}\right)$. We now compute $\quad \hat{a}_{j}(I)=\vee_{k}\left(\hat{a}_{j} \wedge \hat{b}_{k}\right)(I)<\vee_{k}\left(a_{k} \wedge b_{k}\right)(I)+2 \varepsilon \cdot \hat{1}(I)=\hat{a}(I)+2 \varepsilon \cdot \hat{1}(I)$ and similarly $\hat{a}(I)<\hat{a}_{j}(I)+2 \varepsilon \cdot \hat{1}(I)$.

Hence

(ii) $\left|\hat{a}(I)-\hat{a}_{j}(I)\right|<2 \varepsilon \cdot \hat{1}(I)$ for $I \in U_{j}$. Thus, by the choice of the $a_{j}$, for $I \in U_{j}$ we have $|\hat{a}(I)-\sigma(I)| \leqq\left|\hat{a}(I)-\hat{a}_{j}(I)\right|+\left|\hat{a}_{j}(I)-\sigma(I)\right|<2 \varepsilon \cdot \hat{1}(I)$ $+\varepsilon \cdot \hat{1}(I)=3 \varepsilon \cdot \hat{1}(I)$. Hence $|\hat{a}-\sigma| \leqq 3 \varepsilon \cdot \hat{1}$.

This shows that indeed the image $\hat{R}$ of $R$ under the Gelfand morphism is dense in $\Gamma(\pi)$. If, in particular, $R$ happens to be complete in its topology then, due to the fact that $R$ is isomorphic to $R / \cap B$, we may conclude that $\hat{R}=\Gamma(\pi)$.

We therefore arrived at the following result which is a slight improvement of a theorem of DAUNS.

THEOREM 8.12. Let $R$ be a topological rational f-algebra with identity (endowed with its natural topology). Then there is a uniform field $(\pi, \mathfrak{u})$, 
$\pi: E \rightarrow \operatorname{Max} \operatorname{Irr} R$ of totally ordered f-algebras with their natural topology with stalks $E_{I}=R / I$ over the compact Hausdorff space of all maximal irreducible ideals such that the Gelfand representation (i) maps $R$ into $\Gamma(\pi)$ with kernel $\bigcap \operatorname{Max} \operatorname{Irr} R$, (ii) is a quotient map onto its image, and (iii) has dense image in $\Gamma(\pi)$; in particular, if $R$ is complete relative to its natural topology, then $R / \bigcap \operatorname{Max} \operatorname{Irr} R \cong \Gamma(\pi)$ as topological f-rings. In this case $R$ is a $C(\operatorname{Max} \operatorname{Irr} R, R)$-module in an obvious fashion.

This is presumably not the best theorem in this direction yet; if one associates with $R$ the canonical field over $\operatorname{Irr} R$ with the stalks $R / I$ as constructed in 6.7, then the Gelfand morphism associated with this fiela is in fact an injection of $R$ into the ring of its global sections since $\bigcap \operatorname{Irr} R=(0)$ by 4.4. Is it surjective? After 4.16 and remarks following 1.27 there is a continuous retraction $\mu: \operatorname{Irr} R \rightarrow \operatorname{Max} \operatorname{Irr} R$ which associates with each $I$ a unique maximal irreducible ideal $\mu(I)$ containing $I$, and $\mu$ is, in fact the Hausdorffization map. If one considers the field which one obtains from localization according to 6.6 relative to the coarser topology on $\operatorname{Irr} R$ defined as the coarsest making $\mu$ continuous, one seems to be in a situation analogous to the one for $C^{*}$-algebras which resulted in the general Gelfand Naimark Theorems 8.6 and 8.7. It is not unreasonable to expect that these results carry over to the case of topological $f$-rings, perhaps to some extent even to topological $l$-rings.

It is, nevertheless, instructive to compare the field representation theorem of a topological, strongly semisimple topological rational $f$ algebra in 8.11 with the corresponding sheaf representation over Max $\operatorname{Irr} R$ according to 4.8. By a remark following 4.16 the sheaf representation yields an isomorphism of the discrete $f$-algebra $R$ onto the $f$-algebra of global sections in a sheaf of local $f$-algebras over the compact Hausdorff space Max Irr $R$. In the field representation the advantage is two-fold: Firstly, the stalks are totally ordered simple $f$-algebras; secondly, the topological structure of the given algebra is fully represented. Just as in the case of $C^{*}$-algebras, the best-known example illustrating the situation is an algebra of the form $R=C(X, R)$ for a compact Hausdorff space $X$ (the complex version illustrating the $C^{*}$-algebra situation).

\section{INDEX}

References in the index are to section numbers whenever a term is introduced in a specified section; otherwise it is indicated that a term is introduced preceding or following a numbered section by " $<$ ", resp., " $>$ ". Example: " $l$-ideal $>4.1$ " means that the term $l$-ideal occurs for the first time in the discussion following $\$ 4.1$.

approximate bounded partition of unity (identity)
Baer extension

7.6 Baer rings
$>3.10$ $<3.7$ 


\begin{tabular}{|c|c|c|c|}
\hline biregular & $>1.31,3.1$ & patch topology & $>3.8$ \\
\hline Boolean decomposition & 3.3 & Pierce decomposition; Pierce sheaf & 3.4 \\
\hline bounded; locally bounded & $>7.2$ & presheaf & 1.1 \\
\hline bounded section & $<5.19$ & quasi-compact & $<1.9$ \\
\hline canonical field & 5.15 & quasi-completely regular & $<7.9$ \\
\hline centroid & $3.1,>8.9$ & quasi-support & $>1.23$ \\
\hline compatible (with a bilinear map) & $>7.2$ & $\operatorname{Rad} E$ : radical (of a standard sheaf) & $<1.8$ \\
\hline dimension & $<7.7$ & regular (maximal ideals) & $>1.27$ \\
\hline field by localization & 6.5 & relative identity & $>1.10$ \\
\hline fields of Hilbert spaces & $8.10,8.11$ & ringed space & 1.2 \\
\hline field *-topology; field star topology & $y>5.14$ & section & 5.2 \\
\hline field uniform structure & $>5.1$ & semiconnected & 5.8 \\
\hline Gelfand morphism (representation) & $>1.7$ & serration & 5.2 \\
\hline & $0,<5.20$ & sheaf & 1.1 \\
\hline Grothendieck sheaf & $<2.1$ & SIN-group & $>5.14$ \\
\hline harm & $<1.28$ & $\phi$-soft & $1.11,4.8$ \\
\hline Hausdorff embedding & 1.26 & smooth & 5.10 \\
\hline hereditary (etc.) & $<3.5$ & stalk & 1.1 \\
\hline Hilbert modules & $<8.10$ & Stone-Cech compactification $>1.27$ & $27,>3.1$ \\
\hline homogeneous uniform field & 5.12 & & $>8.9$ \\
\hline hull-kernel topology & $<1.9$ & nonic ring & $<1.28$ \\
\hline irreducible $l$-ideal & 4.3 & stru & 5.12 \\
\hline Keimel's decomposition, sheaf & 4.12 & t system & $.5,<4.7$ \\
\hline$l$-ideal & $>4.1$ & $U$-close & $>6.1$ \\
\hline l-ring & 4.1 & uniform field & $<5.4$ \\
\hline locally convex field & 7.8 & uniform field of rings & 7.1 \\
\hline local ring & $<1.26$ & ly homogeneous & 5.12 \\
\hline local $l$-ring & $<4.10$ & von Neumann algebras & $>3.2$ \\
\hline locally bounded & $>7.2$ & von Neumann regular & $1.31,3.9$ \\
\hline locally zero (section) & $>1.5$ & weakly biregular & $<3.2$ \\
\hline partition of identity & $<1.10$ & zero dimensional & $>1.29$ \\
\hline
\end{tabular}

\section{LIST OF SYMBOLS}

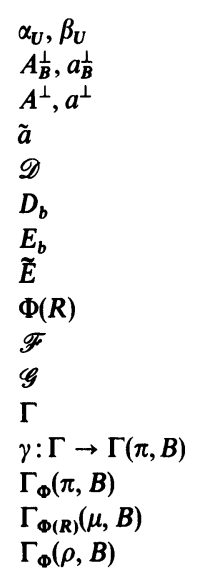

$\begin{array}{rl}>5.7 & \tilde{G}_{c} \\ >1.14,>4.6 & \text { Irr } R \\ <1.22 & \mathscr{H} \\ 1.4 & \tilde{K}_{b}, \tilde{K}(U) \\ <3.9 & I, m \\ 1.1 & K_{b}, K(U) \\ 1.2 & \lambda, \lambda_{b U} \\ >6.1 & \mathscr{M} \\ 1.11 & \mu \\ <3.9 & \mathcal{O}(B) \\ <2.1 & ? \text { po } \\ 1.1 & \mathscr{P} \\ 1.3 & \Phi \\ 1.11 & \tilde{\pi} \\ 1.12 & (\pi, \mathfrak{l n}) \\ 4.7 & \operatorname{Rad} E\end{array}$


$R$-Mod, $\mathscr{R}$-Mod

$\operatorname{Spec} R$

$S(a)$

$S(A)$

$(\mathrm{X}, \mathrm{e})$

$$
\begin{array}{rl}
>5.22 & \mathfrak{U},[\mathfrak{U}] \\
<1.9 & \tilde{\mathfrak{U}}, U^{C}, U_{1}^{C} \\
>1.9 & \mathfrak{U}_{G} \\
>1.14 & U^{\prime} \\
>1.10 &
\end{array}
$$

\section{BiBLIOGRAPHY}

(Entries numbered 16a. etc. were added in proof.)

1. D. H. Adams, Semigroups with no non-zero nilpotent elements, Math. Z. 123 (1971), 168-176.

2. R. F. Arens and I. Kaplansky, Topological representation of algebras, Trans. Amer. Math. Soc. 63 (1948), 457-481. MR 10, 7.

3. B. Banaschewski, Maximal rings of quotients of semi-simple commutative rings, Arch. Math. (Basel) 16 (1965), 414-420. MR 33 \# 7363.

4. G. M. Bergman, Hereditary commutative rings and centers of hereditary rings, Proc.

London Math. Soc. 23 (1971), 214-236.

5. - Boolean rings of projection maps (to appear).

6. - Hereditarily and cohereditarily projective modules (to appear).

7. - Notes on epimorphisms of rings, Seminar Notes.

8. R. Bkouche, Idéaux mous d'un anneau commutatif. Applications aux anneaux de fonctions, C. R. Acad. Sci. Paris 260 (1965), 6496-6498. MR 31 \# 1268.

9. - Pureté, mollesse et paracompacité, C. R. Acad. Sci. Paris 270 (1970), 1653-1655.

10. Couples spectraux et faisceaux associés. Applications aux anneaux de fonctions,

Bull. Soc. Math. France 98 (1970), 253-295.

11. N. Bourbaki, Éléments de mathématique. Fasc. XXVII. Algèbre commutative, Actualités Sci. Indust., no. 1290, Hermann, Paris, 1961. MR 36 \# 146.

12. A. Brezuleanu and $R$. Diaconescu, Sur la duale de la catégorie des treillis, Rev. Roumaine Math. Pures Appl. 14 (1969), 311-323. MR 41 \# 116.

13. A. Brezuleanu, Sur les schémas de treillis, Rev. Roumaine Math. Pures Appl. 14 (1969), 949-954.

14. P. M. Cohn, Skew fields of fractions, and the prime spectrum of a general ring, Lecture Notes in Math. 246, Springer-Verlag, Berlin and New York, 1971.

15. S. D. Comer, $A$ sheaf theoretical duality theory for cylindrical algebras, Trans. Amer. Math. Soc. (to appear).

16. (1971), 29-38.

16a. - Elementary properties of structures of sections (to appear).

17. J. Dauns, Representation of f-rings, Bull. Amer. Math. Soc. 74 (1968), 249-252. MR 36 \# 3699.

18. MR 41 \# 130.

19. J. Dauns and K. H. Hofmann, The representation of biregular rings by sheaves, Math. Z. 91 (1966), 103-123. MR 32 \#4151.

20. 180 pp. MR 40 \# 752.

21. - Spectral theory of algebras and adjunction of identity, Math. Ann. 179 (1969), 175-202. MR 40 \# 734.

22. G. Davis, Rings with orthogonality relations, Bull. Austral. Math. Soc. 4 (1971), 163-178.

22a. - Representation and extension of semi-prime rings (to appear).

23. J. Dixmier, Les $C^{*}$-algèbres et leurs représentation, 2ième éd., Cahiers Scientifiques, fasc. 29, Gauthier-Villars, Paris, 1969. MR 39 \#442.

24. , Champs continus d'espaces hilbertiens et de $C^{*}$-algèbres. II, J. Math. Pures Appl. (9) 42 (1963), 1-20. MR 27 \#603.

25. ㄴ, Ideal center of a $C^{*}$-algebra, Duke Math. J. 35(1968), 375-382. MR 37 \# 5703.

26. J. Dixmier and A. Douady, Champs continus d'espaces hilbertiens et de $C^{*}$-algèbres, Bull. Soc. Math. France 91 (1963), 227-284. MR 29 \# 485.

26a. D. P. Ellerman, Sheaves of relation structures and ultraproducts, Boston Univ. Research reports $71-19$ (1971), vi +83 pp.

27. H. Evans, Various topics concerning Baer rings, Dissertation, Tulane University, New Orleans, La., 1972. 
28. J. M. G. Fell, The structure of algebras of operator fields, Acta Math. 106 (1961), 233-280. MR 29 \#1547.

29. - Algebras and fiber bundles, Pacific J. Math. 16(1966), 497-503. MR 33 \# 2674.

30. - An extension of Mackey's method to algebraic bundles over finite groups, Amer. J. Math. 91 (1969), 203-238. MR 40 \# 735.

31. , An extension of Mackey's method to Banach*-algebraic bundles, Mem. Amer. Math. Soc. No. 90 (1969), 168 pp. MR 41 \# 4255.

32. B. R. Gelbaum, Banach algebra bundles, Pacific J. Math. 28 (1969), 337-349. MR 39 \#6077.

33. R. Giles, Foundations for quantum mechanics, Queen's Mathematical Preprint \#26, Kingston, Ontario, 1969, 85 pp.

34. L. Gillman, Rings with Hausdorff structure space, Fund. Math. 45 (1957), 1-16. MR 19, 1156.

35. J. G. Glimm, A Stone-Weierstrass theorem for $C^{*}$-algebras, Ann. of Math. (2) 72 (1960), 216-244. MR 22 \# 7005.

36. $R$. Godement, Sur la théorie des représentations unitaires, Ann. of Math. (2) 53 (1951), 68-124. MR 12, 421.

37. A. Grothendieck and J. Dieudonné, Éléments de géométrie algébrique. I. Le langage des schémas, Inst. Hautes Études Sci. Publ. Math. No. 4 (1960), 228 pp. MR 36 \# $177 \mathrm{a}$.

37a. M. Henriksen, On the space of minimal prime ideals. II, Notices Amer. Math. Soc. 19 (1972), A-62. Abstract 691-13-11.

38. M. Henriksen and M. Jerison, The space of miminal prime ideals of a commutative ring, Trans. Amer. Math. Soc. 115 (1965), 110-130. MR 33 \# 3086.

39. M. Hochster, Prime ideal structure in commutative rings, Trans. Amer. Math. Soc. 142 (1969), 43-60. MR 40 \# 4257.

40. ㄴ Totally integrally closed rings and extremal spaces, Pacific J. Math. 32 (1970), 767-779. MR 41 \#1718.

41. - Noncommutative algebraic geometry. I, Seminar Notes and Correspondence with G. M. Bergman.

41a. - The minimal prime spectrum of a commutative ring, Canad. J. Math. 23(1971), 749-758.

42. K. H. Hofmann, Gelfand-Naimark theorem for non-commutative topological rings,

Proc. Second Sympos. General Topology Appl. (Prague, 1966), Prague, 1967, pp. 184-189.

43. - Extending $C^{*}$-algebras by adjoining an identity, Contributions to Extension

Theory of Topological Structures, Proc. Sympos. (Berlin, 1967), Deutscher Verlag Wissenschaften, Berlin, 1969, pp. 119-125.

44. - Representations of algebras by continuous sections, Audio Recordings of Math. Lectures, no. 32, Amer. Math. Soc., Providence, R.I., 1971.

45. K. H. Hofmann and K. Keimel, A general character theory for partially ordered sets and lattices, Mem. Amer. Math. Soc. (to appear).

46. I. Kaplansky, The structure of certain operator algebras, Trans. Amer. Math. Soc. 70 (1951), 219-255. MR 13, 48.

47. K. Keimel, Représentation d'anneaux réticulés dans des faisceaux, C. R. Acad. Sci.

Paris Sér. A-B 266 (1968), A124-A127. MR 37 \#6224.

48. Sér. A-B 266 (1968), A524-A525. MR 37 \#6225.

49. — Darstellung von Halbgruppen und universellen Algebren durch Schnitte in Garben; bireguläre Halbgruppen, Math. Nachr. 45 (1970), 81-96.

50. - Représentation d'anneaux et de groupes réticulés par des section dans des faisceaux, Thèse, Paris, 1970.

51. Math. 22 (1970), 1071-1078.

52. (1971), 55-63.

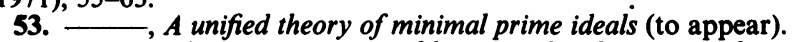

54. - The representation of lattice-ordered groups and rings by sections in sheaves, Lecture Notes in Math. 248, Springer-Verlag, Berlin and New York, 1971.

55. J. Kist, Compact spaces of minimal prime ideals, Math. Z. 111 (1969), 151-158. MR 39 \#6872.

56. - Representing rings by sections; complexes, J. Austral. Math. Soc. (to appear). 57. K. Koh, On functional representations of a ring without nilpotent elements, Canad. Math. Bull. 14 (1971), 349-352. 
58. - On a representation of a strongly harmonic ring by sheaves, Proc. Amer. Math. Soc. (to appear).

59. K. Koh and J. Luh, Maximal regular right ideal space of a primitive ring (to appear).

60. J. Lambek, Lectures on rings and modules, Blaisdell, Waltham, Mass., 1966. MR 34 \# 5857.

61. On the representation of modules by sheaves of factor modules (to appear).

62. I. G. Macdonald, Algebraic geometry. Introduction to schemes, Benjamin, New York, 1968. MR 39 \# 205.

62a. J. Mack, Fields of topological spaces (to appear).

63. A. Mallios, On topological algebra sheaves of nuclear type, Studia Math. 28 (1970), 215-220.

64. A. C. Mewborn, Some conditions on commutative semiprime rings, J. Algebra 13(1969), 422-431.

65. - Regular rings and Baer rings (to appear).

66. G. O. Michler and O. E. Villamayor, On rings whose simple modules are injective,

J. Algebra (to appear).

67. C. J. Mulvey, On ringed spaces, Dissertation, University of Sussex, 1970.

68. - Représentation des produits sous-directs d'anneaux par espaces annelés, $\mathrm{C} . \mathrm{R}$. Acad. Sci. Paris Sér. A-B 270 (1970), A564-A567. MR 41 \# 1808.

69. $-A$ condition for a ringed space to be a generator in its category of modules, J. Algebra 15 (1970), 312-313. MR 41 \# 3567.

70. M. A. Naìmark, On a continuous analogue of Schur's lemma, Dokl. Akad. Nauk SSSR 98 (1954), 185-188. (Russian) MR 16, 597.

71. David E. Peercy, Complexes and the complete Baer extension of a commutative ring (to appear).

72. R. S. Pierce, Modules over commutative regular rings, Mem. Amer. Math. Soc. No. 70 (1967), 112 pp. MR 36 \# 151.

73. Y. Quentel, Sur la compacité du spectre minimal d'un anneau, Bull. Soc. Math. France 99 (1971), 265-275.

74. J.-E. Roos, Locally distributive spectral categories and strongly regular rings, Reports of the Midwest Category Seminar, Springer, Berlin, 1967, pp. 156-181. MR 37 \# 279.

75. S. A. Selesnick, Lattice schemes and maps of pretopological spaces (to appear).

76. T. P. Speed, $A$ note on commutative Baer rings (to appear).

77. G. Spirason and E. Strelecki, $A$ note on $P_{t}$-ideals (to appear).

78. R. G. Swan, Vector bundles and projective modules, Trans. Amer. Math. Soc. 105(1962), 264-277. MR 26 \#785.

79. A. Takahashi, Fields of Hilbert modules, Dissertation, Tulane University, New Orleans, La., 1971.

80. S. Teleman, Analyse harmonique dans les algèbres regulières, Rev. Roumaine Math. Pures Appl. 13 (1968), 691-750.

81. L La représentation des anneaux tauberiens discrets par des faisceaux, Rev. Roumaine Math. Pures Appl. 14 (1969), 249-264. MR 40 \#737a.

82. —_ La représentation des anneaux réguliers par les faisceaux, Rev. Roumaine Math. Pures Appl. 14 (1969), 703-717. MR 40 \# 737b.

83., Représentation par faisceaux des modules sur les anneaux harmoniques, $\mathrm{C} . \mathrm{R}$. Acad. Sci. Paris Sér. A-B 269 (1969), A753-A756. MR 41 \#276.

84. - Représentation par faisceaux des modules sur des algèbres harmoniques, Rev. Roumaine Math. Pures Appl. 16 (1971), (to appear).

85. _ La représentation des algèbres de von Neumann finies par faisceaux, Rev. Roumaine Math. Pures Appl. 15 (1970), 143-151.

86. - Sur les anneaux réguliers, Rev. Roumaine Math. Pures Appl. 15 (1970), 407-434. MR 41 \#8472.

87. - On the regular rings of John von Neumann, Rev. Roumaine Math. Pures Appl. 15 (1970), 735-742.

88., Théorème de de Rham pour les algèbres harmoniques, $\mathrm{C}$. R. Acad. Sci. Paris Sér. A-B 269 (1969), A1119-A1121. MR 41 \#3570.

89. - The theorem of de Rham for harmonic algebras (to appear)

90. - Representation on von Neumann algebras by sheaves, Acta Sci. Math. (Szeged) (to appear).

91. - Algebraic reduction of von Neumann algebras (to appear).

92. - Theory of harmonic algebras with applications to von Neumann algebras and cohomology and locally compact spaces (de Rham's theorem), Lecture Notes in Math 248, Springer-Verlag, Berlin and New York, 1971. 
93. J. Tomiyama and M. Takesaki, Applications of fiber bundles to the certain class of $C^{*}$-algebras, Tôhoku Math. J. (2) 13 (1961), 498-522. MR 25 \# 2465.

94. J. Tomiyama, Topological representation of $C^{*}$-algebras, Tôhoku Math. J. (2) 14 (1962), 187-204. MR 26 \# 619.

94a. J. Varela, The automorphism group of fields of $C^{*}$-algebras, Dissertation, Tulane University, New Orleans, La., 1972.

95. J. Vrabec, Adjoining a unit to a biregular ring, Math. Ann. 188 (1970), 219-226.

96. R. Wiegand, Modules over universal regular rings, Pacific J. Math. 39 (1971).

97. —_, Regular preschemes (to appear).

98. _ Globalization theorems for locally finitely generated modules, Pacific J. Math. 38 (1971).

98a. - Generators of modules over commutative rings (to appear). 